\title{
Peroxiredoxins in Plants and Cyanobacteria
}

\author{
Karl-Josef Dietz
}

\begin{abstract}
Peroxiredoxins (Prx) are central elements of the antioxidant defense system and the dithiol-disulfide redox regulatory network of the plant and cyanobacterial cell. They employ a thiol-based catalytic mechanism to reduce $\mathrm{H}_{2} \mathrm{O}_{2}$, alkylhydroperoxide, and peroxinitrite. In plants and cyanobacteria, there exist 2-CysPrx, 1-CysPrx, PrxQ, and type II Prx. Higher plants typically contain at least one plastid 2-CysPrx, one nucleo-cytoplasmic 1CysPrx, one chloroplast PrxQ, and one each of cytosolic, mitochondrial, and plastidic type II Prx. Cyanobacteria express variable sets of three or more Prxs. The catalytic cycle consists of three steps: (i) peroxidative reduction, (ii) resolving step, and (iii) regeneration using diverse electron donors such as thioredoxins, glutaredoxins, cyclophilins, glutathione, and ascorbic acid. Prx proteins undergo major conformational changes in dependence of their redox state. Thus, they not only modulate cellular reactive oxygen species- and reactive nitrogen speciesdependent signaling, but depending on the Prx type they sense the redox state, transmit redox information to binding partners, and function as chaperone. They serve in context of photosynthesis and respiration, but also in metabolism and development of all tissues, for example, in nodules as well as during seed and fruit development. The article surveys the current literature and attempts a mostly comprehensive coverage of present day knowledge and concepts on Prx mechanism, regulation, and function and thus on the whole Prx systems in plants. Antioxid. Redox Signal. 15, 1129-1159.
\end{abstract}

I. Introduction 1130

II. Classification of Plant Prxs and Their Distribution in Plants and Cyanobacteria 1130

III. Peroxidase Activity of Peroxiredoxins 1132

IV. Conformational Dynamics and Interacting Partners of Prx In Vitro and In Vivo 1134

V. Typical 2-Cys Peroxiredoxins 1135

A. Characteristics of 2-CysPrxs

B. Reduction of oxidized 2-CysPrx 1136

C. Consequences of 2-CysPrx deficiency 1137

VI. Peroxiredoxin Q, an Atypical 2-CysPrx of Chloroplasts and Cyanobacteria 1138

VII. 1-Cysteine Peroxiredoxin 1140

VIII. Type II Peroxiredoxins, the Most Widely Distributed Atypical 2-CysPrxs in Plants 1140

A. Principle features of type II Prx 1140

B. Cytosolic PrxII 1142

C. Plastid PrxIIE 1142

D. Mitochondrial PrxIIF 1142

E. Cyanobacterial type II Prx 1143

IX. Plant Glutathione Peroxidases 1143

X. Posttranslational Regulation of Peroxiredoxin Activities 1143

A. Hyperoxidation and sulfiredoxin 1143

B. Nitrosylation 1144

C. Other posttranslational Prx modifications 1145

XI. Role of Peroxiredoxins in Plant Metabolism 1145

A. Plastids and photosynthesis 1146

B. Mitochondria and respiration 1147

Reviewing Editors: Bob Buchanan, Leopold Flohé, Christine Foyer, Juan-José Lázaro, Ken Motohashi, Luis Netto, Natalia Ozolina, and Ricardo A. Wolosiuk

Department of Biochemistry and Physiology of Plants, Bielefeld University, Bielefeld, Germany. 
XII. Peroxiredoxins as Chaperone and in Plant Redox Signaling

A. Prx as chaperone

B. Prx in ROS-dependent signaling

C. Prx in RNS-dependent signaling

A. Basal pattern of Prx expression and developmental control

B. Regulation of plastid Prx expression by retrograde signaling

C. Stress-dependent regulation

\section{Introduction}

$\mathbf{P}$ LANT PROTEIN LySATES typically contain about $2 \%$ cysteine. For a long time it was assumed that Cys in proteins from plasmatic compartments mostly adopt the reduced thiol state, whereas Cys of extraplasmatic proteins are preferentially oxidized to the disulfide form for tertiary structure stabilization. Beginning with the finding of thiol-dependent Calvin cycle enzyme regulation in photosynthesis (158) and accelerated by high-throughput technologies with the advent of proteomics and mass spectrometric identification of proteins (171) it became clear that thiols undergo various posttranslational modifications with significance for protein function. Thus, thiol-disulfide transitions of proteins frequently occur in catalysis, regulation, and signaling. This also implies that cells must have evolved mechanisms to reverse protein disulfides to dithiols and to protect thiols from nonspecific oxidation by reactive oxygen species (ROS) and reactive nitrogen species (RNS). In addition to the disulfide form, Cys thiols may be oxidized to sulfenic, sulfinic, and sulfonic acid derivatives or to nitrosothiol (32). In contrast to thiol-disulfide transitions, thiol oxidation to oxygenated derivatives is mostly an uncontrolled reaction of thiols with ROS, which is encountered as consequence of a strongly oxidative environment in the cell. In some cases, sulfenic acid formation of thiols in enzymes may be part of the catalytic cycle, for example, in peroxiredoxins (Prxs), which efficiently detoxify a broad set of peroxide substrates. Thus, Prxs act as thiol-dependent peroxidases using a thiol redox mechanism and have the primary function as high-affinity peroxide traps protecting other targets such as protein thiols from oxidation. After the transient generation of the sulfenic acid intermediate, the next reaction step involves thiol-disulfide transition of the catalytic Cys-OH residue with either another Cys of the same or an adjacent subunit, or with another thiol-containing compound. Thus, the catalytic cycle consists of three steps: (i) peroxide reduction, (ii) resolving step, and (iii) regeneration step (Fig. 1A), which will be discussed below. In addition to their peroxide detoxification activity, Prx undergo redoxdependent conformational switches and interact in a conformation-dependent manner with various proteins. Prxs are present in all kingdoms of life and almost all organisms. Only few exceptions have been found from this rule: thus, the sequenced genomes of the microbes Mesoplasma florum (Mesoplasma Florum Sequencing Project/Broad Institute: www.broad.mit.edu) and Borrelia burgdorferi (52) lack genes encoding Prxs.

Higher plant Prxs were first described in 1996, when the Hv-1-CysPrx (166) and the Hv-2-CysPrx (6) were reported from barley (Hordeum vulgare) and subsequently for many other species with oxygenic photosynthesis, including the cyanobacterium Synechocystis Pasteur culture collection (PCC)6803 (82), the liverwort Riccia fluitans (60), spinach (7), winter rye (16), and Arabidopsis thaliana (8). The third Prx type was isolated and described by Verdoucq et al. (178) and Rouhier et al. in ref. (151). Finally, the plant homolog of prokaryotic bacterioferritin comigratory protein then named PrxQ was identified in Sedum lineare (85). Each of these Prx types has been analyzed in quite some detail. The studies have proven their important roles in antioxidant defense and redox signaling during plant development and environmental acclimation. The following review surveys the current literature and attempts a mostly comprehensive coverage of present day knowledge and concepts on Prx mechanism, regulation, and function and thus on the whole Prx systems in plants. Recent research has assigned conformation- and redoxdependent functions to specific Prxs in plants that include site-specific peroxidase activity, role as redox sensor and chaperone, and as binding partner to target proteins.

\section{Classification of Plant Prxs and Their Distribution in Plants and Cyanobacteria}

The Prx family can be divided in six groups, named A, B, C, $\mathrm{D}, \mathrm{E}$, and F, based on sequence and structure similarities and positions of conserved cysteinyl residues (57, 123, 124). Group E comprises bacterial peroxidases and group F Prx homologs in archaea. Prx from the groups A to D are common and conserved in higher plants, for example, the genome of $A$. thaliana encodes genes for each of the four types of $\operatorname{Prx}(61,62)$ (Fig. 1B). According to the more commonly used nomenclature, the A-type Prx corresponds to the (typical) 2-Cys peroxiredoxin (2-CysPrx), the B-type Prx to the (typical) 1-Cys peroxiredoxin (1-CysPrx), the C-type Prx to peroxiredoxin Q (PrxQ), and the D-type Prx to type II peroxiredoxins (PrxII) (123). PrxQ and PrxII also are termed atypical 2-CysPrx (57). All the four Prx sub-classes are also present in the genomes of Populus trichocarpa, Oryza sativa, and the cyanobacterium Synechocystis sp. 6803 (Table 1). From these genome-wide searches it is concluded that higher plants have a minimum consensus set of six Prx, (i) an embryogenesis-related 1-CysPrx, (ii) a cytosolic typeII Prx (Arabidopsis thaliana [At]PrxIIB-like), (iii) a mitochondrial typeII Prx (AtPrxIIF-like), (iv) a plastid typeII Prx (AtPrxIIE-like), (v) a chloroplast PrxQ, and (vi) a chloroplast 2-CysPrx (42). This basic pattern is subject to significant variation between plant species (Table 1); for example, $A$. thaliana expresses two highly similar 2-CysPrx, denominated At-2CysPrxA and At-2-CysPrxB, three cytosolic typeII Prx (PrxIIB, C, and D), and the peculiar PrxIIA whose functionality and expression are not entirely clear $(61,62)$. 
FIG. 1. Principle unified reaction mechanism of peroxide detoxification by Prxs. (A) The catalytic cycle consists of the three steps of peroxide reduction, thiol resolving, and dithiol regeneration. (B) Simplified schematic of the structure of the four Prx types in the oxidized state after step 2 of the reaction cycle. (a) According to the structure-based nomenclature 2-CysPrx belongs to the A-type Prx. The disulfide bridge is formed intermolecularly between the two subunits of each dimer. Each dimer has two catalytic centres. Both subunits dimerize by a Btype interface indicated by parallel orientation of the $\beta$-sheets. The rectangle with the $\beta$-sheets gives the orientation of the Prx subunit. (b) 1-CysPrx belongs to the B-type Prx with one catalytic Cys $\mathrm{P}_{\mathrm{P}}$ on both subunits that are linked by a B-type interface. (c) The C-type PrxQ acts as monomer with one $\mathrm{Cys}_{\mathrm{P}}$ and one $\mathrm{Cys}_{\mathrm{R}}$ that form an intramolecular disulfide bridge. (d) Type II Prx is assigned to the structural group of D-type Prxs and forms an intramolecular disulfide bridge during the catalytic cycle. The dimers assemble by a perpendicular A-type interface. The arrows in the squares indicate the orientation of the central $\beta$-sheets $(45,52)$.
A

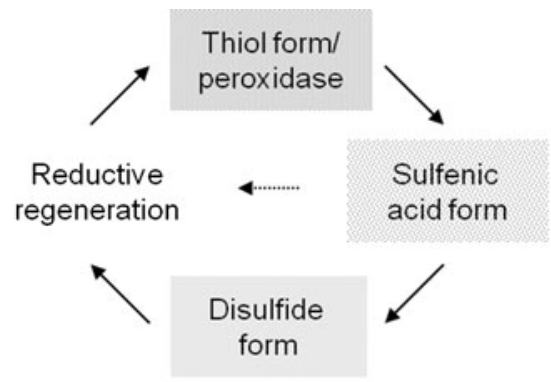

B

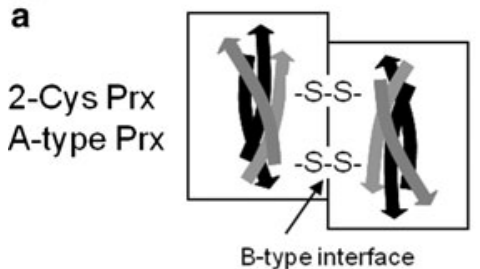

c
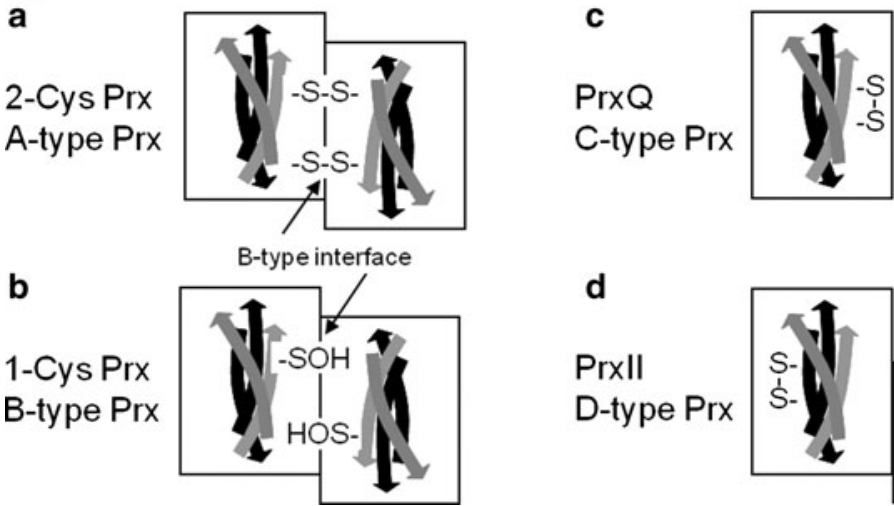

d

Prxll

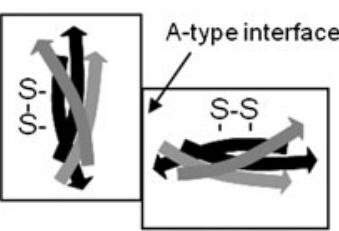

Figure 2 sorts the four types of eukaryotic Prx and in addition the group of glutathione peroxidases (Gpx) into a phylogenetic tree of sequence relatedness. The subfamilies are well distinguished and separated. The PrxII subfamily is diverse, due to early evolutionary separation into mitochondrial (PrxII F), plastidic (PrxII E), and cytosolic (PrxII B-D) forms. The genome survey of Bernroitner et al. (18) compares the antioxidant systems of simple and complex cyanobacteria: The small genomes of Prochlorococcus marinus genotypes only encode three Prx, that is, either one 2-CysPrx, one PrxQ, and one PrxII, or one 2-CysPrx and two PrxQ, whereas the genome of Nostoc punctiforme PCC73102 contains seven prx genes. $N$. punctiforme is a cyanobacterium with complex life cycle that includes differentiation of $\mathrm{N}_{2}$-fixing heterocysts, and hormogonia and akinetes for propagation (18). Including into our consideration the gene family sizes in yeast (five Prx) as well as human, mouse, and rat (six Prx) (57) the Prx family evolution shows tentative trends to increased member size in

Table 1. Prx Encoding Genes in Higher Plants, Cyanobacteria, and Brown Algae

\begin{tabular}{|c|c|c|c|c|c|c|}
\hline Prx gene & Localization & $\begin{array}{c}\text { Arabidopsis } \\
\text { thaliana } \\
\text { (MIPS) }\end{array}$ & Populus trichocarpa & $\begin{array}{l}\text { Oryza sativa } \\
\quad(T I G R)\end{array}$ & $\begin{array}{l}\text { Synechocystis } \\
\text { sp. PCC } 6803\end{array}$ & $\begin{array}{l}\text { Ectocarpus } \\
\text { siliculosus }\end{array}$ \\
\hline 1-CysPrx & N, Cyt & At1g48130 & POPTR_0008s09930 & $\begin{array}{l}\text { Os07g0638300 } \\
\text { Os07g0638400 }\end{array}$ & slr1198 & \\
\hline 1-CysPrx ${ }^{a}$ peptide & & & POPTR_0010s16260 & & & \\
\hline 2-CysPrxA & Chl & At3g11630 & POPTR_0016s07280 & Os02g0537700 & sll0755 & YP_003289214 \\
\hline 2-CysPrxB & Chl & At5g06290 & $\begin{array}{l}\text { POPTR_0006s22130 } \\
\text { (POPTR_1173s00210) }\end{array}$ & & & \\
\hline PrxQ1 & Chl & At3g26060 & POPTR_0006s13980 & Os06g0196300 & sll0221, slr0242 & \\
\hline PrxQ2 & Chl & & POPTR_0018s07400 & & & \\
\hline $\operatorname{PrxII} A^{a}$ & Cyt & At1g65990 & & & & \\
\hline PrxII B & Cyt & At1g65980 & POPTR_0001s44990 & $\begin{array}{l}\text { Os01g0675100 a } \\
\text { only one Cys }\end{array}$ & & \\
\hline PrxII C & Cyt & At1g65970 & POPTR_0018s09030 & & sll1621 & \\
\hline PrxII D & Cyt & At1g60740 & & & & \\
\hline PrxII E & Chl & At3g52960 & POPTR_0013s10250 & $\begin{array}{l}\text { Os06g0625500 } \\
\text { Os02g0192700 }\end{array}$ & & \\
\hline $\begin{array}{l}\text { PrxII F } \\
\text { PrxII }{ }^{\mathrm{a}} \text { peptide }\end{array}$ & Mit & At3g06050 & $\begin{array}{l}\text { POPTR_0019s04070 } \\
\text { POPTR_0012s07220 }\end{array}$ & Os01g0266600 & & \\
\hline
\end{tabular}

aputative Prx pseudo genes.

Chl, chloroplast/plastid; Cyt, cytosol; Mit, mitochondrion; N, nucleus. 


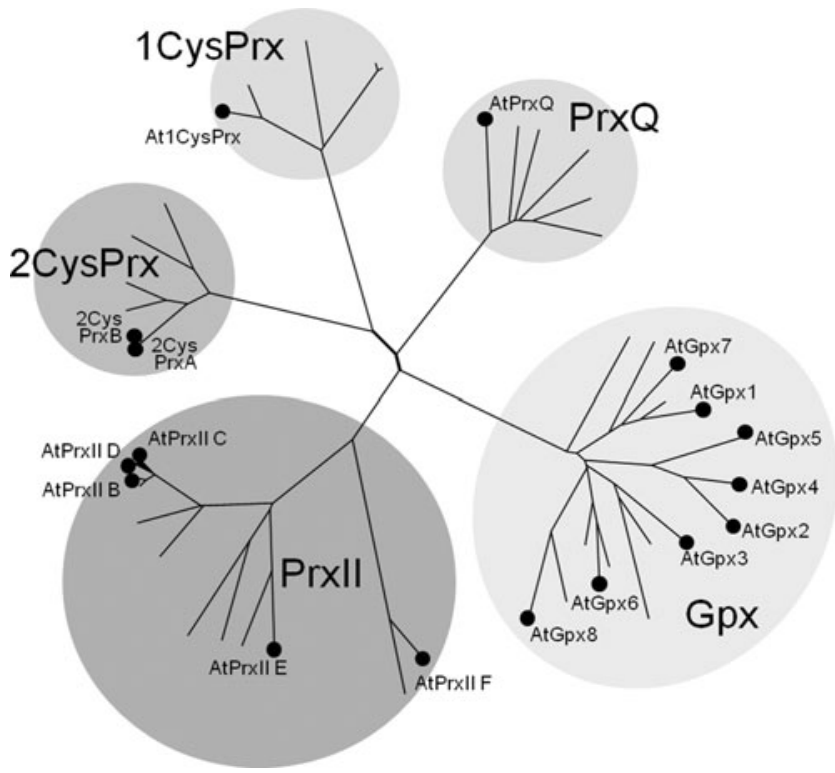

FIG. 2. Phylogenetic tree of Prx. Members of the four Prx groups, namely, 2-CysPrx, 1-CysPrx, PrxQ, and PrxII, and, in addition, the family of Gpx were clustered with Clustal W. The position of representative Prx members from Arabidopsis thaliana are indicated by ball-ended edges. Only protein sequences from higher plants were included in this analysis [cf. ref. (152)].

organisms with (i) larger genomes that coincides with (ii) more complex organization and (iii) oxygenic photosynthesis.

\section{Peroxidase Activity of Peroxiredoxins}

Prxs primarily function as thiol-dependent enzymes that decompose peroxides (57). Thus, they belong to the enzyme group of peroxidases (EC 1.11.1.) with the specific counting number 15 (EC 1.11.1.15). Their occurrence in essentially all organisms suggests that Prxs evolved as high-efficiency thiolperoxidases very early during life evolution. At that time Prxs most likely detoxified small amounts of ROS that were released by metabolism in the prevailing microaerobic atmosphere of the earth. The peroxidase activity was maintained during subsequent evolution, but in addition new Prx properties such as regulation of quaternary structure, chaperone activity, and hyperoxidation switch emerged later on particularly in eukaryotes as discussed below. The mature molecular mass of proper Prx ranges between 17 and $24 \mathrm{kDa}$ (150-220 amino acids). They catalyze the reduction of chemically diverse peroxides ranging from hydrogen peroxide, simple and complex alkyl hydroperoxides to peroxynitrite. In the literature Prxs have often been classified as low-efficiency peroxidases with low turnover number. Refined kinetic analyses changed this view since it was shown that the catalytic efficiency may be as high as for other enzymes like catalase, for example, in the case of bacterial alkylhydroperoxide reductase $\mathrm{C}(\mathrm{AhpC})$ time-resolved monitoring of the fluorescence decrease of the mutated electron donor $\mathrm{AhpF}$ in presence of hydroperoxides yielded a turnover rate of $53 \mathrm{~s}^{-1}$, a catalytic efficiency of ca. $4 \times 10^{7} 1 \mathrm{~mol}^{-1} \mathrm{~s}^{-1}$, and a 100 -fold preference for hydrogen peroxide over cumene hydroperoxide $(127,128)$. However, it should be noted that in the study in
2008 Parsonage et al. determined the redox midpoint potential of this bacterial Prx AhpC to be as high as $-178 \mathrm{mV}$. This characteristics might ease the catalytic turnover of this particular 2-CysPrx compared to other 2-CysPrx with much more negative midpoint redox potential (86). Two independent methods based on substrate competition of 2-CysPrx with horse radish peroxidase and monitoring peroxide-dependent disulfide bridge formation revealed a rate constant for hydroperoxide reduction of $1.3 \times 10^{7} 1 \mathrm{~mol}^{-1} \mathrm{~s}^{-1}$ for human peroxiredoxin 2 (Prdx2), a typical 2-CysPrx (135). Reported catalytic properties of 2-CysPrx vary considerably depending on experimental designs $(2,128,135,156)$. Detailed kinetic studies need also to be done for plant Prx. To complete the catalytic cycle after the peroxide reduction step (i) and the resolving step (ii), the sulfenic acid form of the catalytic Cys $\mathrm{P}$ (peroxidatic cysteine in the catalytic center) in case of 1CysPrx and the disulfide form of $\mathrm{Cys}_{\mathrm{P}}$ in case of 2-CysPrx need to be reduced (Fig. 3). This step (iii) is the regeneration step. Thioredoxins, glutaredoxins, glutathione, cyclophilins, and ascorbate function as electron donors to regenerate the active form of thiol peroxidase in a Prx-specific manner as described in more detail below [e.g., refs. $(86,89,92,151)]$.

Irrespective of whether the catalytic cycle proceeds in two or three consecutive reactions a specific cysteine residue in the active site is characteristic for all Prxs and essential for any peroxidase activity (peroxidatic cysteine, $\mathrm{Cys}_{\mathrm{P}}-\mathrm{SH}$ ). The

\section{(i) peroxide reduction step}

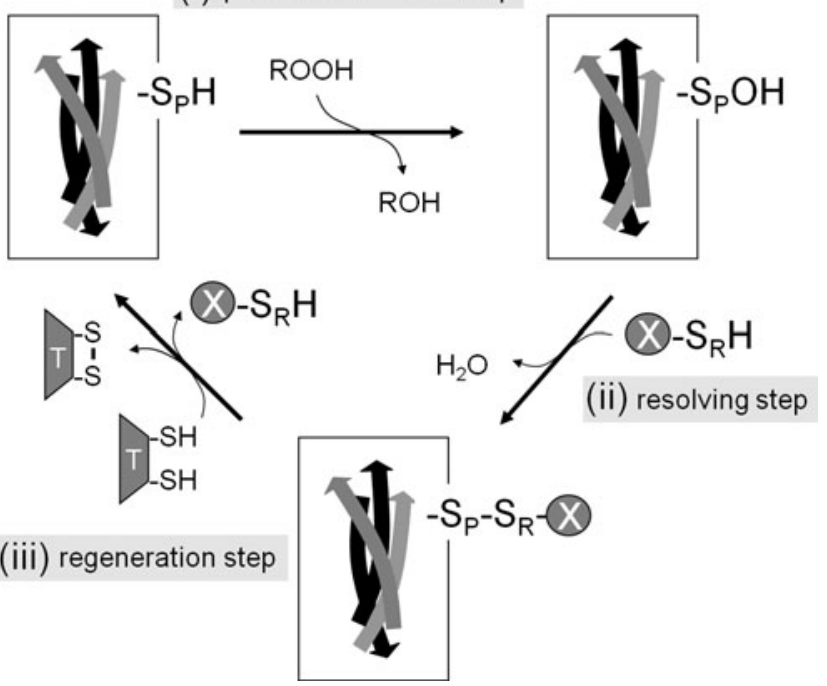

FIG. 3. Principle unified reaction mechanism of peroxide detoxification by Prxs. The generalized catalytic cycle of Prxs shown in Figure 1 consists of well-distinguishable chemical reactions: (i) the active thiol peroxidase reacts with the peroxide, (ii) the sulfenic acid derivative is formed, (iii) and further converted to the disulfide, and finally (iv) the active thiol is regenerated. In the first step the peroxide $\mathrm{ROOH}$ is reduced to the corresponding alcohol (or water in case of $\mathrm{H}_{2} \mathrm{O}_{2}$ or nitrite in case of $\mathrm{ONOO}^{-}$), which is released. The sulfenic acid derivative reacts with another resolving thiol $(X)$ to generate an inter- or intramolecular disulfide bridge. Water is released. The catalytically active form of the peroxide is restored after reaction with a thiol-oxidoreductase (T). The square with arrows represents the thioredoxin-like fold structure of Prx with its central $\beta$-sheets. $\mathrm{ONOO}^{-}$, peroxynitrite. 
thiolate of the $\mathrm{Cys}_{\mathrm{P}}-\mathrm{SH}$ attacks the peroxide substrate (Fig. 4). Mutagenesis of the cysteine results in loss of peroxidase activity of Prxs $(26,57,86,88,151)$. Conserved amino acids in the catalytic center, usually Pro-, Thr/Ser- (S/TXXC), and Arg/ Lys-residues, interfere with the $\mathrm{Cys}_{\mathrm{P}}-\mathrm{SH}$ and lower its $\mathrm{pKa}$ (124, 183). The Cysp-SH of typical 2-CysPrx such as from Salmonella typhimurium and E. coli AhpCs or yeast thiol-specific antioxidant range between 5.4 and 6.0, which is about 2 $\mathrm{pH}$ values lower than the unperturbed Cys-SH $(140,176)$. As a consequence, the thiol of $\mathrm{Cys}_{\mathrm{P}} \mathrm{S}^{-}$is highly active. The nucleophilic attack of the peroxide by $\mathrm{Cys}_{\mathrm{P}}-\mathrm{S}^{-}$results in the oxidation to $S$-hydroxycysteine (sulphenic acid, $\mathrm{Cys}_{\mathrm{p}}-\mathrm{SO}^{-}$) and the reduction of the hydrogenperoxide, alkyl hydroperoxide, and peroxynitrite to water, the corresponding alcohol and nitrite, respectively. A second free thiol acts as the resolving cysteine $\left(\mathrm{Cys}_{\mathrm{R}}-\mathrm{SH}\right)$ and reacts with $\mathrm{Cys}_{\mathrm{P}}-\mathrm{SO}^{-}$to form the disulfide $\mathrm{Cys}_{\mathrm{P}}-\mathrm{S}-\mathrm{S}-\mathrm{Cys}_{\mathrm{R}}$.

Cys $_{\mathrm{P}}$ is generally located in the $\mathrm{N}$-terminal protein part at about amino acid (aa) position 50, whereas the arrangement of the resolving cysteine $\left(\mathrm{Cys}_{\mathrm{R}}\right)$ varies between the Prx groups $(61,152)$ (Fig. 5). Cys ${ }_{R}$ of typical 2-CysPrx (group A Prx) locates in the C-terminal part at around aa 170 , for example, at aa 175 in the mature form of $A$. thaliana 2-CysPrxA. 2-CysPrx functions as obligate homodimer since the $\mathrm{Cys}_{\mathrm{P}}$ forms an intermolecular disulfide-bridge with the $\mathrm{Cys}_{\mathrm{R}}-\mathrm{SH}$ of the other subunit polypeptide chain. In the oxidized form the two 2CysPrx subunits are cross-linked by one or two disulfide bridges. The thiol-disulfide conversion of 2-CysPrx can be observed by band shifts in nonreducing SDS-polyacrylamide gels, and this allows for easy assessment of the redox state as long as other posttranslational modifications do not occur. There is no evidence that plant 2-CysPrx monomers exist in vivo or in vitro under native conditions. Only if reduced and denatured in the presence of urea or SDS, monomers are separated.

The recycling of $\mathrm{Cys}_{\mathrm{P}}-\mathrm{SOH}$ of 1-CysPrxs (Group B Prx) requires a resolving cysteine from another molecule or may proceed via ascorbate (see below). The other two Prx subgroups of plants form intramolecular disulfide bridges. PrxQ (Prx group C) and atypical 2-CysPrx (type II Prx; PrxII; Prx group D) share low amino acid sequence similarity and are also differentiated by the spacing of $\mathrm{Cys}_{\mathrm{P}}$ and $\mathrm{Cys}_{\mathrm{R}}$ on the same polypeptide by either 4 (PrxQ) or 24 amino acids (PrxII). More than 30 crystal structures from all four Prx types reveal that Prxs share the common and stable thioredoxin-like fold where seven $\beta$-sheets are surrounded by five $\alpha$-helices (123, 124). The conservation of the thioredoxin fold as central structural feature suggests that the Prx family evolved from a thioredoxin-like ancestral protein. Interestingly, the sequence similarity between the four Prx subgroups is low. PrxQ and atypical 2-CysPrx only share about 5\% of their amino acid sequence and the highest similarity is found between PrxQ and the typical 2-CysPrx subgroup with 34\% identity. On the other hand, similarity between vertebrate and plant 2-CysPrx is high with about $50 \%-60 \%$, and even slightly higher between plant and cyanobacterial and red algal 2-CysPrx $(7,82)$. This observation and in addition the fact that 2-CysPrx is still encoded by the plastome of Porphyra purpurea led to the evolutionary hypothesis that plant 2-CysPrx was introduced into plants via the endocytobiont that turned into the plastids (7).

Several amino acid residues of the active site are conserved among the different members of the Prx family (Fig. 5). X-ray crystallography and nuclear magnetic resonance analyses in combination with results from biochemical analyses of sitedirected mutagenized variants have identified the crucial amino acid residues required for catalytic mechanisms, structural flexibility, and oligomerization (52). The pKa of the active cysteine is lowered by the neighborhood of the catalytic triad consisting of Thr/Ser and Arg/Lys residues [for reviews see refs. $(140,176)]$. Further, a conserved Pro residue and hydrophilic residues shield $\mathrm{Cys}_{\mathrm{P}}$ from the bulk solvent. Trp and Phe residues are important for the conformational changes during the reaction cycle (87). The active site of the Gpx
FIG. 4. Schematic of the reaction mechanism with peroxide substrates of the different Prx types. (A) Typical 2CysPrx: The catalytic unit is a dimer. The peroxidatic thiol is deprotonated, acts as nucleophil, and attacks the peroxide, which is converted to the corresponding alcohol. The catalytic thiol is oxidized to sulfenic acid. The resolving thiol from the adjacent subunit forms a disulfide with $\mathrm{Cys}_{\mathrm{p}}$ and water is released. Thioredoxins and, in some special cases, Grx cyclophilins or GSH regenerate the catalytically active reduced form. (B) Atypical 2CysPrx: The reaction sequence is identical except of the position of the resolving Cys $_{R}$, which is located on the same subunit; thus, an intramolecular disulfide bridge is formed. (C) 1-CysPrx: Here the enzyme does not contain a $\mathrm{Cys}_{R}$. The sulfenic acid derivative directly reacts with a thiol or another reductant such as ascorbate.

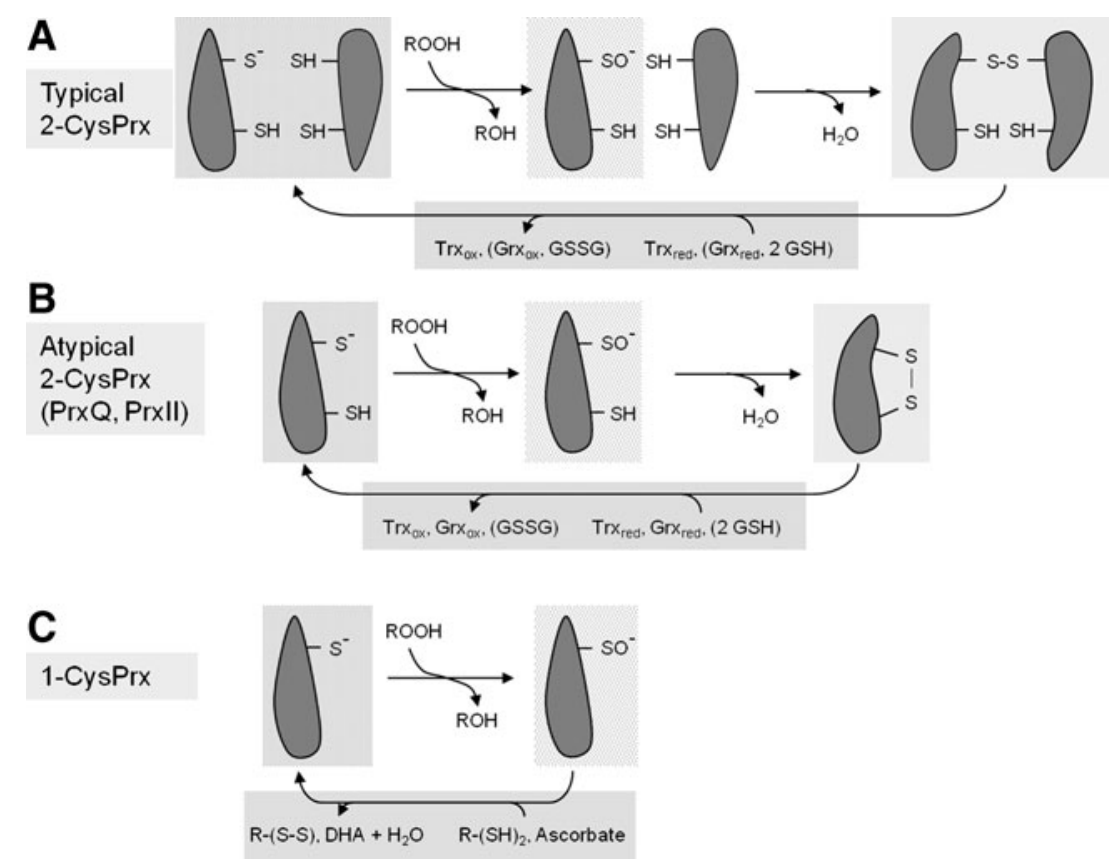




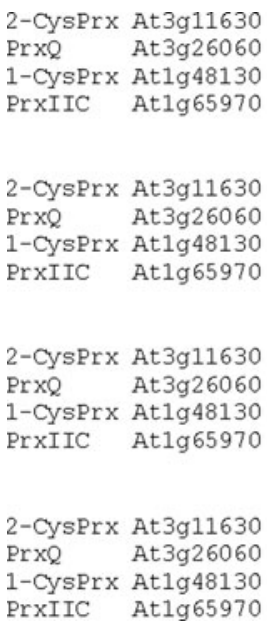

\section{----AQADDLPLVGNKAPDF EAEAV-FDQEF IKVKLSDY IGKKYVILFFYPLDFT FVCP 54 SSSLKGL IFAKVNKGQAAPDF TLK----DQNGKPVSLKKYKGKP-VVLYFYPADET PGCT 55 ------MPGIT LGDTVPNLEVET -----THDKEKLHDYFANSWTVLF SHPGDFT PVCT 47 ------MAPITVGDVVP DGT ISF FDENDQLQTVSVHS IAAGKKVILFGVPGAFT PTCS 52

$$
{ }^{\star}: .{ }^{\star}: \quad . .: \text {. . . . }{ }^{\star}:{ }^{\star}{ }^{\star} \text {. }
$$

T-EITAF SDRHSEFEKLNT-EVLGV SVDSVF SHLAWVOTDRKSGGLGDLNYPLISDVTKS 112 K-QACAF RDSYEKF KKAGA-EVIGI SGDDSASHKAFASKYK-------LPYTLLSDEGNK 106 T-ELGAMAKYAHEF DKRGV-KLLGL SCDDVQSHKDWIKDIEAFNHGSKVNYPIIAD PNKE 105 MSHVPGF IGKAEE LKSKGIDE I ICF SVND PFVMKAWGKTY PE-----NKHVKFVADGSGE 107

$$
\text { . . : }:: . . \quad::: \text {. }^{*}: . \quad: \text {. } \quad:::^{*} \text {. }
$$

ISKSFGV LIHDQG-IALRGLF I IDKEGVIQHSTINNLGIGRSVDETMRT LQALQYIQENP 171 VRKDWGV PGDLFGALPGRQTYVLDKNGVVQLIYNNQFQPEKHI DET LKF LKAA------- 159 IIPQLNMIDPIEN-GPSRALHIVGPDSKIKLSFLYPSTTGRNMDEVLRALDSLLMASKHN 164 YTHLLGLELDLKD----KGLGIRSRRFALLLDNLKVTVANVESGGEF TVSSAEDILKAL- 162

DEVC-PAGWKPGEKSMKPDPKLSKE YFSAI-------------- 200

NKIATPVNWKPDQPVVISPAVSDEEAKKMF PQGF KTADLPSKKGYLRHTEVS 216
FIG. 5. Alignment of the mature forms of the four Prx types of A. thaliana. PrxII C was selected to represent the type II Prx. "**" means that the residues in that column are identical in all sequences in the alignment, ":" means that conserved substitutions have been observed, and "." means that semiconserved substitutions are observed. The pairwise alignment was done with ClustalW at www.ebi .ac.uk with the default settings in the slow mode (29). family differs from the one of the Prx family, suggesting different ancestors. The similar reaction mechanism may be considered as an example of convergent evolution. For this reason, the group of glutathione-peroxidase-like plant proteins will not be deeply discussed in this review despite the suggestion of categorizing them as fifth subtype of Prx (120).

\section{Conformational Dynamics and Interacting Partners of Prx In Vitro and In Vivo}

The tertiary and quaternary structures of Prx proteins depend on the redox state of the catalytic peroxidative cysteine $\mathrm{Cys}_{\mathrm{P}}$. In terms of functional unit during catalysis and as dicussed above (Fig. 4) 1-CysPrx, PrxQ, and PrxII act as monomers, whereas 2-CysPrxs function as obligate dimers. However, the regular quaternary assembly appears to be the dimer for both 2-CysPrx and 1-CysPrx, whereas based on results from size exclusion chromatography PrxQ and PrxII are reported either as monomers or dimers, or a mixture of both $(14,86,133,169)$. Some Prx dimers assemble to higher molecular mass complexes such as hexamers, decamers, dodecamers, dodecahedrons, and tube-like structures (14) (Fig. 6). In addition, many Prx interact with protein partners. Experimental and in silico approaches to elucidate and func- tionally understand these interactions in vitro and in vivo presently emerge as an important field in redox network understanding. In addition to pull down assays and yeast-2hybrid screenings, methodological advances over the last years enable to address the composition and function of the cellular thiol-disulfide redox regulatory network, and the qualitative and quantitative interactions between its components. Prxs are part of the redox regulatory network of the cells and have been suggested to function as ROS traps and redox sensors (39) (Fig. 7). Essentially Prxs react with peroxides, turn oxidized and during reductive regeneration drain electrons from their respective electron donor (electron transmitter in Fig. 7). At this point the pioneering work of Arne Holmgren should be mentioned, which initially developed biochemical approaches to identify thioredoxin (Trx) target proteins $(58,59)$. It is suggested that the competition for electrons causes oxidation of other target proteins and regulates activities of components of the dithiol-disulfide redox network (39). The nature of protein targets within the thioldisulfide network was addressed at the proteomics scale. Both, thioredoxins and glutaredoxins were modified by Cys $\rightarrow$ Ser mutations to abolish the activity of the resolving cysteine $\mathrm{Cys}_{\mathrm{R}}(114,178)$. After covalent bonding of these Trx and glutaredoxin (Grx) variants to chromatographic material,
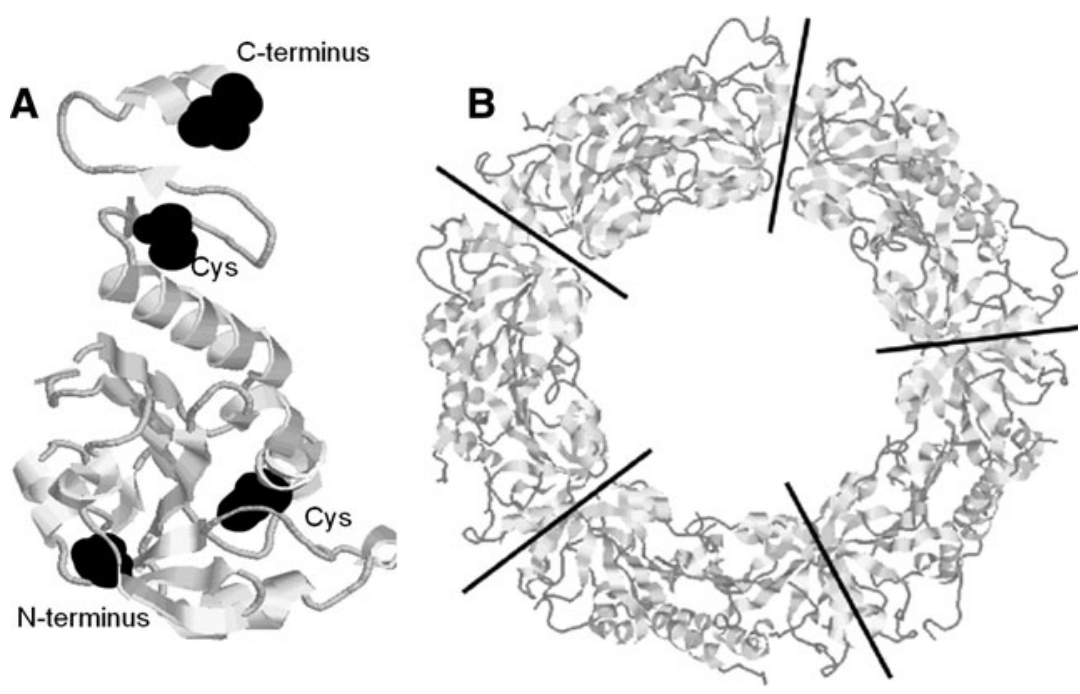

FIG. 6. Structure of 2-CysPrxA. (A) Monomer: The conserved cysteine residues are depicted as are the $\mathrm{N}$ - and $\mathrm{C}$-terminal amino acids as indicated. (B) Arrangement of the five dimers in the decamer of 2CysPrxA (At3g11630). The model was generated with RasMol software based on the structure of 1qmvG (identity $63.5 \%$ ) and processed using the Deep View/ Swiss-Pdb Viewer 3.7. 
FIG. 7. Function of Prx within the dithiol-disulfide redox network of the chloroplast. Photosynthesis via ferredoxin and nonphotosynthetic metabolism via NADPH provide electrons to the network. A third branch is linked to glutathione as redox input elements. Thioredoxin, NTRC, and Grx act as redox transmitters and donate electrons to redox target proteins whose activity is controlled and which themselves regulate diverse processes. In addition the redox transmitters donate electrons to specific Prx. ROS produced in metabolism accepts electrons from target proteins and oxidizes them. To avoid this uncontrolled reaction, Prx act as efficient ROS traps. They may function as redox sensors transferring redox information to the redox transmitters in a controlled manner [cf. ref. (42)]. Information on specific electron donors to PrxQ and 2-CysPrx is found in the text. 1-CysPrx was omitted from this figure because of its particular location in the nucleo-cytoplasm. Fd, ferredoxin; GSH, glutathione; NTRC, NADPH-dependent thioredoxin reductase C; ROS, reactive oxygen species.

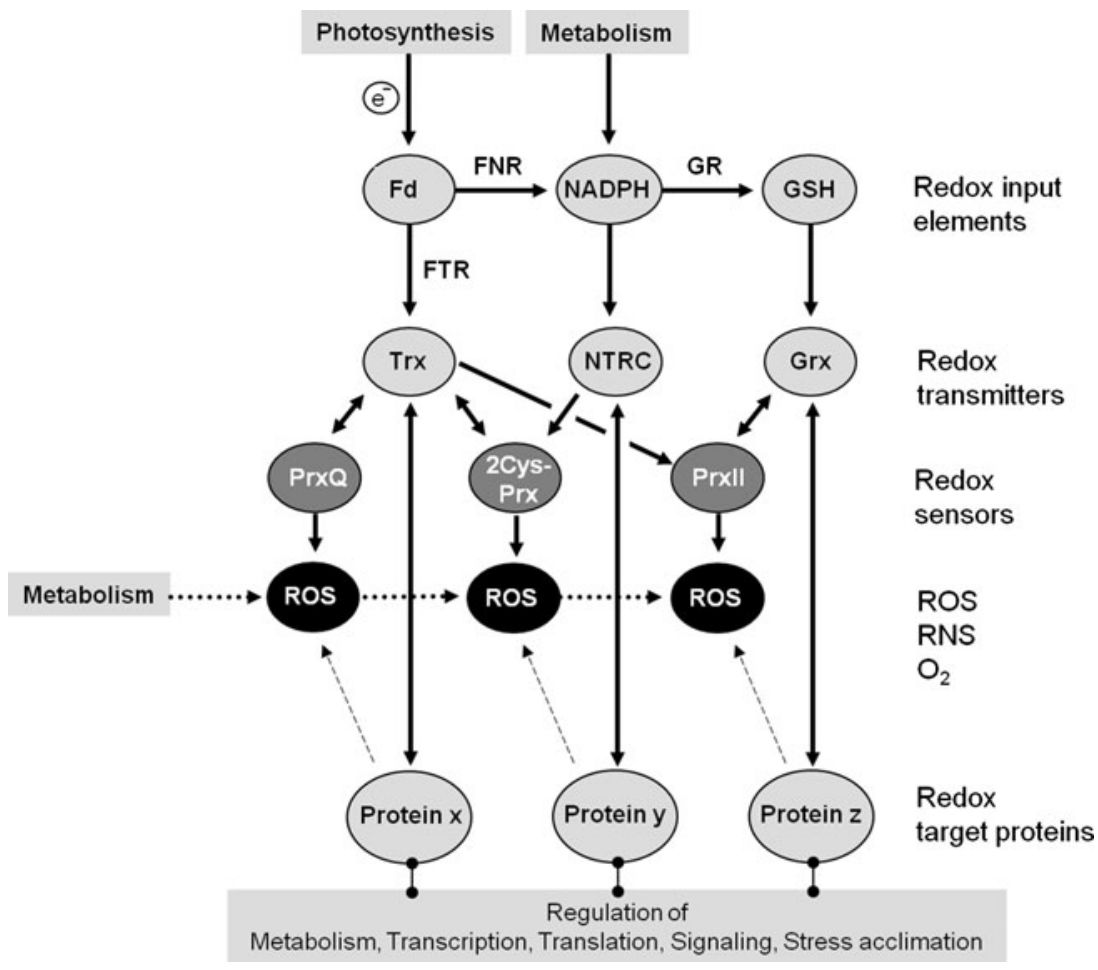

CysPrx-YFP and 2-CysPrx-CFP when simultaneously introduced in the cells. The FRET efficiency distribution in this experiment changes in the presence of dithiothreitol or $\mathrm{H}_{2} \mathrm{O}_{2}$, indicating redox-dependent dynamics of 2-CysPrx conformation in vivo. Two-step FRET enables analysis of interactions of three and more interactors. Simultaneous cotransfection of protoplasts with 2-CysPrx fused to CFP, YFP, and mCherry, respectively, enables radiationless energy transfer from CFP via YFP to mCherry (two-step FRET). Occurrence of two-step FRET depends on close proximity of three flagged subunits and in the case of 2-CysPrx indicates existence of oligomeric 2CysPrx in the chloroplast (159). These data sets prove that Prxs are parts of functional modules that may form stable but also dynamic complexes in dependence on redox environment and interacting partners.

\section{Typical 2-Cys Peroxiredoxins}

\section{A. Characteristics of 2-CysPrxs}

The group of typical 2-CysPrxs is characterized by the highest degree of sequence conservation among the four Prx groups present in eukaryotes. Four of the six Prxs in mammalian systems are 2-CysPrx (PrxI-IV), whereas mammalian genomes only encode one PrxII (Prx V) and one 1-CysPrx (Prx VI). Interestingly, the recently sequenced genome of the brown alga Ectocarpus siliculosus appears to code for only one Prx gene representing a typical 2-CysPrx and thus lacks other Prx types (34). Alternatively, additional Prx may be encoded by organellar subgenomes. In plants, the 2-CysPrx is targeted to the chloroplast (7) where a significant fraction associates with the thylakoid membrane in a redox state-dependent manner (86). The N-terminal domain carries the typical features of transit peptides. 2-CysPrxs exhibit conserved motifs

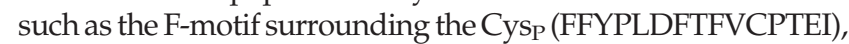

action with Trx-x is either too short-lived or absent to detect FRET (118). In addition, one-step FRET occurs between 2- of YFP fluorescence only occurs if both partners are in close proximity in vivo. Measured FRET efficiencies of 18\%-20\% indicate interaction of 2-CysPrx with Cyp20-3 and NADPHdependent thioredoxin reductase C (NTRC), whereas inter- 
the motif in the vicinity of the $\mathrm{Cys}_{\mathrm{R}}$ (EVCP) and the GGLG and YF motifs found in eukaryotic Prxs, which are involved in sensitivity toward hyperoxidation (182). After reacting with the first peroxide substrate to the intermediary sulfenic acid, hyperoxidation of $\mathrm{Cys}_{\mathrm{P}}$ to sulfinic or sulfonic acid derivatives occurs when the resolving reaction is too slow to form the disulfide before a second peroxide attacks. Reported catalytic efficiencies $\left.\mathrm{k}_{\mathrm{cat}} / \mathrm{K}_{\mathrm{M}}\left[1 \mathrm{~mol}^{-1} \mathrm{~s}^{-1}\right)\right]$ of plant 2-CysPrx with peroxide was $2.5 \times 10^{4}$ with the artificial regenerator dithiothreitol in pea [ref. (17); $\mathrm{K}_{\mathrm{M}} 27.6 \mu \mathrm{mol} \mathrm{H}_{2} \mathrm{O}_{2} \mathrm{l}^{-1}$; $\mathrm{k}_{\text {cat }} 0.69 \mathrm{~s}^{-1}$, with $4 \mathrm{mM}$ DTT], $1.8 \times 10^{3}$ with Trx-x in Oryza sativa [ref. (133); $77.4 \mu \mathrm{mol}$ tertiary butylhydroperoxide (tBOOH) $1^{-1} ; \mathrm{k}_{\mathrm{cat}}$ $0.14 \mathrm{~s}^{-1} ; 7.5 \mu M$ Trx-x] and $2.1 \times 10^{5}$ with NTRC [ref. (133); $21.8 \mu \mathrm{mol} \mathrm{tBOOH} 1^{-1} ; \mathrm{k}_{\text {cat }} 4.62 \mathrm{~s}^{-1}, 1 \mu \mathrm{M}$ NTRC]. Thus, NTRC seems to most efficiently reduce plant 2-CysPrx, but as mentioned above, maximal catalytic efficiencies of plant 2CysPrx still need to be determined, for example, by stop flow or other time-resolved methods.

2-CysPrx functions as homodimer $\left\{\alpha_{2}\right\}$ as elementary structural and catalytic unit. The core structure of each Prx subunit is a sevenstranded $\beta$-sheet typical for Trx fold proteins. The plane of the $\beta$-sheets orientates parallel to the interface of both subunits and is categorized as B-type interface (see above: Fig. 1B). Almost all 2-CysPrx homodimers have a high propensity to assemble to higher molecular mass structures, most commonly to decamers $\left\{\left(\alpha_{2}\right)_{5}\right\}$. Dimers interact with interfaces roughly perpendicular to the central $\beta$-sheet planes. These interfaces are considered as A-type interfaces (52). The dimer-dimer interaction involves hydrophobic contact sites. Plant 2-CysPrxs exhibit redox-dependent dynamics in tertiary and quaternary structure. As a consequence 2-CysPrxs adopts at least five distinct conformations under control of the redox state and other posttranslational modifi- cations (Fig. 8), namely, the conformations of (i) the oxidized dimer, (ii) the reduced dimer, (iii) the reduced decamer, (iv) the hyperoxidized decamer, and (v) higher mass aggregates of hyperoxidized decamers (118). The structural basis for the high propensity to hyperoxidation was approached by amino acid sequence comparisons between eukaryotic 2-CysPrx prone to hyperoxidation and prokaryotic 2-CysPrxs, which are highly resistant to hyperoxidation. A GGLG motif and the C-terminal helix with its buried YF motif are involved in a tighter structure of the fully folded form, slowing down the disulfide bridge formation during the catalytic cycle and increasing the propensity for hyperoxidation (182). There is broad acceptance of the hypothesis that the hyperoxidation evolved to enable redox-dependent ROS signaling, for example, to the nucleus under conditions of excessive peroxide formation. Recently, Pascual et al. (129) showed that 2CysPrxs from some prokaryotes, including cyanobacteria, contain the GG(L/V/I)G and YF motifs characteristic of sensitive enzymes. 2-CysPrxs from the cyanobacterium Anabaena PCC7120 and Synechocystis sp. PCC6803 convert to the hyperoxidized form when reductant and high levels of peroxides are present simultaneously. The authors described a high similarity between the chloroplast and Anabaena antioxidant systems, which employ a hyperoxidation-sensitive 2CysPrx and lack catalase. This mechanism may allow for the transient increase of $\mathrm{H}_{2} \mathrm{O}_{2}$ to act as messenger in retrograde signaling (129).

\section{B. Reduction of oxidized 2-CysPrx}

Plastids contain a thiol-disulfide redox network composed of input elements, redox transmitters, and target proteins (Fig. 7). The state of the network is defined by the balance between

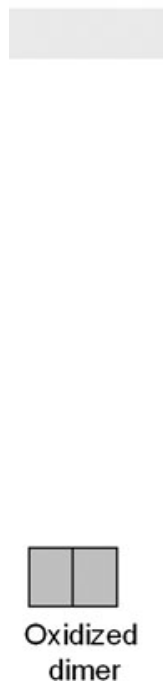

\section{Peroxidase}
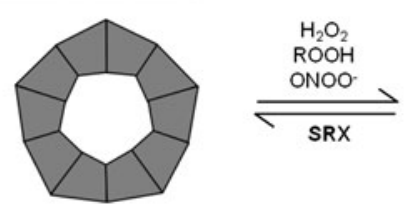

Reduced

decamer
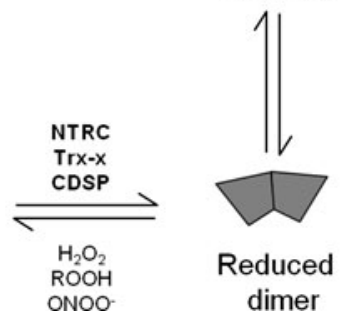

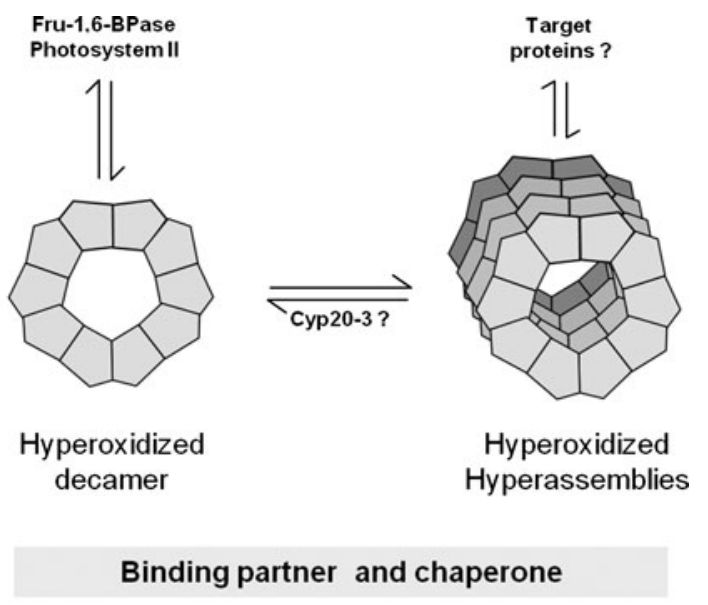

FIG. 8. Conformational states of 2-CysPrx and concomitant switching between peroxidase, binding partner, and chaperone activity. The reduced dimer and decamer function as peroxidase. The oxidized dimer is regenerated by several electron donors, including NTRC, Trx-x, and CDSP32. After hyperoxidation a stable decamer is formed that structurally differs from reduced decamer. Hyperoxidized decamers assemble to high-molecular-mass aggregates that function as chaperones. In addition 2-CysPrx acts as binding partner [cf. ref. (118)]. 
electron feeding into the network and drainage of electrons by $\mathrm{O}_{2}$, ROS, RNS, and metabolism. The plastids contain a large set of at least 11 thioredoxins in the stroma, namely, Trx-f1, f2, $\mathrm{m} 1-\mathrm{m} 4, \mathrm{x}, \mathrm{y} 1, \mathrm{y} 2, \mathrm{z}$, chloroplast drought-induced stress protein (CDSP), and, in addition, the NTRC protein, which is a dual-domain protein of NADPH-dependent thioredoxin reductase and a thioredoxin domain (105). NTRC was also identified as interactor of 2-CysPrx in a yeast-2-hybrid screen using 2-CysPrx as bait. Subsequent biochemical work revealed that NTRC efficiently donates electrons to 2-CysPrx (110). Collin et al. (35) compared Trx-m1, -m2, -f1, -f2, and -x for their efficiencies to regenerate 2-CysPrx. $\mathrm{K}_{\mathrm{M}}$-values for Trx-interaction ranged between 25 and $279 \mu \mathrm{M}$, and thus their affinities appear to be quite low and limiting. The catalytic efficiency with $\mathrm{tBOOH}$ as substrate and Trx as regenerant reaches values between $0.33 \times 10^{3}$ and $7.3 \times 10^{3} 1 \mathrm{~mol}^{-1} \mathrm{~s}^{-1}$ (35), but was $2.1 \times 10^{5} 1 \mathrm{~mol}^{-1} \mathrm{~s}^{-1}$ for NTRC and 2-CysPrx in rice (133). Figure 9 summarizes present-day knowledge on the position of chloroplast 2-CysPrx within the dithiol-/disulfide redox regulatory network and the proposed electron flow into and electron drainage from the 2-CysPrx system. Various studies indicate that Trx- $x$ and NTRC function as important electron donors, whereas additional donors such as Trx-f1, Trx-m1, CDSP32, and cyclophilin Cyp20-3 may serve as ancillary low-efficiency electron donors. Stimulated ROS production in stressed potato induces accumulation of the two-Trx modules carrying atypical thioredoxin termed CDSP32 (22). CDSP32-deficient potato transformants are more sensitive than wild type to oxidative stress treatments induced by application of methyl viologen or butylhydroperoxide $(21,23)$. The turnover achieved in the tests was close to $3 \mathrm{~mol} \mathrm{H}_{2} \mathrm{O}_{2}$ (mol CDSP32 $\left.\mathrm{min}\right)^{-1}$ and thus lower than the values reported for 2-CysPrx regeneration with $\operatorname{Tr} x-x$ or NTRC $(35,133)$. It should be noted that reduction of CDSP

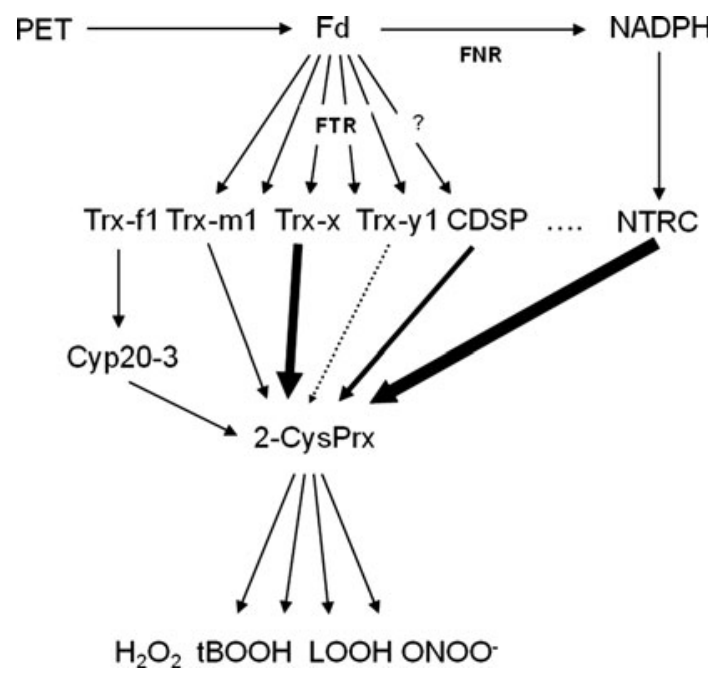

FIG. 9. 2-CysPrx as hub in the redox regulatory network of the chloroplast. 2-CysPrx accepts electrons from several redox transmitters and detoxifies different peroxides. The thickness of the edges tentatively denotes the efficiency of electron transfer. FNR, Fd-dependent NADP reductase; FTR, Fd-dependent thioredoxin reductase; PET, photosynthetic electron transport. and Trx- $x$ by ferredoxin-dependent thioredoxin reductase has not experimentally been demonstrated.

To assess the relative contribution of Trx- $x$ and NTRC to 2CysPrx reduction in vivo, Pulido et al. (142) compared the effect of deleting either one of both oxidoreductase systems in A. thaliana. While tr $x-x$ knock out plants are similar to wild type and maintain a fraction of 2-CysPrx in the reduced state, $n t r c$ knock out plants accumulate only oxidized 2-CysPrx. In line with this result, extracts from wild-type and tr $x-x$ plants contain similar $\mathrm{H}_{2} \mathrm{O}_{2}$ amounts $(0.77 \mu \mathrm{mol} / \mathrm{g}$ fresh weight), whereas $\mathrm{H}_{2} \mathrm{O}_{2}$ levels increase to 0.99 in $n$ trc plants (142). This assigns the predominating function in reductive regeneration of chloroplast 2-CysPrx to the NTRC system as suggested before by Kirchsteiger et al. (80).

\section{Consequences of 2-CysPrx deficiency}

Artificially induced 2-CysPrx deficiency in plants and cyanobacteria has been established by an antisense approach (8), by crossing two transfer DNA (T-DNA) lines carrying insertions in each of the two 2-CysPrx A and B genes of $A$. thaliana (142), and by insertional inactivation in Synechocystis PCC6803 $(82,185)$. It should be noted that a full knock out line lacking both 2-CysPrxA and 2-CysPrxB has not yet been established. The $A$. thaliana antisense lines showed severe 2-CysPrx deficiency during early plant development from germination to an age of about 4 weeks. Later on 2-CysPrx accumulated to wild-type levels (8). Accordingly, altered phenotypes of inhibited growth and chlorosis were only seen in young plants and seedlings, particularly when grown on agar plates. Pulido et al. (142) crossed two T-DNA insertion mutants of 2-CysPrxA and B to obtain $42 \mathrm{cp}$. The mutant contains no detectable amounts of 2-CysPrx B transcripts and a very low 2-CysPrx $A$ transcript level. Total 2-CysPrx protein accumulates to levels of about or below $5 \%$ of wild type. Biomass accumulation of the $\Delta 2 c p$ is inhibited by about $25 \%$ compared to wild type. The $\Delta 2 c p$ plants have lower photosynthetic pigment contents, such as total chlorophyll and carotenoids (142). The most severe effect of 2-CysPrx deficiency as measured by the dinitrophenyl hydrazine method is seen on protein carbonylation, which is increased 4.7 -fold in $\Delta 2 c p$ $(321.8 \pm 62.4)$ compared to wild type $(68.8 \pm 13.1 \mathrm{nmol}$ dinitrophenyl hydrazine $\mathrm{mg}^{-1}$ protein). This indicates severe oxidative stress despite the compensatory activation of antioxidant defense enzymes (9). This compensation is reflected by increased transcript accumulation and activities of stromal and thylakoid ascorbate peroxidase as well as monodehydroascorbate reductase (9). Attention in context of 2CysPrx function was also given to cyanobacteria, which are considered as model systems to investigate mechanisms in and regulation of oxygenic photosynthesis. Synechocystis PCC6803 carrying an insertion in the 2-CysPrx gene (sll0755) grows slower than wild type in full strength medium. The inhibition is overcome in media with lowered Fe and micronutrient contents (82). 2-CysPrx lacking Synechocystis PCC6803 cannot support $\mathrm{H}_{2} \mathrm{O}_{2}$ - and $\mathrm{tBOOH}$-dependent oxygen evolution in the light (185). Tichy and Vermaas (173) analyzed the $\mathrm{H}_{2} \mathrm{O}_{2}$ detoxification capacity of Synechocystis PCC6803 and detected only two peroxidative activities, namely, that of the catalase peroxidase KatG and the thioldependent peroxidase Prx. The Prx-dependent activity is light dependent and sufficient to support wild type like growth of 
Synechocystis PCC6803 even in the absence of KatG. Table 2 compiles results from diverse genetic approaches undertaken to analyze the function of specific Prxs in higher plants or cyanobacteria. In each case modification of 2-CysPrx amounts affected traits related to photosynthesis. The other experiments dealing with PrxQ, 1-CysPrx, and PrxII will be discussed below.

\section{Peroxiredoxin Q, an Atypical 2-CysPrx of Chloroplasts and Cyanobacteria}

PrxQ homologs (bacterioferritin comigratory protein, bacterioferritin comigratory protein; atypical 2-Cys Prx) are found in bacteria (Bcp protein) and some lower eukaryotes but are absent from mammals. PrxQ was the last member of the Prx family identified in plants (85). PrxQ is characterized by the positioning of its catalytic and resolving Cys-residues both in a single polypeptide spaced by four amino acids in SlPrxQ or At-PrxQ (Fig. 5). Thus, in Sedum lineare PrxQ Cys44 and Cys49 are essential for the thioredoxin-dependent peroxidase activity. Heterologous S1-PrxQ expression suppresses the peroxide hypersensitivity of an E. coli bcp mutant, tentatively confirming its antioxidant activity in vivo. PrxQ functions as monomeric peroxidase with high reactivity toward $\mathrm{H}_{2} \mathrm{O}_{2}$ and butylhydroperoxide, and still significant activity toward lipid peroxides. Members of PrxQ type often constitute small gene families in cyanobacteria with significant variation in arrangement of Cys residues $(169,170)$. Two out of the five Prx encoded in the Synechocystis PCC6803 genome are PrxQ-like gene products, whereas the genome of $S y$ nechococcus elongatus PCC7942 contains four prxQ-like genes, namely, prx $Q-A 1,-A 2,-A 3$, and $B$ (170). Viability of $E$. coli BL21 cells expressing the cyanobacterial PrxQ-A1, A2, or A3 improves in the presence of toxic cumenehydroperoxide concentrations, whereas expression of the other PrxQ-B has no effect (169). This suggests a significant detoxification function of cyanobacterial Prx-Q. Interestingly, while the Cys $P$ is conserved among the various members of the cyanobacterial PrxQ families, the $\mathrm{Cys}_{\mathrm{R}}$ usually located at position 5 downstream of $\mathrm{Cys}_{\mathrm{P}}$ is lacking from, for example, PrxQ-A of Anabaena PCC7120, PrxQ-B1, and -B2 of Synechocystis PPC 6803 and PrxQ-B of Synechococcus elongatus PCC7942. The

Table 2. Compilation of Transgenic Approaches with Modified Prx Amounts

\begin{tabular}{|c|c|c|c|c|}
\hline Prx type & Species & Approach & Phenotype & Reference \\
\hline 2-CysPrx & A. thaliana & Antisense suppression & $\begin{array}{l}\text { Phenotype of young plants: slow } \\
\text { growth; inhibited photosynthesis; } \\
\text { chlorosis, compensatory } \\
\text { regulation of antioxidant defense }\end{array}$ & 8 \\
\hline 2-CysPrx A/B & A. thaliana & Insertion line & $\begin{array}{l}\text { Lower biomass, lower } \\
\text { photosynthesis; increased protein } \\
\text { carbonylation }\end{array}$ & 142 \\
\hline 2-CysPrx & Festuca arundinaceae & Overexpression & $\begin{array}{l}\text { Tolerance to heat stress and methyl } \\
\text { viologen }\end{array}$ & 46 \\
\hline 2-CysPrx (sll0755) & Synechocystis PCC6803 & Insertional inactivation & $\begin{array}{l}\text { Slow growth in full strength } \\
\text { medium }\end{array}$ & 82 \\
\hline 2-CysPrx (sll0755) & Synechocystis PCC6803 & Insertional inactivation & $\begin{array}{l}\text { Deficient in peroxide dependent } \mathrm{O}_{2} \\
\text { evolution }\end{array}$ & 183 \\
\hline $\operatorname{PrxQ}$ & A. thaliana & Insertion line & $\begin{array}{l}\text { Decreased oxidative stress } \\
\text { sensitivity; changed stoichiometry } \\
\text { of photosynthetic components }\end{array}$ & 88 \\
\hline PrxQ & A. thaliana & Insertion line & $\begin{array}{l}\text { Slightly increased photosynthesis } \\
\text { and chlorophyll a/b-ratio }\end{array}$ & 137 \\
\hline PrxQ & Nicotiana tabacum & Overexpression of GtPrxQ & $\begin{array}{l}\text { Resistance to methylviologen and } \\
\text { Botrytis infection }\end{array}$ & 77 \\
\hline PrxQ & A. thaliana & $\begin{array}{l}\text { Overexpression of Suaeda } \\
\text { salsa-PrxQ }\end{array}$ & $\begin{array}{l}\text { Increased tolerance to salt and cold } \\
\text { stress }\end{array}$ & 72 \\
\hline 1-CysPrx & A. thaliana & RNAi & Early germination under stress & 55 \\
\hline 1-CysPrx & A. thatiana & overexpressor & $\begin{array}{l}\text { Delayed germination under abiotic } \\
\text { stress }\end{array}$ & 55 \\
\hline PrxII (sll1621) & Synechocystis PCC6803 & Insertional inactivation & Slow growth & 64 \\
\hline PrxII (sll1621) & Synechocystis PCC6803 & Insertional inactivation & High light sensitivity & 83 \\
\hline PrxIIF & A. thaliana & Insertion line & $\begin{array}{l}\text { Root growth inhibition in presence } \\
\text { of Cd and salicylhydroxamic acid; } \\
\text { compensatory upregulation of } \\
\text { APX and GPx }\end{array}$ & 46 \\
\hline PrxIIE & A. thaliana & Insertion line & $\begin{array}{l}\text { Increased protein nitration; } \\
\text { suggested role in pathogen } \\
\text { defense }\end{array}$ & 148 \\
\hline PrxIIE & A. thaliana & Overexpressing line & $\begin{array}{l}\text { Decreased protein nitration after } \\
\text { ONOO }^{-} \text {-infiltration }\end{array}$ & 148 \\
\hline
\end{tabular}

The table summarizes transgenic experiments where Prx amounts have specifically been manipulated either in higher plants or cyanobacteria. Given are Prx type, species, introduced genetic modification, phenotype and reference. 
latter PrxQ-B lacks any other Cys-residue, whereas the other three sequences contain Cys residues at nonconserved positions $(169,170)$. The mechanism of the catalytic cycles of these atypical PrxQ is unknown. The variability of PrxQ primary structure and Cys arrangements suggests that in addition to typical and atypical representatives of 2-CysPrx, one has to distinguish between typical and atypical 1-CysPrx as well. PrxQ isoforms with single Cys residues should be assigned to the new group of atypical 1-CysPrx. Amino acids involved in the catalytic activity were addressed by site-directed mutagenesis of At-PrxQ. Arg181 appears to participate in the primary catalytic mechanism, Arg118 in conformational changes needed for accessing the catalytic site, and Thr107 and Thr111 in Trx-mediated regeneration (88). The results indicate mechanistic similarities between PrxQ and the related 2CysPrx (87).

Plant PrxQ interacts with thioredoxins as first suggested by a proteomics approach where site-directed mutagenized spinach Trx-m lacking its resolving Cys was immobilized on cyanogen bromide-activated sepharose $(114,115)$. This analysis as that of Rey et al. (146) used stromal fractions of spinach and potato, respectively, for their proteomics screening. Target proteins are trapped on the column under oxidative conditions since the intermolecular disulfide bridges formed between Trx-m and the targets are not resolved due to the mutation of the second $\mathrm{Cys}_{\mathrm{R}}$. Among the mass-spectrometrically identified proteins were well-known targets of chloroplast redox regulation such as glyceraldehyde-3-phosphate dehydrogenase and sedoheptulose-1,7-bisphosphatase, and also At-2CysPrx, PrxQ and Cyp20-3 (114). Chloroplast location of Prx-Q was first shown by Rouhier et al. (149) in poplar. While thioredoxins are able to reduce Populus tremula PrxQ (Pt-PrxQ), various glutaredoxins and cyclophilin fail to support reductive regeneration of oxidized PrxQ $(89,149)$. The redox midpoint potential of the PrxQ catalytic dithiol/ disulfide transition is $-325 \mathrm{mV}(\mathrm{pH} 7.0)$. In a more detailed study, Trx-y2, which is expressed in photosynthetic tissues, efficiently reduced $\operatorname{Pr} x Q$, whereas it failed to effectively activate malate dehydrogenase and fructose-1,6-bisphosphatase (36). The newly identified At-Trx-z, which is associated with the transcriptional machinery of the chloroplast (5), is also unable to reduce PrxQ (Muthuramalingam M, Boernke F, Dietz KJ, unpublished). Focussing on the thiol regeneration systems, it is interesting that the Synechocystis PCC6803 genome encodes $\operatorname{Trx} A, \operatorname{TrxB}$, and $\operatorname{TrxQ}$, which based on sequence similarity correspond to plant $\mathrm{m}-, \mathrm{x}-$, and $\mathrm{y}$-type Trx. Both Synechocystis PCC6803 PrxQ1 and PrxQ2 are reduced by TrxA and TrxB (132), but with significant differences in kinetic characteristics. PrxQ1 has a $K_{M}$ for TrxB of $2.6 \pm 0.2 \mu M$, a $K_{M}$ of $6.6 \pm 3 \mu \mathrm{M}$ for $\operatorname{Tr} \mathrm{A}$, and the corresponding values for PrxQ2 are $17.3 \pm 2.5$ and $12.2 \pm 3 \mu M$, respectively. The study shows that cyanobacterial PrxQs are regenerated by Trx, whereas cyanobacterial GrxA and GrxB and glutathione are ineffective (132). Taken together, plant and cyanobacterial PrxQs with their negative midpoint redox potentials are functionally linked to the thioredoxin system with differences in electron transfer efficiency in vitro.

PrxQ and 2-CysPrx can also be trapped on a column loaded with the St-CDSP32 mutant C216S where the resolving Cys of the CDSP32 Trx domain is modified to serine resulting in covalent binding of potential target proteins (146). This in vitro approach shows the occurrence of heterodimers between
CDSP32 and PrxQ as well as CDSP and 2-CysPrx, respectively, which was confirmed ex vivo by coimmunoprecipitation of CDSP32 with 2-CysPrx from control and wilted plants and with PrxQ from wilted plants (146). CDSP32 also donates electrons to PrxQ in an $\mathrm{H}_{2} \mathrm{O}_{2}$ reduction assay. However, the significance of this finding remains to be shown since the concentrations used in this assay (500 $\mu \mathrm{M}$ DTT, $40 \mu \mathrm{M}$ CDSP32, and 2.5 $\mu \mathrm{M}$ PrxQ) were high and the activities with about $5 \mathrm{~mol} \mathrm{H} \mathrm{O}_{2}$ mol CDSP32 $2^{-1} \mathrm{~min}^{-1}$ low; in addition, PrxQ is associated with thylakoid membrane or lumen as discussed next. Thorough kinetic and structural analyses of a bacterial PrxQ from the phytopathogenic bacterium Xylella fastidiosa also revealed high second-order rate constants for peroxinitrite and $\mathrm{H}_{2} \mathrm{O}_{2}$ in the order of $10^{6}-10^{7} 1 \mathrm{~mol}^{-1} \mathrm{~s}^{-1}$ and significant structural reorganization during the catalytic cycle of PrxQ (63).

Higher plant PrxQ is associated with the thylakoids (88, $137,149)$. It still is an open question whether the thylakoid association occurs from the stromal or lumen side. A thorough investigation of subchloroplast distribution assigns At-PrxQ to the chloroplast lumen (137). Washed thylakoid membranes contain the major fraction of chloroplast PrxQ, which in this study is only lost from the membrane fraction upon lysis of thylakoids by passage through a Yeda press. The PrxQ protein of intact thylakoids is resistant to thermolysin treatment, but digested after addition of detergent (137). However, also in this study, part of Prx $Q$ was detected in the stromal fractions, confirming previous results where PrxQ was trapped on Trx columns with stroma protein preparations as prey $(114,146)$ or described as associated with the thylakoids from the stromal side (149) or suggested to be associated with photosystem II in the grana stacks (88). The suggested lumenal localization would assign an important function to PrxQ since it could be the only or the predominant peroxidase in the thylakoid lumen to protect lumen exposed proteins from peroxide-induced damage. On the other hand the lumenal localization poses the question as to the reductive regeneration of oxidized PrxQ during the catalytic cycle in the lumen. Reducing equivalents need to be transferred from the stroma to the lumen. One candidate system was described in context of Cytf/ $\mathrm{b}_{6}$ assembly and involves high chlorophyll fluorescence 164, which accepts electrons from stromal Trx-m and transfers these reducing equivalents to the lumenal PSI-N subunit of photosystem I (113). Recently, an additional component of the thylakoid membrane named CcdA (cytochrome c defective A, a thylakoid protein proposed to transfer electrons) was suggested to mediate the transfer of redox equivalents from stromal Trx to high chlorophyll fluorescence 164 (112). In another proteomics study of luminal proteins, PrxQ was neither identified in A. thaliana nor in spinach (157). In addition, the transit sequence of PrxQ does not bear motifs such as the twin-arginine motif for import via the Tat pathway. Immunocytochemistry gave results supporting a localization on the stromal side of the thylakoids $(88,149)$. Thus, the exact location of $\operatorname{PrxQ}$ at the thylakoid membrane still awaits confirmation.

A. thaliana plants deficient in PrxQ phenotypically develop like wild type. However, prxQ knock down lines reveal a slightly decreased oxidative stress sensitivity, modified stoichiometry of photosynthetic complexes, altered transcript levels for proteins involved in maintaining the redox homeostasis and antioxidant defense (88), and slightly increased 
oxygen evolving capacity (137). The localization and the knock-out phenotype indicate a function of plant PrxQ in photosynthesis.

\section{1-Cysteine Peroxiredoxin}

Plants and cyanobacteria express members of the typical 1CysPrx. The genomes of Synechocystis PCC6803 and Synechococcus PCC7942 encode one 1-CysPrx (slr1198 and gene 915, respectively). Synechocystis PCC6803 1-CysPrx has two additional Cys at unusual positions whose function is unknown. The cyanobacterial protein forms dimers in the presence of $\mathrm{H}_{2} \mathrm{O}_{2}$, is slowly reduced by E. coli TrxA, lacks peroxidase activity in the presence of glutathione (GSH) and Grx, and also has no phospholipase activity. This is in contrast to the human 1-CysPrx HsPrdx6, which is a bifunctional protein with both peroxidase and phospholipase activity $(28$, 99). The molecular structure of Mycobacterium tuberculosis AhpE, a 1-CysPrx, was resolved in the reduced and oxidized state. The AhpE protein conformation changes in dependence on the redox state of the catalytic Cys as seen by alterations in tryptophan fluorescence (65). Insertional inactivation of slr1198 inhibits growth of Synechocystis PCC6803 both under control and oxidative stress conditions (64). Its function in cyanobacteria essentially is not understood. The first mRNAsequence of a higher plant 1-CysPrx was cloned and sequenced from Brome grass (Bromus secalinas) in an attempt to identify genes that are expressed in hydrated dormant embryos (50). However, a biochemical function was not assigned at that time. Transcript abundance of this gene named pBS128 increases upon abscisic acid (ABA) application and simultaneously ABA arrests germination. This coincidence led to the hypothesis that pBS128 fulfils a dormancy-related function. Likewise barley late embryogenesis abundant proteins were cloned by Aalen et al. (1), among which the clone B15C revealed amino acid sequence similarity to pBS128. Stacy et al. (166) could lateron classify both genes pBS128 from Brome grass and B15C from barley as 1-CysPrx. Recombinant expression of At-1-CysPrx decreases sensitivity of E. coli cells to cumene hydroperoxide (55). Green fluorescent proteinfusions with At-1-CysPrx are targeted to the nucleus as well as the cytosol. Expression of Hv1-CysPrx is observed in the late stage of seed development and is mostly confined to the aleurone layer and the embryo of barley grains (166) and $A$. thaliana seeds (54). It should be noted that At-1-CysPrx promoter-driven $\beta$-glucuronidase activity indicates expression in vegetative tissues such as cotyledons, hypocotyls, organ abscission, and stem branching zones and at the top end of the filament at the stage of pollination (53). At-1-CysPrx expression is unaltered in ABA-deficient mutants and lowered in ABA insensitive mutant abi3-1. This dependency again links 1-CysPrx function to ABA signaling. ABA is important in maintaining seeds in the dormant state. Seeds from RNAilines with decreased levels of At-1-CysPrx germinate earlier than wild-type seeds under stress imposed by adding $200 \mathrm{mM}$ mannitol. On the contrary, $A$. thaliana plants overexpressing At1-CysPrx germinate less efficiently than wild type in the presence of osmotic, salt, and oxidative stress. Based on these findings, the authors propose the hypothesis that At1-CysPrx senses stressful conditions and is involved in delaying germination and seedling establishment in an unsuitable or suboptimal environment (55).
Transgenic tobacco overexpressing the rice 1-CysPrx (R1CPrx) develops fewer lesions upon infiltration of $5 \mathrm{mM} \mathrm{H}_{2} \mathrm{O}_{2}$ (91). Typical 1-CysPrx (B-type Prx) do not possess a second Cys that could react with the sulfenic acid derivative of $\mathrm{Cys}_{\mathrm{P}}$ once it has reacted with the peroxide substrate. Manevich et al. (98) and Ralat et al. (143) obtained evidence for the involvement of a $\pi$-type glutathione-S-transferase ( $\pi$-GST) and Grx in the regenerative catalytic cycle of human isoform Hs-Prdx6. The results suggest that 1-CysPrx in the sulfenic acid form heterodimerizes with $\pi$-GST. In the presence of GSH, the heterocomplexed 1-CysPrx is glutathionylated catalyzed by $\pi$-GST. In the last step, the thiol form of 1-CysPrx is regenerated by reaction with reduced Grx. Monteiro et al. (109) identified a novel additional pathway for reduction of 1CysPrx, which involves ascorbic acid. Ascorbate at $10 \mathrm{mM}$ concentration protects glutamine synthetase from oxidative inactivation in a mixed function oxidation assay containing 1CysPrx, $\mathrm{Fe}$, and $\mathrm{O}_{2}$. A comparison of seven 1-CysPrx proteins from different organisms, including At1-CysPrx, revealed $93 \%$ peroxidase activity at $500 \mu \mathrm{M}$ ascorbate compared to the same concentration of dithiothreitol (set to 100\%) (109). The $\mathrm{K}_{\mathrm{M}}$ for ascorbate with $\mathrm{H}_{2} \mathrm{O}_{2}$ of human Prdx6 is $760 \mu \mathrm{M}$. The glutathione-dependent catalytic efficiency $\mathrm{kcat} / \mathrm{K}_{\mathrm{M}}$ is estimated to be $3 \times 10^{6} \mathrm{l} \mathrm{mol}^{-1} \mathrm{~s}^{-1}$ and that for ascorbatedependent $\mathrm{H}_{2} \mathrm{O}_{2}$ reduction $0.9 \times 10^{6} 1 \mathrm{~mol}^{-1} \mathrm{~s}^{-1}$ and thus in the range of the thiol-dependent enzyme catalysis (99). Metabolizing and particularly photosynthesizing plant cells contain ascorbic acid at millimolar concentrations. The ascorbate concentration and reduction state decrease during ageing and under environmental stresses (47). Apparently, ascorbate is a likely candidate to reduce the sulfenic acid form of 1-CysPrx in plants. Through this connection, the hypothesis on the stress-sensing function of seed 1-CysPrx of the Aalengroup (55) might be linked to the ascorbate level and its reduction state in seeds, which both decrease along with seed viability $(38,141)$

\section{Type II Peroxiredoxins, the Most Widely Distributed Atypical 2-CysPrxs in Plants}

\section{A. Principal features of type // Prx}

The plant PrxII (atypical 2-CysPrx, type II Prx, and D-type Prx) with its two catalytic residues $\mathrm{Cys}_{\mathrm{P}}$ and $\mathrm{Cys}_{\mathrm{R}}$ spaced by 24 amino acids was independently identified by several groups in 1999. Choi et al. (31) isolated a cDNA from Chinese cabbage in a random sequencing project in a search for genes specifically expressed in flower buds. The encoded product CPrxII of 162 amino acids had a similar sequence as yeast PrxII pointing to homologous function (90). Recombinant CPrxII decomposes $\mathrm{H}_{2} \mathrm{O}_{2}$ and protects glutamine synthetase from oxidative inactivation about 6-times more efficient than Chinese cabbage 2-CysPrx (31). The higher turnover number of AtPrxII B, C, E, and F as compared to 2-CysPrx was also observed by Horling et al. $(61,62)$, however, using the artificial electron donor dithiothreitol. Verdoucq et al. (178) complemented the $\Delta$ trx yeast strain EMY63 with a plant thioredoxin variant where the resolving Cys was mutated to Ser (At-Trx3-C35S). Like for the in vitro thiol trapping system used in redox proteomics described above, this mutant strain allowed for covalent trapping of Trx targets in vivo. The authors isolated a protein complex consisting of At-Trx3 and the yeast gene product YLR109, which proved to have thiol 
peroxidase activity. YLR109 and the concomitantly identified and structurally related $A$. thaliana gene products At1g65970 or At1g60740 revealed intramolecular disulfide bond formation after reacting with the peroxide substrate and were assigned to the new subgroup of atypical 2-CysPrx, the type II Prx. In addition, Verdoucq et al. (178) showed that Trx regenerates oxidized type II Prx. Subsequently, Rouhier et al. (151) isolated a plant type II Prx from a cDNA library synthesized from poplar vascular tissue mRNA. With 162 amino acids and its peculiar arrangement of its catalytic cysteinyl residues spaced by 24 amino acids, this Pt-Prx was analyzed in detail as plant type II Prx distinct from 1-CysPrx, typical 2CysPrx, and PrxQ (151). Pt-PrxII reduces $\mathrm{H}_{2} \mathrm{O}_{2}$, t-BOOH, and cumene hydroperoxide $(\mathrm{COOH})$ and is regenerated by both thioredoxin and glutaredoxin. C51 is the peroxidatic residue indispensible for peroxide reduction, whereas the exchange of C76S reduces peroxidase activity to $25 \%$ but does not abolish it (150). From this finding it is concluded that type II Prxs can function as atypical 1-CysPrx. Glutaredoxin GrxC4 regenerates oxidized Pt-PrxII either in a dithiol or monothiol mechanism. Type II Prx are found in various phylogenetically distant groups; for example, among the six human Prx, only Prdx 5 belongs to the group of type II Prx with an additional Cys at position 152. Prdx5 is regenerated by thioredoxin (175). Structure analysis of PrxII crystals by x-ray crystallography at 1.6 ̊ and in solution by nuclear magnetic resonance spectroscopy showed that also in the reduced state the structural and catalytic unit is the dimer. However, in contrast to the dimer interface of typical 2-CysPrx, which is oriented in parallel to the planes of the central $\beta$-sheets, the interface of PtPrxII is perpendicular to the planes of the $\beta$-sheets (45) (see above: Fig. 1B). It is noteworthy that the crystals were grown in the absence of reductant in this study and, nevertheless, contained the $\mathrm{Cys}_{\mathrm{P}}$ and $\mathrm{Cys}_{\mathrm{R}}$ in their reduced state showing a low inclination to autoxidation (45). The interface is composed of amino acid residues that are largely conserved among type II Prx (D-type Prx) and involves salt bridges and hydrophobic interactions. The monomer-monomer interface of PrxII resembles the dimer-dimer interface of typical 2-CysPrx (A-type Prx) (45). In terms of contact area, the type II Prx interaction site covers about $800 \AA^{2}$, the monomer-monomer interface of typical 2-CysPrx 1050-2100 $\AA^{2}$, whereas the dimer-dimer interface of 2-CysPrx is restricted to 600-650 $\AA^{2}$ (45). As a consequence, the order of stability among the interactions decreases in the order of [monomer-monomer of typical 2-CysPrx] $>$ [monomer-monomer of PrxII] $>$ [dimerdimer of typical 2-CysPrx].

The regeneration mechanism of PrxII involves a glutathionylation step of the catalytic thiol after adopting its sulfenic acid state. Subsequently, a monothiol or dithiolglutaredoxin, respectively, accepts the glutathione and the PrxII is released in its reduced state (Fig. 10). The mixed disulfide between Grx and glutathione Grx-S-SG is reduced by two pathways (Fig. 10) where a second glutathione attacks the mixed disulfide and an oxidized form of glutathione is produced (monothiol) (154). Alternatively, a second Cys within the Grx (dithiol) acts as resolving thiol, an intramolecular disulfide bridge is formed, and GSH released. The disulfide Grx is converted to the active dithiol state by either two molecules of glutathione or another reductant, for example, ferredoxin-dependent thioredoxin reductase. In addition to kinetic parameters the midpoint redox $\mathrm{E}_{\mathrm{M}}{ }^{\mathrm{o}}$ of the dithiol/
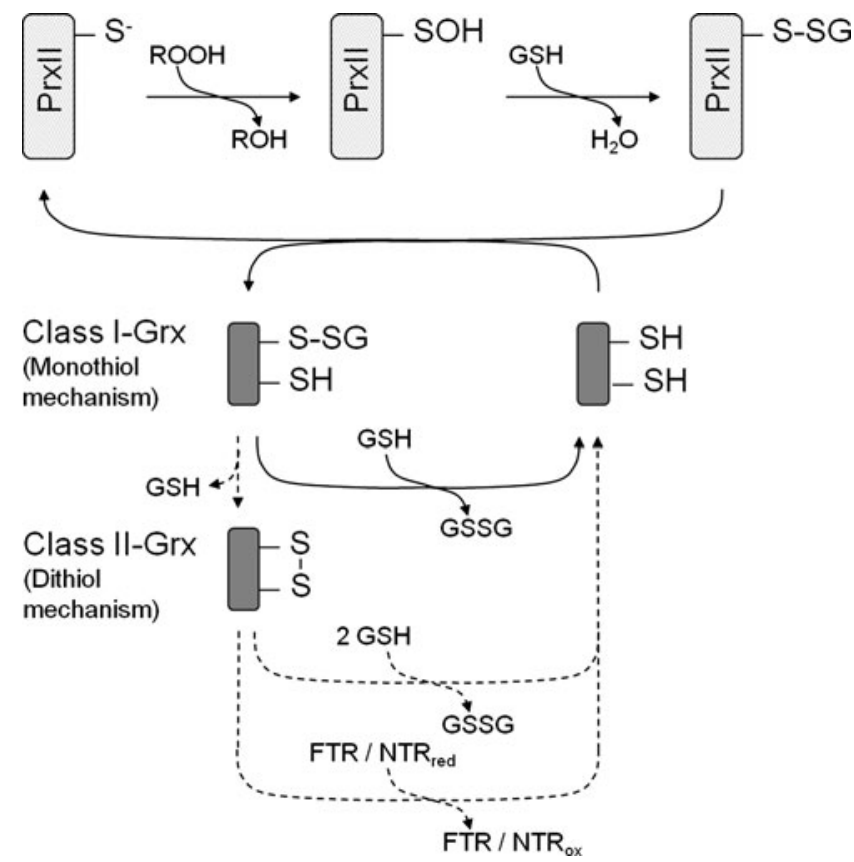

FIG. 10. Grx-dependent regeneration mechanism of type II Prx. The sulfenic acid derivative of $\mathrm{Cys}_{\mathrm{P}}$ reacts with glutathione to the S-glutathionylated Prx [cf. ref. (154)]. Grx of the monothiol type regenerates the reduced PrxII-protein by accepting the glutathione, and itself is regenerated by reduced glutathione under release of GSSG. Dithiol Grx also accepts the glutathione from PrxII-SSG to release PrxII-S ${ }^{-}$. The glutathionylated dithiol Grx releases the GSH and forms an intramolecular disulfide bridge with $\mathrm{Cys}_{\mathrm{R}}$. A thiol donor regenerates the reduced dithiol Grx. GSSG, oxidized form of glutathione.

disulfide transition provides a thermodynamic characteristics on redox linkages. Less negative midpoint potentials often are taken as indicator for a linkage to the glutathione system, for example, through Grx, and more negative ones may indicate coupling to the thioredoxin system, which in the illuminated chloroplast, is linked to the photosynthetic electron transport via ferredoxin (39). Recent in vivo imaging of the redox environment in plant cell compartments has challenged this simplified view. The redox sensor ro-green fluorescent protein, which senses the glutathione redox state catalyzed via Grx, revealed a highly negative redox state of the glutathione system of around $-310 \mathrm{mV}$ in the unperturbed resting state (104). This indicates that the glutathione system can efficiently drive redox reactions under nonstress conditions, including reductive regeneration of Prx. Furtheron, the NADPHdependent Trx system supports glutathione reduction (102), some proteins accept electrons from both Grx and Trx, which would enable slow redox equilibration between both systems $(48,151)$. There might exist more bidirectional electron flow among different Trx- and Grx-isoforms such as that observed between HvTrxh1 and HvTrxh2 (97). These observations suggest that the redox states of the distinct Grx and Trx branches in the thiol-disulfide redox network potentially equilibrate by multiple reactions, however, with distinct kinetic constraints.

Prxs interact with RNS in two ways. Lindermayr et al. (93) treated cell culture extracts with nitrosoglutathione (GSNO) 
or GSH and applied the biotin switch method to identify formerly nitrosylated proteins. In the S-nitrosylated proteome they detected PrxIIB (At1g65980), mitochondrial PrxIIF (At3g06050), and Gpx6 (At4g11600). In parallel, in NO-flushed leaves they identified chloroplast PrxIIE (At3g52960). Likewise, Romero-Puertas et al. (147) monitored the S-nitrosylated proteome of $A$. thaliana during hypersensitive response and also identified PrxIIE. Peroxynitrite $\left(\mathrm{ONOO}^{-}\right)$reduction activity was already reported for recombinant plant 2-CysPrx produced in E. coli (155), confirming previous results with 2-CysPrx from Salmonella typhimurium and Mycobacterium tuberculosis (24). At2-CysPrx complements the ROS and RNS-hypersensitive phenotype of the $S$. cerevisiae tsa1 $\Delta t s a 2 \Delta$ double mutant. Heterologous expression of cyanobacterial 2-CysPrx and PrxQ-B from Synechococcus elongatus PCC7942 in E. coli enhances cell survival in presence of the $\mathrm{ONOO}^{-}$-donor morpholinosydnonimine (SIN-1) (169). Synechocystis PCC6803 expresses a PrxII type Prx (sll1621). Knock out strains carrying an antibiotic resistance cassette in sll1621 after homologous recombination scarcely grow at moderate light intensities of $50 \mu \mathrm{mol}$ quanta $\mathrm{m}^{-2} \mathrm{~s}^{-1}$ (64). A role of PrxII in light acclimation of Synechocystis PCC6803 is indicated by the upregulation of sll1621 transcript after transferring the cells to high light, but also after adding $\mathrm{H}_{2} \mathrm{O}_{2}$ or methylviologen (170). Interestingly, Synechocystis PrxII efficiently works with glutathione as electron donor with little stimulation after addition of Grx (64).

\section{B. Cytosolic Prxll}

The Arabidopsis genome encodes four cytosolic PrxII-type peroxiredoxins. AtPrxIIB, C, and D each are 162 amino acids long and exhibit highly similar amino acid sequences with 93\%-99\% identity. The polypeptide length is conserved among species (151). The gene model for AtPrxIIA (At1g65990.1) codes for a spliced transcript of 1662 bases, a protein of 552 amino acids with a predicted molecular mass of $62.65 \mathrm{kDa}$. This protein has a high similarity to PrxIIB/C/D over the first 88 amino acids. The $\mathrm{C}$-terminal part resembles F-box proteins. A similar coding region is present in Arabidopsis lyrata with $71.2 \%$ identity, $78.9 \%$ similarity, and $6.6 \%$ gaps using EMBOSS Pairwise Alignment Algorithms. Clear evidence for the expression of PrxIIA is missing. Indirect hints are available from Western blot analysis using an antibody against PrxIIC. This antiserum should recognize PrxIIA due to its high similarity to PrxIIB/C/D in the N-terminus and labeled a band of $68 \mathrm{kDa}$ in pea. Verification of the nature of this band is missing (51).

Peroxidative activity of cytosolic type II Prx was first examined in poplar by Rouhier et al. (151). In this seminal study it is shown that peroxide reduction of cytosolic PtPrxII is maintained by regeneration systems either comprised of cytosolic Chlamydomonas reinhardtii $\operatorname{Trxh} / A$. thaliana thioredoxin reductase/NADPH or Grx/GSH/glutathione reductase. Sitedirected mutagenesis identified Cys51 as peroxidatic $\mathrm{Cys}_{\mathrm{P}}$ (150). This study also revealed that mixed disulfide formation generates a heterodimer between PtPrxII and Grx as intermediate of the catalytic cycle. Bréhélin et al. (20) localized AtPrxII B in the cytoplasm by protoplast fractionation as predicted based on the absence of signal and transit peptides. They also showed a tendency of AtPrxII B to dimerize and oligomerize when separated by SDS-PAGE in the absence of $\beta$-mercaptoethanol.

\section{Plastid PrxllE}

PrxIIE resides in the chloroplast stroma and in contrast to 2CysPrx (86) and PrxQ (88) is not associated with the thylakoids (20). Recombinant poplar PrxIIE lacking the transit peptide reduces $\mathrm{H}_{2} \mathrm{O}_{2}$ and $\mathrm{tBOOH}$ and is regenerated by Grx and Trx, most efficiently by chloroplast GrxS12 (48). PrxIIE reacts with peroxynitrate to the S-nitrosylated derivative. In vitro nitrosoglutathione and diethylamine-NONOate (DEANONOate) treatments, subsequent trypsination of the treated protein, and MS/MS analysis identified Cys121 as target of Snitrosylation. Nitrosylated PrxIIE is catalytically inactive, but its activity is readily restored by reducing agents (148). Furtheron, PrxIIE detoxifies $\mathrm{ONOO}^{-}$to produce nitrite and water. The concentration of AtPrxII E protein is about 100-fold lower than that of At2-CysPrx (130). The lower concentration may be compensated by the higher catalytic turnover and easier regeneration compared to 2-CysPrx. Dietz et al. (42) suggested that PrxII E functions as soluble thiol-dependent peroxidase next to s-ascorbate peroxidase (Apx) in the stroma and quenches peroxides released from the thylakoids.

\section{Mitochondrial PrxlIF}

The plant mitochondrion contains the type II Prx named PrxII F, which was first identified in an exhaustive search for Prx genes of the A. thaliana genome (61). Rouhier et al. (153) performed a proteomic screen with the mutagenized and immobilized Pt-Grx variant Cys30Ser. After incubation of the chromatographic material with $A$. thaliana protein extracts under oxidizing conditions, covalently bound proteins were eluted by adding reductant and analyzed by mass spectrometry. Among the set of 94 bound and identified proteins were 5 Prxs, namely, At-2-Cys-Prx, At-PrxQ, At-PrxII B, AtPrxII E, and At-PrxII F (153). To validate the interaction, the authors characterized the catalytic interaction between PrxII F and Grx. PrxII F decomposes peroxides in the presence of glutaredoxins as reductant $(46,49,153)$. Peroxidative turnover numbers are highest with $\mathrm{tBOOH}\left(30.5 \mathrm{~min}^{-1}\right)$ and similar for $\mathrm{COOH}\left(23.4 \mathrm{~min}^{-1}\right)$ and $\mathrm{H}_{2} \mathrm{O}_{2}\left(22.8 \mathrm{~min}^{-1}\right)$, and correspondingly the $\mathrm{K}_{\mathrm{M}}$ values increase from $16 \mu \mathrm{M}$ for $\mathrm{tBOOH}$ to $71 \mu \mathrm{M}$ for $\mathrm{H}_{2} \mathrm{O}_{2}$ and $326 \mu \mathrm{M}$ for $\mathrm{COOH}$ (49). AtPrxII $\mathrm{F}$ also reveals some activity with glutathione (45). PrxII F from Pisum sativum (PsPrxII F) (13) is active as peroxidase in presence of the mitochondrial Trx-o. Both redox partners PsPrxII $F$ and PsTrx-o form mixed complexes in dependence of the redox state. Thereby, an ultratight binding between PSPrxII F and PsTrx-o is established with binding constants similar to antibody-antigen interactions (13). In addition, the PsPrxII F crystallizes as hexamer (15). Thus, there is evidence that mitochondrial PrxII F undergoes dynamic conformational and structural rearrangements and redox-dependent association with interacting proteins. The tentative catalytic and rearrangement cycle of PrxII F is depicted in Figure 11. The cycle may ease the reductive regeneration of PrxII F in the stable PrxII F/Trx-o interaction and, by switching to the disassembly cycle in triggering ROS signaling from the mitochondrion. In line with this hypothesis was the result that addition of heterocomplex to a peroxidase assay gave rise to higher detoxification rates than those determined in samples where the 


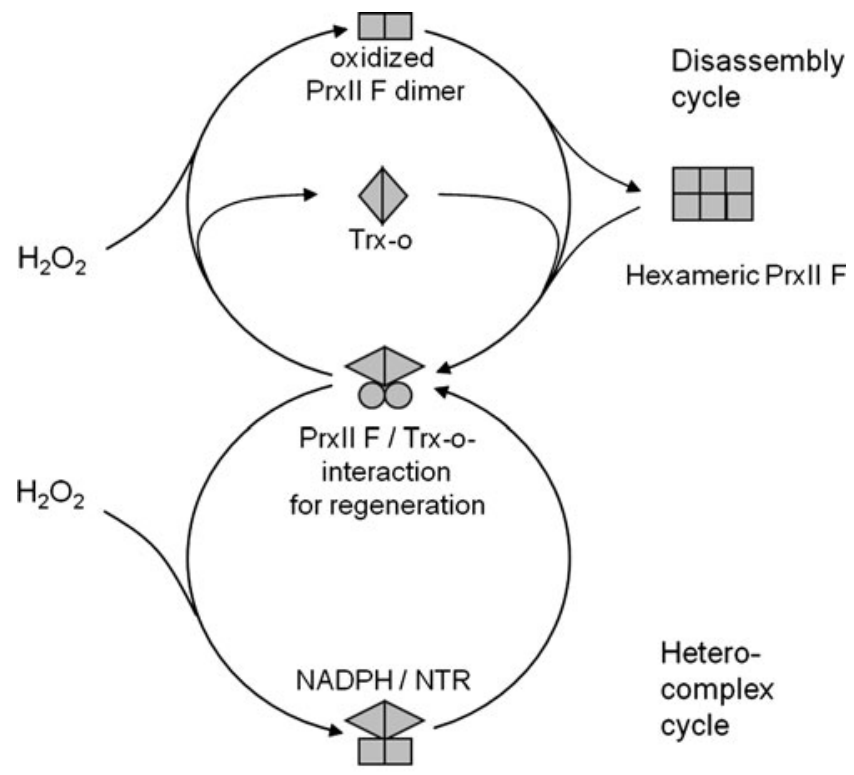

Prxll F/Trx-o-complex

FIG. 11. Hypothetical interaction cycles for PrxII F of Pisum sativum. Barranco-Medina et al. $(13,15)$ obtained evidence that Ps-PrxII F tightly interacts with Trx-o to form heterocomplexes and that Ps-PrxII F also can form hexamers. The hypothetical schematic suggests the existence of two cycles, the disassembly cycle with lower catalytic activity and the propensity to form hexamers, and the catalytically more efficient heterocomplex cycle.

partners were added separately (13). The experimental data for PsPrxII $F$ and the derived putative cycle suggest that atypical 2-CysPrxs are implicated in dynamic interaction networks and signaling. Alternatively, the existence of two cycles might be involved in regenerating eventually hyperoxidized PrxII F. In animals, Noh et al. (125) described targeting of sulfiredoxins to the mitochondrion to regenerate hyperoxidized 2-CysPrx Prdx3. Hyperoxidized AtPrxII F also occurs in plants (66). Plant sulfiredoxin shows dual targeting to both plastids and mitochondria and retroreduced hyperoxidized PrxII F (67a).

\section{E. Cyanobacterial type II Prx}

Most of the work on Prx function in cyanobacteria has been performed with the genetic model organism Synechocystis PCC6803 since its genome was completely sequenced as early as in 1996 (76). The Synechocystis PCC6803 genome encodes a single type II Prx of $21.2 \mathrm{kDa}$ (sll1621) whose transcript accumulates more than 26-fold upon exposure to methylviologen in high light compared to control (83). After insertional inactivation of the prxII gene the mutant Synechocystis PCC6803 cells develop a high light-sensitive phenotype and stop growing $10 \mathrm{~min}$ after increasing the light intensity to $200 \mu \mathrm{mol}$ quanta $\mathrm{m}^{-2} \mathrm{~s}^{-1}$ (83). In another study, the PrxIIdeficient mutant revealed severe growth inhibition even at $50 \mu \mathrm{mol}$ quanta $\mathrm{m}^{-2} \mathrm{~s}^{-1}$ (64). In wild-type Synechocystis PCC6803, the PrxII protein is estimated to amount to about $0.6 \%$ of cell protein. Recombinant sll1612-protein reduces peroxides in the substrate order of $\mathrm{tBOOH}>\mathrm{H}_{2} \mathrm{O}_{2}>>$ cumene hydroperoxide. Glutathione as reductant maintains the catalytic cycle with slide stimulation in the presence of
Grx. Reported turnover numbers are $49.5 \mathrm{~mol}$ tBOOH'mol PrxII ${ }^{-1} \mathrm{~min}^{-1}$ in the presence of $0.72 \mathrm{mM} \mathrm{GSH}$, and $55.8 \mathrm{~mol}$ tBOOH'mol PrxII ${ }^{-1} \mathrm{~min}^{-1}$ in the presence of GSH plus $0.26 \mu M$ Grx, respectively (64). In that work the Trx system was unable to support peroxide reduction, whereas data of a later study reveal that in an order of decreasing efficiency TrxQ $>$ TrxA $>$ TrxB are able to reduce PrxII (132). In fact the system consisting of PrxQ and TrxQ decomposes $\mathrm{H}_{2} \mathrm{O}_{2}$ with the highest catalytic efficiency observed in the study. The reported $\mathrm{k}_{\mathrm{cat}} / \mathrm{K}_{\mathrm{M}}$ is $8.5 \times 10^{4} 1 \mathrm{~mol}^{-1} \mathrm{~s}^{-1}$ (132). Since typeII Prxs are missing from other cyanobacteria such as Synechococcus elongates PCC7942, the essential function of sll1612-PrxII in, for example, light acclimation of Synechocystis PCC6803 must have been taken over by other Prx or proteins. The most likely candidates are members of the PrxQ-like family, which are often present redundantly $(169,170)$.

\section{Plant Glutathione Peroxidases}

Recently, plant glutathione peroxidase-like proteins (Gpx) were suggested to present a fifth class of Prx (120). Plant Gpx in comparison to their human homologs contain a cysteine instead of seleniumcysteine in their active site and exhibit a reaction mechanism similar to Prxs. Gpx reduce a broad range of hydroperoxides. During the catalytic cycle, they form an intramolecular disulfide bridge and are regenerated by Trxs. The Gpx family is encoded by eight genes in A. thaliana. All Gpx proteins share high amino acid sequence similarity (identities $>70 \%$ ) and are located in different subcellular compartments. When analyzing the relationship between the members of the Prx family and the Gpx proteins the highest similarity of about $14 \%$ is observed with PrxQ. All crystallized members of both protein families show a thioredoxin fold (120). However, evolutionarily Prx and Gpx are two distinct protein families. Therefore, a detailed description of the Gpx family is omitted in this context.

\section{Posttranslational Regulation of Peroxiredoxin Activities}

\section{A. Hyperoxidation and sulfiredoxin}

Eukaryotic genomes encode sulfiredoxins (Srx) that reduce hyperoxidized 2-CysPrx in a slow reaction with ATP and reductant as cosubstrates. Thereby, the inactive sulfinic acid form of $\mathrm{Cys}_{\mathrm{P}}$ is converted to the sulfenic acid form and subsequently with reductant to the active thiol form and the 2CysPrx can act on the next peroxide substrate. The srx gene codes for a protein with an N-terminal plastid targeting sequence and the mature Srx has a molecular mass of $11.5 \mathrm{kDa}$. A Cys residue in the C-terminal region and an adenylate binding region are implicated in the catalytic mechanism. Iglesias-Baena et al. $(66,67)$ determined the catalytic efficiency $\mathrm{k}_{\text {cat }} / \mathrm{K}_{\mathrm{M}}$ of Srx for its substrate, the hyperoxidized sulfinic acid form of 2-CysPrx, with $1.4 \times 10^{2} 1 \mathrm{~mol}^{-1} \mathrm{~s}^{-1}$. The catalytic cycle with 2-CysPrx involves three steps consisting of (i) Srxphosphorylation, (ii) intermolecular conjugate formation between Prx-SOH and phosphorylated Srx, (iii) Srx release in presence of reductant yielding a sulfinic-phosphoric mixed anhydride of 2-CysPrx, (iv) release of phosphate with generation of disulfide-S-monoxide, and (v) reduction to active thiol form (74) (Fig. 12). Thus, with glutathione in the assay a $\mathrm{P}_{\mathrm{i}}$ amount equivalent to $\mathrm{Pr} x-\mathrm{SOO}^{-}$content in the assay is 


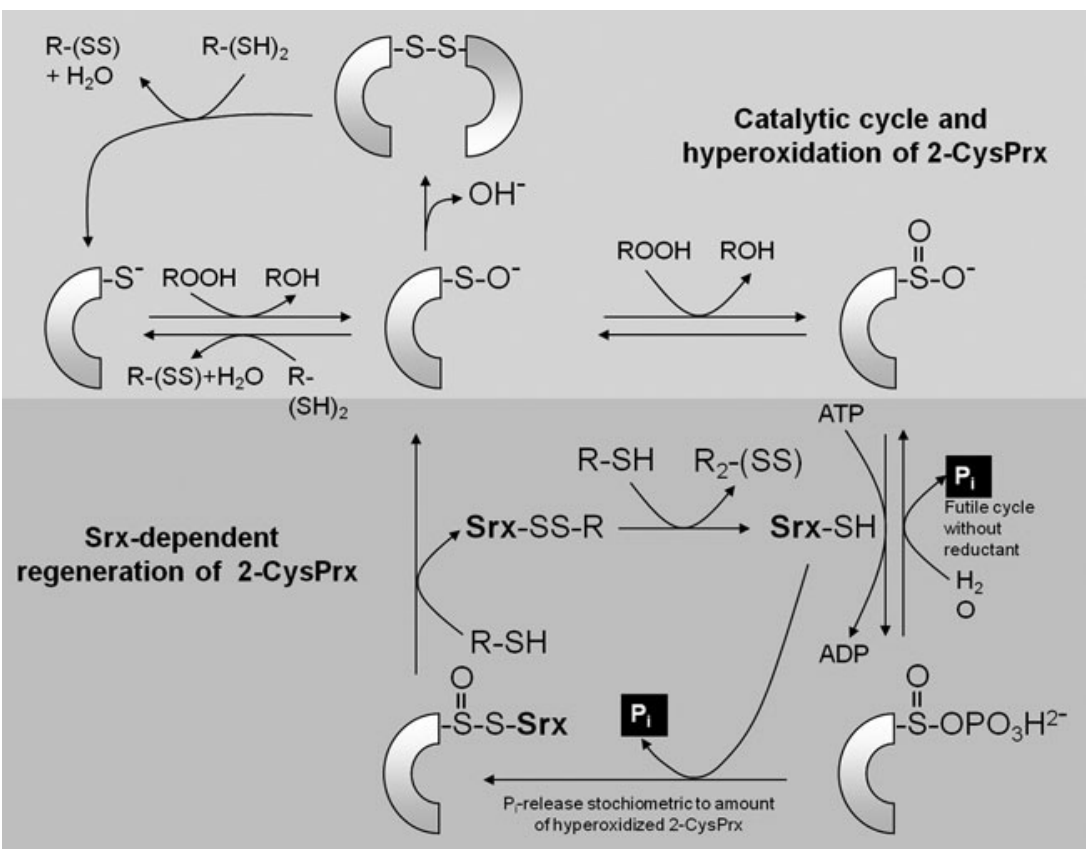

FIG. 12. Sulfiredoxin-dependent regeneration of hyperoxidized Prx. The upper part of the figure shows the catalytic cycle of Prx and its hyperoxidation. The lower part depicts the regeneration cycle converting the sulfenic acid form to the thiol form by the action of sulfiredoxins. The action of sulfiredoxins consists of several steps, which are presented in a simplified manner: (i) Phosphorylation of Srx, (ii) intermolecular complex formation of Prx-S-O-S-Srx, (iii) generation of sulfenic acid form of Prx and release of Srx, and (iv) release of reduced $\operatorname{Prx}(67)$. released from ATP. In absence of reductant, a futile cycle is established releasing inorganic phosphate from the phosphointermediate at the expense of ATP. Iglesias-Baena et al. (66) determined the activity of the futile cycle by quantifying the liberated $\mathrm{P}_{\mathrm{i}}$. Wild-type Srx releases about $0.2 \mathrm{~mol} \mathrm{P}_{\mathrm{i}} \mathrm{mol}^{-1}$ Srx $\min ^{-1}$. The authors estimate the Srx amount in chloroplast extracts to $2 \mathrm{ng} / \mu \mathrm{g}$ protein, which is in a similar range as PrxQ (88), but threefold less than 2-CysPrx in barley (87) and $A$. thaliana (130). Considering the small molecular mass of Srx with $11.5 \mathrm{kDa}$ compared to $24 \mathrm{kDa}$ for 2-CysPrx, the Srx concentration appears to be only slightly lower than the 2CysPrx concentration. Nevertheless, Srx must be considered to be a bottleneck limiting the reconvertion of 2-CysPrx-SOO ${ }^{-}$ to the catalytically active thiol peroxidase. The slow reaction rate allows for accumulation of hyperoxidized 2-CysPrx$\mathrm{SOO}^{-}$during oxidative stress. Wood et al. (182) compared the propensity to get hyperoxidized of prokaryotic and eukaryotic 2-CysPrx. Two motifs in the primary protein sequence are present in eukaryotes and correlate with the sensitivity to hyperoxidization. The authors developed the floodgate theory to link inactivation of peroxidase activity to the initiation of local cell signaling. Under normal conditions Prxs efficiently detoxify peroxides. If the cellular ROS concentration increases beyond a critical threshold, the turnover rate of reduced 2-CysPrx with peroxide increases as does the likelihood that $\mathrm{Cys}_{\mathrm{P}}-\mathrm{SO}^{-}$collides with another peroxide molecule before the $\mathrm{Cys}_{\mathrm{R}}$ reacts with $\mathrm{Cys}_{\mathrm{P}}-\mathrm{SO}^{-}$to the disulfide form $\mathrm{Cys}_{\mathrm{P}}-\mathrm{S}-\mathrm{S}-\mathrm{Cys}_{\mathrm{P}}$. As a consequence hyperoxidized 2-CysPrx accumulates, also because the regeneration system with sulfiredoxins is slow. The Prx-dependent peroxide detoxification collapses and signaling linked to ROS may propagate in the cell. Data from cocrystallization of human Prdx1 and Srx with $0.26 \mathrm{~nm}$ resolution reveal the structural basis of interaction needed for Srx-dependent retroreduction of hyperoxidized 2-CysPrx (73). Major conformational rearrangements bring the hyperoxidized catalytic center of Prx in close contact with the Srx center; the Srx molecule is sandwiched between the active site surface of one PrdxI subunit and the C-terminal tail from the adjacent PrxI subunit (73) (Fig. 13). Further in silico structure modeling suggests that the Prx/Srx interaction is still possible in oligomers; however, high-molecular-mass assemblies others than toroids are destabilized in the presence of Srx. Thus, the authors suggest cellular functions of Srx as regulatory interactor in addition to its enzymatic function in Prx retroreduction (73).

\section{B. Nitrosylation}

Nitric oxide functions as signaling molecule in animals. Tentative evidence indicates a signaling and regulatory role of NO also in plants (111). GSNO formed by reaction of nitrous acid with glutathione or enzymatically is considered as storage pool of NO. GSNO also reacts with other thiols to form nitrosothiols. S-nitrosylation can modify protein function and activity as shown, for example, for methionine adenosyl-

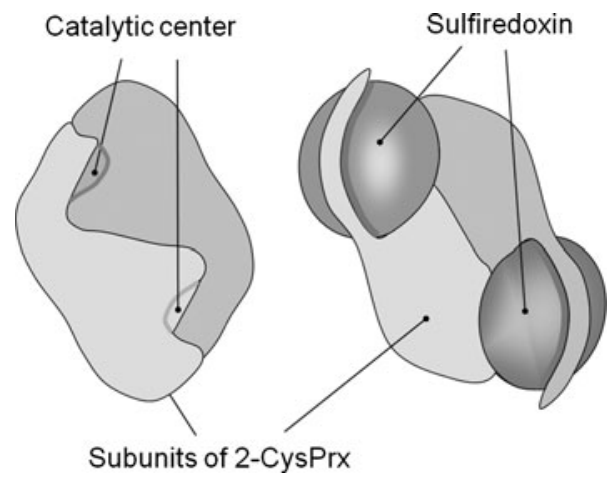

FIG. 13. Simplified scheme of the structure of the dimeric 2-CysPrx with two molecules of complexed Srx. The hyperoxidized catalytic center of Prx is indicated in the dimer on the left-hand side. On the right-hand side; the Srx molecule is sandwiched between the active site surface of one Prx subunit and the C-terminal tail from the adjacent Prx subunit [adapted from ref. (73)]. 
transferases or glyceraldehyde-3-phosphate dehydrogenase under salt stress $(94,180)$. S-nitrosylation is a controlled and rapidly reversible posttranslational modification that mediates flexible and specific responses to environmental changes (101). Lindermayr et al. (93) searched for in vitro S-nitrosylated proteins in $A$. thaliana cell culture extracts by treatment with GSNO and subsequent labelling with biotin using the biotin switch method. By mass spectrometry, they identified the glutathione peroxidase (Gpx, At4g11600), PrxII F (At3g06050), and PrxII B (At1g65980) among other targets as being nitrosylated under these conditions. Ex vivo proteomics with $A$. thaliana undergoing hypersensitive response identified S-nitrosylated PrxII E, the chloroplast located type II Prx (147). S-nitrosylation inhibits peroxidase activity of AtPrxII E. PrxII E possesses peroxynitrite reductase activity, which is inhibited by S-nitrosylation (148). Based on these interdependencies between S-nitrosylation and ROS and RNS detoxification activity, it is tempting to assume that PrxII E plays a role as switch or at least modulator in ROS- and RNSdependent cell signaling.

\section{Other posttranslational Prx modifications}

Some nonplant Prxs are subjected to posttranslational modifications apart from redox modification and S-nitrosylation of the cysteine residues. Chang et al. (27) described human Prdx1 phosphorylation by cyclin dependent kinase 2 (cdc2) in vitro and coappearance of phosphorylated Prdx1 protein in HeLa cells expressing Cdc2 (cyclin-dependent cell cycle kinase) during the cell cycle. In vitro peroxidase activity of phosphorylated Prdx1 drops to $20 \%$ of the nonphosphorylated form. Woo et al. (181) observed phosphorylation at Tyr 194 of membrane-associated human Prdx1, a typical 2-CysPrx, in response to growth factor application and during the wound healing response. Tyr194 phosphorylation inhibits peroxidase activity of Prdx1. Like hyperoxidation, this phosphorylation reaction is discussed as a mechanism to allow for local $\mathrm{H}_{2} \mathrm{O}_{2}$-dependent signaling in the cells. Likewise phosphorylation of human Prdx6, a 1-CysPrx, at Thr 177 increases the acidic and calcium-independent phospholipase 2 activity of this unique $\operatorname{Prx}$ (184). These three examples show the significance of phosphorylation in controlling Prx activity, for example, in humans. Large-scale phosphoprotein profiling in rice cell line in comparison with Medicago truncatula and A. thaliana (119) or analysis of the A. thaliana seedling phosphoproteins (145) did not identify Prxs as part of the plant phosphoproteome. Thus, at present evidence for phosphorylation-dependent regulation of plant Prxs at conventional sites (Ser, Thr, and Tyr) is missing. In plants, one report describes MgATP-dependent autophosphorylation of the resolving $\mathrm{Cys}_{\mathrm{R}} 175$ yielding [Prx-(Cys175)- $\mathrm{SO}_{2} \mathrm{PO}_{3}{ }^{2-}$ ] or [Prx(Cys175) $\left.-\mathrm{SO}_{3} \mathrm{PO}_{3}{ }^{2-}\right]$. The authors interpreted this modification as a mechanism to link the energy state of the chloroplast to the activity state of chloroplast 2-CysPrx (3). In contrast to the phosphorylation reactions described above involved in regenerating hyperoxidized 2-CysPrx by Srx and regulatory phosphorylation, this reaction did not require a catalyst like protein kinases or sulfiredoxin. The significance of the Cys175 phosphorylation awaits clarification. In the same study, supplementation with MgATP inhibited the peroxidase activity of rapeseed 2-CysPrx concomitant with aggregation to a high-molecular-mass form $>2.5 \times 10^{6} \mathrm{Da}$. The inhibition of peroxidase activity was restored after adding the $\mathrm{Mg}$-chelator ethylenediamine tetraacetic acid.

Another posttranslational modification not investigated for plant Prxs is N-terminal acetylation. Thus, it was shown for human Prdx2 that its N-terminal acetylation enhances peroxidase activity and protects $\mathrm{Cys}_{\mathrm{P}}$ against irreversible hyperoxidation (160). Glutathionylation of 2-CysPrx (At3g11630) was detected in a study aiming at defining the plant thiolation proteome of oxidatively stressed $A$. thaliana cells (44). Recovery of 2-CysPrx in this study could either be explained by glutathionylation of one of the two Cys contained in 2-CysPrx or by complexation of 2-CysPrx with another thiolated protein. The regulation of glutathionylation and dethionylation of Prx and the functional significance of this reversible posttranslational modification in plant metabolism still need to be explored.

\section{Role of Peroxiredoxins in Plant Metabolism}

The multiple sites of subcellular Prx localization point to site-specific functions in plant cells (Fig. 14). The local distribution might be explained by three scenarios. The presence at multiple sites is needed to meet the requirements for local peroxide detoxification in general antioxidant defense. To achieve this function, Prx isoforms should be present in all metabolically active and relevant compartments. However, in addition to the (at least) nine expressed Prxs, A. thaliana encodes a set of other peroxidases consisting of nine ascorbate peroxidases and seven Gpx, and also three peroxisomal catalases (108). Thus, the question is: What makes Prx-dependent peroxide detoxification unique and irreplacible by other antioxidant systems during evolution? (i) Prxs use a thiol-based catalytic mechanism, employ oxidoreductases as cosubstrates, often cover a broad peroxide spectrum as substrates, efficiently function at low peroxide concentrations and thus may play a role in general antioxidant defense. The Prx type that fits best to this scenario of general antioxidative activity are the type II Prx, which are present in cytosol, plastid, and mitochondrion. (ii) Alternatively, Prx may function in specific metabolic context of the respective compartment, for example, in photosynthesis or respiration. This scenario will be

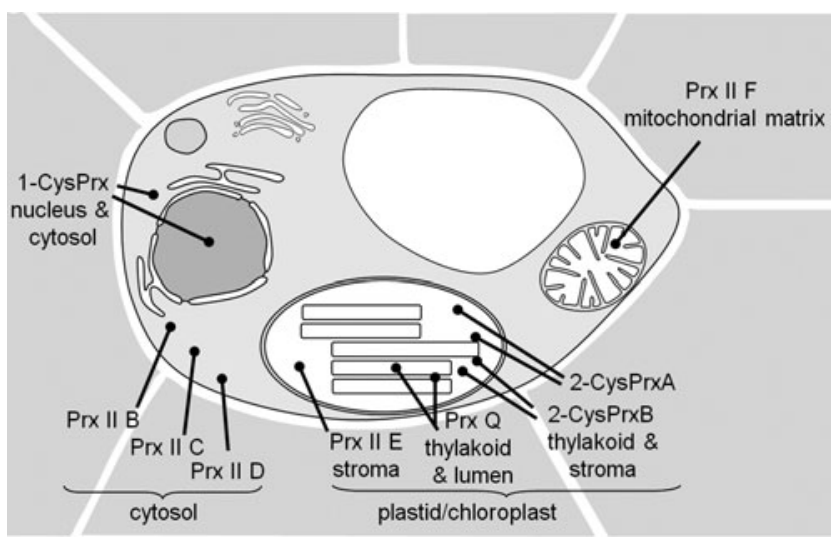

FIG. 14. Overview on the subcellular localization of $A$. thaliana Prxs. The figure depicts a plant cell with various compartments and organelles. The arrows indicate the localization of the Prx proteins encoded by the A. thaliana genome [cf. ref. (42)]. 
discussed in the following section. (iii) The third scenario assigns a function to Prx as redox sensor, redox element in signaling networks, and as protein-protein interactors. This scenario requires the presence of specific Prx in specific subcompartments. This function will be addressed in the subsequent section dealing with Prx in cell signaling.

\section{A. Plastids and photosynthesis}

Three different types of Prx reside in the plastids (42). Recently, Pitsch et al. (138) conducted a detailed genome analysis for chloroplast antioxidant enzymes in the lycophyte Selaginella moellendorffii, the liver moss Physcomitrella patens and the unicellular alga Chlamydomonas reinhardtii. The spike moss $S$. moellendorffii diverged from the fern and seed plant lineage about 400 million years ago (11).The genome-wide search identified four 2-CysPrx, four PrxQ, and two PrxII targeted to the S. moellendorffii chloroplast, two 2-CysPrx, three PrxQ, and two PrxII in $P$. patens chloroplasts, and three 2-CysPrx, one PrxQ, and one PrxII in C. reinhardtii chloroplasts. The authors conclude on partially independent evolution of these chloroplast-targeted Prxs (138). Despite some variation, the general conclusion remains valid that chloroplast as usual Prx equipment contain at least one 2-CysPrx, one $\operatorname{PrxQ}$, and one PrxII E (42).

Prx accumulate to high concentrations. At-2-CysPrxA has a value of $0.62 \%$ for relative protein abundance estimated from its spot volume in $2 \mathrm{D}$ separations and of 0.30 relative concentration as calculated by dividing the relative protein abundance by molecular mass. The values for At-2-CysPrx B are $0.61 \%$ for relative abundance and $0.28 \%$ for relative concentration. Thus, 2-CysPrxs are among the top 20 most abundant stromal proteins of $A$. thaliana with concentrations similar to phosphoglycerate kinase-1 (130). PrxIIE $\left(0.24 \times 10^{-2}\right.$ relative concentration) is only present at $0.8 \%$ normalized concentration relative to 2 -CysPrxA set $100 \%$ (130). In the context of the thiol-disulfide redox regulatory network, it should be noted that Trx-m1, $-\mathrm{x}$, and -f1 are significantly lesser concentrated than the Prxs $\left(0.10 \times 10^{-2}, 0.04 \times 10^{-2}\right.$, and $0.01 \times 10^{-2}$ relative concentration, respectively), whereas peptidyl-prolyl-cis/trans-isomerase Cyp20-3 $\left(27 \times 10^{-2}\right.$ relative concentration) is present at concentrations similar to each 2-CysPrx (130). The 2-CysPrx concentrations obtained in this proteomics study are similar to those estimated from dilutions series of chloroplast extracts and comparison of signal intensities with reference protein by Western blot analysis. By this approach König et al. (86) estimated the stromal 2-CysPrx concentration to $60 \mu \mathrm{M}$. One of the functions established for 2CysPrx in vitro is the protection of protochlorophyllide synthesis, which takes place through the stimulation of an aerobic cyclase by the NTRC / 2-CysPrx system (168). Only very small amounts of protochlorophyllide are formed in an in vitro test system in the absence of $\mathrm{H}_{2} \mathrm{O}_{2}$-scavenging systems. Addition of NTRC combined with 2-CysPrx enables chlorophyll precursor biosynthesis at rates similar to those after addition of catalase, which is absent from the chloroplasts (168). The stimulatory effect of the NTRC/2-CysPrx system suggests that $\mathrm{H}_{2} \mathrm{O}_{2}$ scavenging is important and through coupling to the NADPH-dependent NTRC system independent of lightdriven generation of reducing equivalents (165). This coupling to NADPH/NTRC may be particularly important in darkened leaves or in seedlings and young leaves before photosynthesis is established, allowing the system to synthesize precursors for chlorophyll biosynthesis and assembly of the photosynthetic apparatus. In line with this hypothesis 2-CysPrx accumulation precedes expression of other antioxidants during early $A$. thaliana seedling development (131). Further, 2-CysPrx deficiency inhibits photosynthesis and chlorophyll accumulation during plant development (8). Disturbance in chlorophyll biosynthesis in $\mathrm{Mg}$ protoporphyrin monomethylester cyclase-deficient tobacco causes upregulation of 2-CysPrx protein amount (136). The early requirement for 2-CysPrx during seedling and leaf development and the effect of 2-CysPrx deficiency on photosynthesis might be interlinked through the protection of chlorophyll biosynthesis.

2-CysPrx appears to have a high propensity to interact with other proteins. The exergonic cleavage of fructose-1,6-bisphosphate to fructose-6-phosphate and inorganic phosphate by chloroplast fructose-1,6-bisphosphatase (FBPase) is a highly regulated key step in the Calvin cycle. Due to the basically irreversible nature of the reaction, the FBPase is under tight feedback and feedforward control by metabolites and also by the thioredoxin system (30). The oxidized disulfide form is insensitive toward substrate activation (144). Reduction of the disulfide bridge to the thiol form by thioredoxin $\mathrm{f}$ thus activates, reoxidation, for example, in darkness, inactivates FBPase, thereby controlling carbon fluxes in the Calvin cycle. Caporaletti et al. (25) observed that the oxidized form of 2-CysPrx activates the rapeseed FBPase independent of Trxdependent reduction. The authors hypothesize that the regulatory interaction between 2-CysPrx as redox sensor and FBPase as one of the committed steps in the Calvin cycle allows for adjustment of Calvin cycle activity under conditions of oxidative stress.

Potato plants lacking the thioredoxin CDSP32, which is an electron donor to 2-CysPrx, are more sensitive to high light than wild type as revealed by inhibition of relative quantum yield of photosystem II (21). By quantifying lipid peroxidation products in leaf extracts or monitoring the chlorophyll thermoluminescence as marker of lipid peroxidation, CDSP32deficient mutants accumulate lipid peroxides. The authors suggest that this is due to insufficient 2-CysPrx regeneration and hyperoxidation of 2-CysPrx (24). As a consequence, lipid peroxides would not be detoxified by the 2-CysPrx system and accumulate to toxic levels causing the inhibition of photosystem II.

2-CysPrx associates with the thylakoid membrane (86). Fractionation of thylakoids into photosynthetic complexes and coimmunoprecipitation revealed the association of 2CysPrx protein with photosystem II (118). Quantum yield of photosystem II (ФPSII) as determined by chlorophyll a fluorescence analysis is lower in young 2-CysPrx-deficient plants than in wild type (8). The difference in ФPSII is enhanced in the presence of lincomycin, which inhibits organellar protein synthesis, suggesting that the 2-CysPrx-deficient mutants depend on organellar protein synthesis more than wild type. The data indicate that either 2-CysPrx protects photosystem II by localized peroxide detoxification or that the interaction affects properties of photosystem such as stability or turnover.

PrxQ represents about $0.3 \%$ of chloroplast protein (88). PrxQ is associated with the thylakoid membrane, most likely with the lumen (137) or intermembrane stacks (88). prxQknock out $A$. thaliana plants lack any conspicuous change in 
phenotype $(88,137)$. PSII content as determined from redoxdependent differential absorption spectroscopy relative to wild type is reduced by about $17-22 \%$ and the cytochrome- $b_{6} f$ content by about $6 \%-15 \%$ (88). The rate of oxygen evolution per chlorophyll is increased by about $15 \%$ in thylakoids from prxQ-knock out-plants compared to wild type (137). Without giving a clear and conclusive picture, the data indicate that PrxQ functions in immediate context of photosynthesis.

The physiological function of PrxII E as third plastid targeted Prx has not been studied in greater detail in context of photosynthesis, except for its role in peroxinitrite detoxification, S-nitrosylation, and pathogen-dependent signaling (148). Comparison of 4-week-old $A$. thaliana with modified PrxII E contents, which either were reduced to about $20 \%$ in a T-DNA insertion line or increased to $150 \%$ in overexpressing lines, and wild-type plants shows no significant differences in appearance, growth rate, chlorophyll content, and fresh weight (148).

\section{B. Mitochondria and respiration}

Plant mitochondria generate ROS during respiratory electron transport at complexes I and III, and from reduced ubiquinone (120). The uncoupling protein (UCP) and the alternative oxidase assist in minimizing $\mathrm{O}_{2}{ }^{\bullet-}$ generation under conditions of overreduction. Apx, Gpx, and PrxII F detoxify $\mathrm{H}_{2} \mathrm{O}_{2}$ released from superoxide dismutation by MnSOD. Sweetlove et al. (172) applied oxidative stress to cultured $A$. thaliana cells by adding menadione a well known superoxide generator or $\mathrm{H}_{2} \mathrm{O}_{2}$ to the suspension medium, isolated mitochondria, and explored the changes in mitochondrial proteome. PrxII F was among the top 25 proteins whose abundance increased more than threefold (172). PrxIIF is targeted to the soluble matrix of the mitochondrion $(13,46$, 49). Prx IIF is constitutively expressed in all tissues and catalyzes detoxification of various peroxides. Poplar PrxIIF in conjunction with poplar GrxC4 and in the presence of a glutathione regeneration system realizes peroxide turnover numbers of peroxide reduction ranging between 20 and $30 \mathrm{~mol}^{-(m o l}$ PrxII F min) $)^{-1}$ (49). Similar turnover numbers are reported for AtPrxII F/AtGrx-CxxS10 in the presence of saturating GSH concentrations or with AtTrx-o and AtNTRA (46). The $\mathrm{K}_{\text {cat }} / \mathrm{K}_{\mathrm{M}}$ value of poplar PrxIIF with $\mathrm{H}_{2} \mathrm{O}_{2}$ is $5.35 \times 10^{3} 1 \mathrm{~mol}^{-1} \mathrm{~s}^{-1}$ and $3.19 \times 10^{4}$ with $\mathrm{tBOOH}$. Thus, the mitochondrial PrxII F is regenerated by both the thioredoxin and the glutaredoxin system, and with lower activity by glutathione. A tight binding between PrxIIF and Trx-o occurs in the pea system with either recombinant proteins and in extracts from isolated mitochondria (13). The interaction was verified by three different methods, namely, ITC, size exclusion chromatography, and coimmunoprecipitation. Furtheron, PrxII F was crystallized and the published space coordinates show a hexameric arrangement as crystal unit in line with the chromatographic results (15). As discussed above, the assembled heterocomplex of Trx-o/PrxII F catalyzes peroxide detoxification more efficiently than the dimer and hexamer. Apparently, the pea mitochondrial Trx-o/PrxII $F$ system undergoes surprising conformational dynamics with significance for PrxII F function.

The phenotype of PrxIIF knock out lines is inconspicuous under normal growth conditions but revealed inhibited root growth under stress conditions evoked by $\mathrm{Cd}$ or salicylhy- droxamic acid administration (46). Elevated ascorbate peroxidase and glutathione peroxidase activities in the knock out lines indicate sufficient compensation of lacking PrxII F under unstressed conditions. A redox signaling function of PrxII F was proposed based on modified nuclear and mitochondrial gene expression in the knock out lines (46). Thus, under control conditions, PrxII F-knock out plants have elevated transcript levels encoding, for example, catalase, ferredoxin, PrxII B, and cyclophilin isoforms (46).

\section{Peroxiredoxins as Chaperone and in Plant Redox Signaling}

\section{A. Prx as chaperone}

2-CysPrx in the high-molecular-mass conformation adopts the function as a molecular chaperone, which protects other proteins from denaturation. This was first observed by Jang et al. (70), who described chaperone function for the yeast 2CysPrxs cPrxI and II. Oxidative and heat stresses convert the low-molecular-mass form to high-molecular-mass complexes in yeast. The conformational switch coincides with the transition from peroxidase to chaperone activity (70). The tests for chaperone activity employ heat-dependent aggregation of vertebrate citrate synthase and dithiothreitol-dependent aggregation of insulin, respectively. The aggregation of citrate synthase and insulin is suppressed in presence of hyperoxidized high-molecular-mass 2-CysPrx. These tests indicate binding of the particular 2-CysPrxs to exposed hydrophobic surfaces of denatured proteins. The same chaperone-like activity is also associated with plant 2-CysPrx as shown for rapeseed, Chinese cabbage, and $A$. thatiana using the same test assays (3, 79, 118). Kim et al. (79) analyzed recombinant Chinese cabbage 2-CysPrx and observed a switch between low- and high-molecular-weight form under oxidative stress. 2-CysPrx in the low-molecular-weight form expresses peroxidase activity, whereas high-molecular-weight forms show chaperone function. The authors suggest that the precise regulation of 2-CysPrx conformation and hence function might be involved in protecting plant chloroplasts from photo-oxidative stress. The validity of the hypothesis and the physiological significance of the chaperone function remain elusive so far.

\section{B. Prx in ROS-dependent signaling}

ROS play important roles as metabolic messengers in intracellular communication. Generation and modulation of subcellular ROS levels, ROS perception by sensors, and ROS reactions with diverse target molecules orchestrate gene expression and control gene product function (47). Thus, ROS act as crucial and ubiquitous cues in triggering cellular signaling networks and affect plant development and acclimation. Prxs are involved in ROS- and redox-dependent signaling by (i) modulating $\mathrm{H}_{2} \mathrm{O}_{2}$ concentrations, (ii) processing alkylhydroperoxides, (iii), draining electrons from redox transmitters within the thiol-disulfide redox regulatory network (Fig. 7), (iv) performing redox interactions with other proteins, (v) undergoing redox-dependent conformational changes with concomitant alterations in binding to other partners, and (vi) decomposing peroxinitrite (40). The latter mechanism will be discussed in a separate section on RNSdependent signaling. Significant roles of Prxs in ROS signaling 
of nonplant species have been proven, for example, in yeast, where the $\mathrm{H}_{2} \mathrm{O}_{2}$-dependent activation of the transcription factor Pap1 is linked to a redox signal delivered via the Prx named Tpx1 to Pap1 (179) or Tpx1 regulates the peroxide-induced Sty1 activation as part of the stress-activated protein kinase pathway (177). The universal minicircle sequence-binding protein (UMSBP) recognizes the replication origin of the kinetoplast DNA, which is the mitochondrial DNA of trypanosomatids. UMSBP activity is under redox control where tryparedoxin peroxidase, a 2-CysPrx, directly oxidizes UMSBP protein, whereas tryparedoxin reduces it. The authors suggest a redox switch mechanism regulating DNA replication and segregation (162). Some evidence suggests that Prxs might associate with DNA under certain conditions (164).

Prxs decompose $\mathrm{H}_{2} \mathrm{O}_{2}$ in parallel to the other cellular systems. As discussed above, diverse data suggest that their catalytic efficiency is in the range of ascorbate peroxidase. There exist no reliable tests to determine total Prx activity in extracts or cell fractions due to the diversity and specificity of regeneration. Thus, neither E.coli TrxA, nor specific Grx-isoforms, nor the artificial electron donor dithiothreitol reduce all Prx with equal efficiency. Being aware of these shortcomings, the chloroplast Trx-dependent Prx activity was compared to Apx activity in lysed chloroplasts using TrxA as donor and the NADPH oxidation monitored (42). The result indicates a similar capacity of the Trx-dependent and the ascorbate-dependent $\mathrm{H}_{2} \mathrm{O}_{2}$ reduction in $A$. thaliana chloroplasts. Thus, under regular conditions, Prxs appear to effectively contribute to quenching of $\mathrm{H}_{2} \mathrm{O}_{2}$. This conclusion is supported by the finding that $A$. thaliana lines deficient of both 2-CysPrx isoforms accumulate more $\mathrm{H}_{2} \mathrm{O}_{2}$ (142). At elevated ROS levels, the hyperoxidation of 2-CysPrx arrests the peroxidase activity. The floodgate theory of Wood et al. (182) proposes that the hyperoxidation-linked inactivation of 2-CysPrx enables local accumulation of peroxides to trigger cellular signaling and appropriate (genetic) responses. Hyperoxidized 2-CysPrx is detectable in leaves using antibodies against sulfinic acid Prx and has been reported in several studies $(24,66,67,133,146)$.

Some Prxs act on certain lipid peroxides (86). Lipid peroxide-derived oxylipins, including jasmonates, modulate nuclear gene expression (117). Prx activity with alkylhydroperoxides may interfere with lipid signaling by changing substrate concentrations for other reactions; likewise, changes in Prx activity may modify the effect that Prxs have on lipid signaling. Transgenic plants with low or lacking Prx activity have been studied in relation to the state of the antioxidant systems and protein carbonylation $(9,46,88,142)$ but not in relation to lipid peroxide accumulation and lipid signaling.

The thiol-disulfide redox network has been introduced above (Fig. 7) (39). Oxidized Prxs receive their electrons from specific redox transmitters. This is in contrast to $\mathrm{H}_{2} \mathrm{O}_{2}$ detoxification by ascorbate peroxidase, which directly oxidizes the low-molecular-mass antioxidant ascorbate during peroxide reduction. The redox transmitters such as NTRC, Trx-x, and CDSP32 also control the redox state of other target proteins. High rate drainage of electrons by the Prx system will effectively compete with other acceptors and cause a shift to a more oxidized state of the other targets. Starch synthesis is the example of another regulatory target of NTRC (106).

Prxs interact with other proteins and protein complexes, for example, At-2-CysPrx with fructose-1,6-bisphosphatase (25), with photosystem II (118) and chlorophyll biosynthetic com- plex (168), and PsPrxII F with PsTrx-o (13). The search for additional interactions has not been exhaustive and yeast-2hybrid screens indicate that 2-CysPrx and PrxQ as bait attracts several prey proteins (Roloff, Klein and Dietz unpublished). In addition with 2-CysPrx (At3g11630) as input into the protein-protein interaction database BAR (http://bar. utoronto.ca/) a large network emerges with 176 nodes after observation using Cytoscape $(33,41)$. However, many interactions within the predicted network unlikely occur in vivo, since the 2-CysPrx is a plastid protein while the majority of the suggested interacting proteins localize to extra-plastid compartments. The significance of known and novel interactions in regulation and signaling awaits clarification.

\section{Prx in RNS-dependent signaling}

S-nitrosylation of type II Prx was described above and is established to modify peroxidase activity. In addition to the reaction with thiols, $\mathrm{NO}$ efficiently reacts with superoxide anion $\left(\mathrm{O}_{2}{ }^{--}\right)$to produce $\mathrm{ONOO}^{-}$. In fact this reaction proceeds at an about fourfold higher rate than disproportionation by superoxide dismutase (68). If $\mathrm{NO}$ and $\mathrm{O}_{2}{ }^{--}$are formed at the same subcellular site, the product $\mathrm{ONOO}^{-}$is released. Thus, $\mathrm{ONOO}^{-}$is likely to be continuously formed at low rates in photosynthesizing chloroplasts and in stimulated amounts during plant-pathogen interactions (148). $\mathrm{ONOO}^{-}$rapidly reacts with any type of molecules, including proteins causing protein nitration. In contrast to animal cells, where $\mathrm{ONOO}^{-}$induces cell death (19), plant cells can cope with high levels of $\mathrm{ONOO}^{-}$(37). Tyrosine nitration interferes with tyrosine phosphorylation and function of Tyr phosphoproteins either by inhibiting the normal function of the proteins or by inducing structural changes similar to those imposed by protein phosphorylation (81). RomeroPuertas et al. (148) suggest that modifying the S-nitrosylation state of PrxII E may modulate NO- and ROS-mediated signaling. Thereby, PrxII E might finetune the damaging and signaling effects of $\mathrm{ONOO}^{-}$. Exposure of wild-type or prxIIE knock down lines to high $\mathrm{ONOO}^{-}$concentrations does not induce cell death, suggesting that accumulating $\mathrm{ONOO}^{-}$is not a sufficient mediator in NO-induced cell death (148). The authors suggested a model where S-nitrosylation of PrxIIE regulates the transduction of $\mathrm{NO}$ - and ROS-linked signals by fine-tuning the damaging and signaling effects of its own radicals. The function of PrxII E in RNS signaling is summarized in Figure 15. As potential signaling route RomeraPuertas et al. (148) propose the modification of Tyr residues in proteins to yield nitrotyrosine derivatives. S-nitrosylation also inhibits the $\mathrm{ONOO}^{-}$detoxification activity of PrxII E, causing a dramatic increase of $\mathrm{ONOO}^{-}$-dependent nitrotyrosine residue formation (148). The balance between ROS, NO, and $\mathrm{ONOO}^{-}$determines PrxII E activity, which in turn modulates the cell death program and protein tyrosine nitration during, for example, hypersensitive response. Protein nitration is known as selective and reversible process (84) that affects signaling processes linked to protein Tyr phosphorylation. This is achieved either by inhibiting the protein activity normally associated with the phosphorylated form or by mimicking the conformational changes triggered by protein phosphorylation (148). A similar function as proposed for PrxII E in $\mathrm{ONOO}^{-}$ signaling could also apply to 2-CysPrx, which has been shown to detoxify $\mathrm{ONOO}^{-}(155)$. 


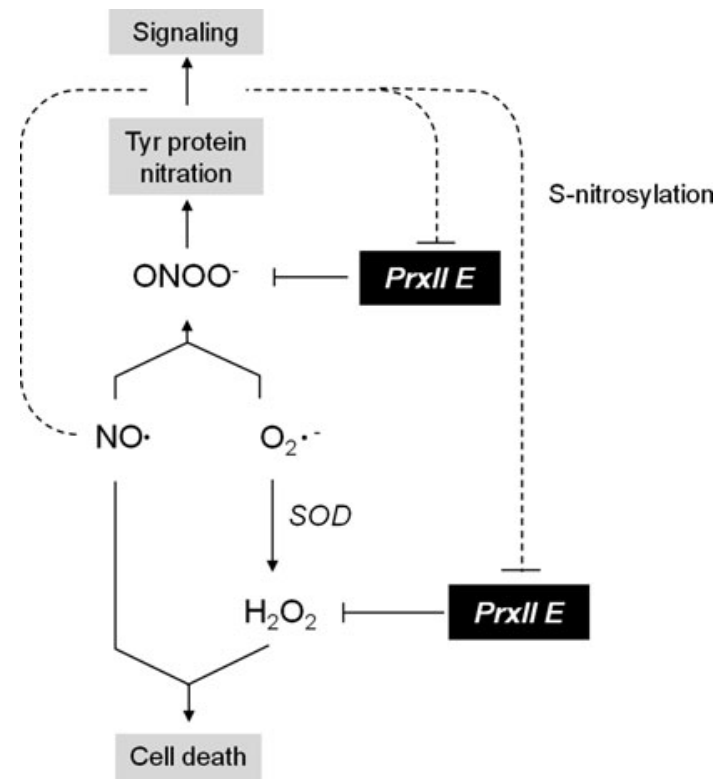

FIG. 15. Role of PRX in reactive oxygen and reactive nitrogen species signaling. $\mathrm{NO}, \mathrm{ONOO}^{-}, \mathrm{O}_{2}{ }^{--}$and $\mathrm{H}_{2} \mathrm{O}_{2}$ play multiple roles in the cell. Prxs detoxify both $\mathrm{H}_{2} \mathrm{O}_{2}$ and $\mathrm{ONOO}^{\bullet-}$, SOD dismutates $2 \mathrm{O}_{2}{ }^{\bullet-}$ to $\mathrm{H}_{2} \mathrm{O}$ and $\mathrm{H}_{2} \mathrm{O}_{2} \cdot \mathrm{O}_{2}{ }^{\bullet-}$ reacts with $\mathrm{NO}$ to form the highly reactive $\mathrm{ONOO}^{-}$. NO Snitrosylates PrxII E. Thus, if NO accumulates in the presence of $\mathrm{O}_{2}{ }^{-}$, , both $\mathrm{ONOO}^{-}$is formed and PrxII E is inhibited by S-nitrosylation. Thus, PrxII fails to detoxify the $\mathrm{ONOO}^{-}$. This enables nitration of target proteins at regulatory sites and signal transduction is initiated. This figure extends on the model presented by Romano-Puertas et al. (148).

\section{Regulation of Peroxiredoxin Gene Expression}

The specific contextual Prx function is defined by its substrate specificity, reaction with enzymatic or nonenzymatic reductants, subcellular localization, and cell-, tissue-, and signal-specific expression. prx transcript regulation has been addressed in targeted approaches specifically aiming at exploring prx responses and in whole genome gene chip analyses aiming at understanding global transcriptome responses.

\section{A. Basal pattern of Prx expression and developmental control}

The cytosolic and mitochondrial type II Prxs are expressed in all plant tissues, including cell suspension cultures and cell calli. AtPrxII B mRNA is present in all plant tissues and expressed at high levels in reproductive tissues of buds, flowers, siliques, and seeds (20). At-PrxII C and At-PrxII D mRNAs are low in most tissues except flowers and buds. Plastid PrxII E mRNA is found in most tissues being particularly high in seedlings, buds and cells from suspension cultures and then with decreasing amount in siliques, stems, seeds, flowers, leaves, and roots (20). Using reporter gene constructs consisting of the PrxII promoters and $\beta$-glucuronidase, AtPrxII $C$ promoter activity was seen to be strongest in buds and mature pollen, that of PrxII D in mature and germinating pollen as well as fertilized ovules. The promoter of AtPrxII E mediates reporter expression in the stamen of young flowers, the embryo sac, and the albumen of seeds from maturing siliques (20).
Transcriptome analyses offer quite complete data sets for systems biology. Mathematical and statistical tools can be applied to these large data sets and reveal conditional regulatory dependencies and possibly interlinked biological functions (95). Coexpression analysis reveals that At-PrxIIC and PrxIID, which are not distinguished on the ATH1 gene chip, belong to a large group of highly coregulated transcripts, containing 259 transcripts sharing the very high Pearson correlation coefficient of $>0.9$ (100). Many coregulated transcripts are related to signaling such as mitogen activated protein kinases, Ca-binding proteins, GTPases, and transcription factors, to cell wall- and lipid-related genes and cytoskeleton (tubulin, actin). At-1-CysPrx transcript negatively correlates with many photosynthesis genes and as expected positively with transcripts coding for late embryogenesis abundant and seed storage proteins, dehydrins, and cell wall proteins. The group of highly coregulated transcripts $>0.9$ comprises 15 genes, with another set of 18 genes $>0.8$. Ma et al. (96) employed graphical Gaussian models (GGM) to search for partial coregulation networks from the Arabidopsis NASC database with about 22,200 genes, using more than 2000 gene chip experiments. The average network connectivity for a node (gene) was 5.5 (96). Figure 16 presents the result for five Prx genes. A coexpression network of 24 elements emerged with At-2-CysPrxA as seed and connected At2-CysPrxA expression to photosynthesis-related transcripts such as those for photosystem I reaction center subunit PSI-H, FeS subunit of cytochrome $b_{6} f$ complex, $\gamma$-subunit of ATP synthase, fructose-bisphosphate aldolase, glyceraldehyde 3-phosphate dehydrogenase, phosphate/triose-phosphate translocator, and also to ribosomal proteins, PrxII E and Cyp20-3. This result underpins the clear functional link between 2-CysPrxA and photosynthesis also at the level of gene regulation. Surprisingly, the network emerging with 2$C y \operatorname{Pr} x B$ only contains nodes to six other transcripts, including Apx4 and an inner envelope protein (Fig. 16B). The distinct regulation of both isogenes may hint to different functional context despite the fact that biochemical differences between At2-CysPrxA and B appear to be missing. The network centered around $\operatorname{Prx} Q$ transcript has 23 components and contains transcripts involved in light and dark reactions of photosynthesis, for example, photosynthetic pigment binding proteins (CP47, LHCB4.3, and SEP1), triose phosphate isomerase, but also protein synthesis and turnover with caseinolytic protease and nine ribosomal subunits and an elongation factor (Fig. 16C). The relationship between expression of ribosome subunits and Prxs is interesting since Sideri et al. (163) observed an association of yeast Prx with ribosomes and proposed a specific role for Prx in protecting ribosomal activity under oxidative stress. The network with At-PrxII E only contained At-2-CysPrxA, Cyp20-3, and a putative dicarboxylate transporter, whereas At-PrxII F associated with NADH-ubiquinone oxidoreductase (At5g52840) and two other transcripts (Fig. 16D, E; see also Supplementary Table S1; Supplementary Data are available online at www.liebertonline.com/ars).

Prx gene expression is under environmental and developmental control. The At-1-CysPrx expression occurs predominantly in aleurone layer and the embryo and also in specific vegetative tissues and is upregulated in the presence of ABA. Rice 1-CysPrx (R1C-Prx and OS1-CysPrx) is strongly expressed in imbibed rice seeds (91). Transcript amounts continuously decline from days 0 to 20 after imbibition when the 

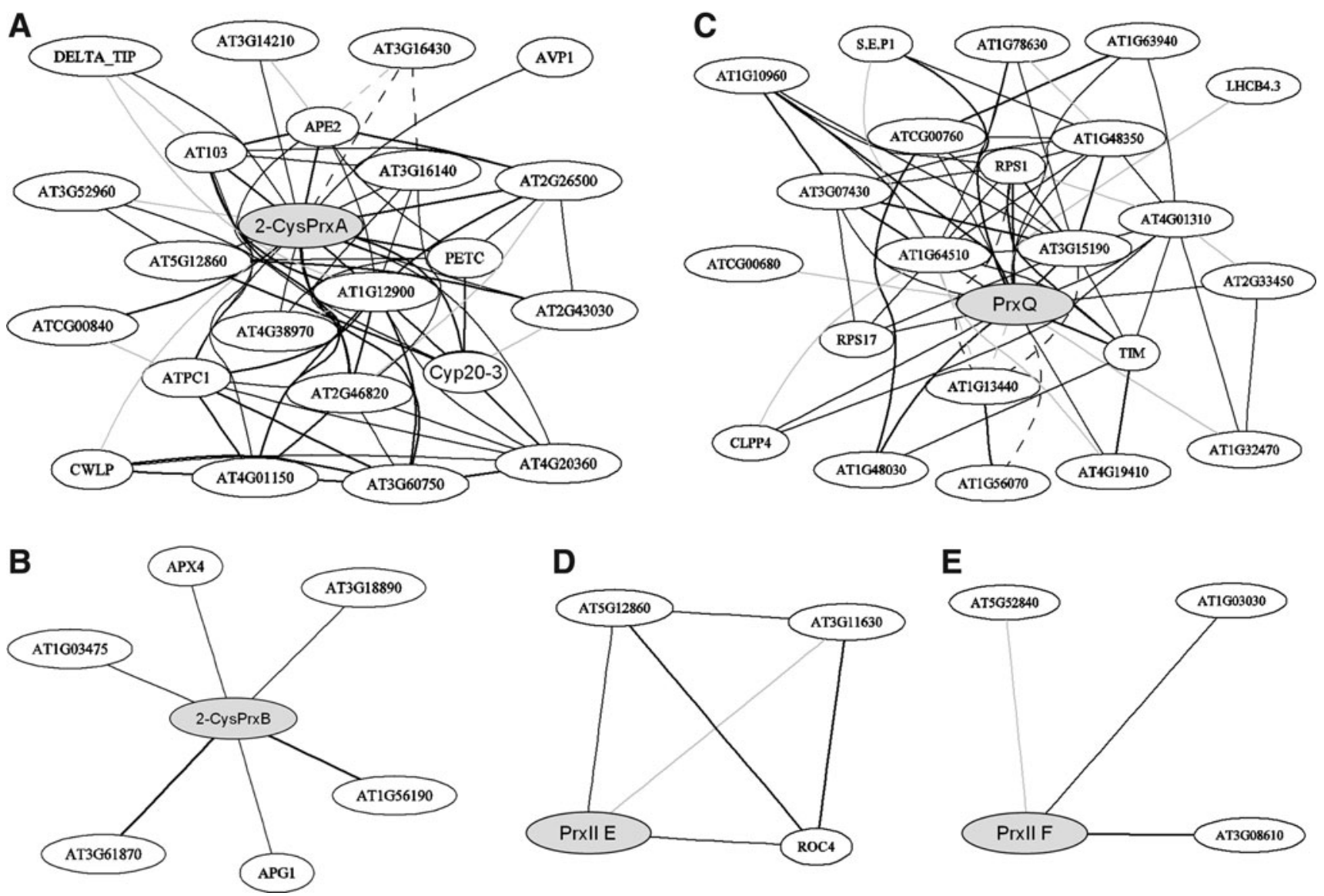

FIG. 16. Coregulation networks based on graphical Gaussian model (GGM) (96) as constructed through search with (A) At2-CysPrxA, (B) At2-CysPrxB, (C) AtPrxQ, (D) AtPrxII E, (E) AtPrxII F. As outlined by Ma et al. (96) cutoffs for partial correlation (pcor models) was set at less than -0.1 and larger than 0.1 corresponding to $p$-values lower than $1.92 \cdot 10^{-190}$. The list of network components is provided in Supplementary Table S1.

mRNA essentially is undetectable. Likewise, Os-1CysPrx protein amount is maximal at days $0-5$ and then declines as well to day 20. Haslekas et al. (53) analyzed the promoter of At-1-CysPrx for cis-regulatory elements and identified an antioxidant response promoter element (ARE) at position -363 $\mathrm{bp}$, which mediates an about fivefold stimulation of reporter activity upon treatment with dihydroxybenzene hydroquinone (HQ). In addition, an ABA-responsive element was detected that may be activated by cooperative interaction of ABA insensitive 3 (ABI3) and ABI5 (53). Consistent with the hypothesis that ABI3 controls At1-CysPrx expression, and ectopic expression of ABI3 enables At-1-CysPrx expression in vegetative tissues. In the resurrection plant Xerophyta viscosa, the $X v-1-C y s \operatorname{Pr} x$ has lost its predominant developmentally controlled expression pattern and is expressed throughout the vegetative tissue in a strongly stress-dependent manner (116).

\section{B. Regulation of plastid Prx expression by retrograde signaling}

Only few genes for plastid and mitochondrial proteins are encoded by the plastid and mitochondrial genomes. Therefore, coordination of nuclear gene expression with metabolic and developmental demand of the organelles is under control of signal exchange. Information and material flow from the nucleus/cytosol to the organelles is termed antherograde, the reverse direction from the organelles to the nucleus retrograde signaling $(126,134,139)$. All prx genes are encoded by the nuclear genome of higher plants. While transcript regulation of Prxs in response to stresses and effectors has been studied in quite some detail, involved signaling cascades have only been addressed for 1-CysPrx and 2-CysPrx. Regulation of 1CysPrx, which involves ABA-responsive elements and ABI3 and ABI5, has been described above in the paragraph on developmental control of gene expression. As to the latter example, 2-CysPrx has served as model to study redoxdependent retrograde signaling from the chloroplast to the nucleus $(10,56,60,161)$. The promoter of $A t-2-C y s P r x A$ drives $\beta$-glucuronidase expression in transgenic $A$. thaliana in dependence on the electron pressure downstream of photosystem I. This conclusion is based on the observation that at constant light intensity, the reporter activity is stimulated at low $\mathrm{CO}_{2}$ concentration and suppressed at $\mathrm{CO}_{2}$ concentration of $2000 \mu \mathrm{l} / \mathrm{l}$. High $\mathrm{CO}_{2}$ saturates the carboxylation reaction, maximally drains electrons by photochemistry and thus establishes less reducing conditions in the photosynthetic electron transport chain and stroma (10). Alternatively, increasing the light intensity at constant $\mathrm{CO}_{2}$ availability stimulates reporter activity. Consumption of electrons by nitrate reduction after $\mathrm{KNO}_{3}$ feeding also lowers At-2-CysPrx promoter activ- 
ity. The redox-responsive promoter region could be narrowed down to a 216-bp fragment, which also mediates the ABA, ascorbate, and wounding responses (10). As first established in the liverwort, Riccia fluitans protein kinases are implicated in signaling the metabolic redox cue to 2-CysPrx gene expression. Thus, incubation of submersed Riccia fluitans thali or A. thaliana leaf sections with the protein kinase inhibitor staurosporin partially suppresses the ascorbate-dependent downregulation of 2-CysPrx promoter activity $(10,60)$. Two subsequent studies employed the redox sensitive promoter of At-2-CysPrxA (p2CPA) to advance the understanding on the mechanism of redox control of At-2-CysPrx expression. LUC reporter gene was expressed in transgenic $A$. thaliana under control of the 2184-bp promoter fragment of p2CPA (56). The lines were chemically mutagenized and screened for mutants with constitutively underexpressed 2-CysPrx. Obtained independent mutant lines display disturbed redox balance (rimb mutants: redox imbalance), increased oxidation state of ascorbate, higher reduction of plastoquinone pool, increased oxidation of 2-CysPrx, and alterations in other redox-related traits. The identification of the mutations triggering the constitutive downregulation of 2-CysPrx expression despite increased oxidative stress are expected to shed light on signaling pathways that control 2-CysPrx expression (56). In a yeast-1-hybrid screen the transcription factor RAP2.4a was identified and confirmed as binding partner and regulator of the redox box of the At-2-CysPrxA promoter (161). AtRAP2.4a transactivates the p2CPA promoter in a mesophyll protoplast-based transient expression system. A T-DNA insertion line with disrupted rap2.4a gene reveals disturbed expression of a set of antioxidant genes and a growth phenotype different from wild type under fluctuating growth conditions (161). AtRAP2.4a belongs to the large family of 147 APETALA 2/ethylene response element binding protein transcription factors in A. thaliana, which are known to be involved in regulation of stress responses and retrograde signaling (43). The role and regulation of AtRAP2.4a or related APETALA 2/ethylene response element binding protein transcription factors in retrograde and stress-dependent signaling to At2-CysPrx will need further attention and may pave the way to the first mechanistic concept of prx gene expression in plants.

\section{Stress-dependent regulation}

Abiotic and biotic stresses usually interfere with the redox state of the cells and often elicit generation of excess ROS and RNS (4, 71, 107, 174). Prx transcript regulation has been studied in quite some detail and shows regulatory variation in dependence on the type of Prx, plant species, stress-intensity, and developmental state. Prx transcripts respond to all kind of stresses, including low and high light, salinity, heavy metals, nutrient deprivation, temperature extremes, and chemical effectors. It is beyond the scope of this review to cover all these findings; thus, only a few examples are given. Regulation of PrxQ amounts involves strong transcript level adjustment; for example, PrxQ1 and PrxQ2 transcript amounts in Synechocystis PPC6803 increase in response to $\mathrm{H}_{2} \mathrm{O}_{2}$, high light, or nutrient deprivation $(132,170)$. Likewise, AtPrxQ transcript levels are upregulated during oxidative stress (62). In general, $\operatorname{Prx} Q$ transcript appears to be the most responsive one among the four plastid prx genes in A. thaliana. PrxQ transcript ac- cumulates upon peroxide stress and decreases under reducing conditions $(61,62)$. In line with this transcriptional regulation, PrxQ protein amounts increase 1.8-2.8-fold in thylakoids isolated from effector-infiltrated leaves treated with $\mathrm{H}_{2} \mathrm{O}_{2}$, diamide or t-BOOH (137). Thylakoid PrxQ also increases in leaves infiltrated with ascorbate or at low light, but decreased in high light (137).

Poplar Prx-Q transcript and protein amounts respond to the fungal pathogen Melampsora laricii-populina, the causative agent of the poplar rust (149). They increase upon infection with a pathogenic race eliciting hypersensitive response but decrease during a compatible interaction between poplar and the rust fungus $M$. laricii-populina (149).

The 1-CysPrx transcript of the dehydration-tolerant Xerophyta viscosa is absent in fully hydrated tissue. Transcript accumulates in tissues under abiotic stresses such as dehydration, heat $\left(42^{\circ} \mathrm{C}\right)$, high light intensity $(1500 \mu \mathrm{mol}$ quanta $\left.\mathrm{m}^{-2} \mathrm{~s}^{-1}\right), \mathrm{NaCl}(100 \mathrm{mM})$, and when treated with ABA $(100 \mu M)$. Changes in $\mathrm{X}_{v}-1-C y s P r x$ protein levels correlate well with transcript regulation (116). This result indicates a significant role of 1-CysPrx in extreme drought tolerance such as in resurrection plants and also shows that unusual regulation of Prx expression might contribute to special stress tolerance features.

In A. thaliana water deficit, chilling, and senescence only lead to small increases in PrxII F protein abundance, whereas photo-oxidative conditions or heavy metal treatment were uneffective (49). This result contrasted that by Finkemeier et al. (46), who observed increased AtPrxII F protein and transcript amounts in roots of Cd-treated plants. In pea leaves PrxII F protein accumulates upon cold and heavy-metal treatment (13). All these observations suggest that PrxII F functions in the general antioxidant defense of the plant mitochondrion with significance for ROS- and RNS-dependent signaling. Low light intensities decrease transcript amounts of the plastidic At-2-CysPrxA, At-2-CysPrxB, At-PrxQ, and cytosolic At-PrxII B, whereas plastidic At-PrxII $E$ and mitochondrial At-PrxII $F$ remain unaltered (62). The transcriptional response of the leaf antioxidant defense system was studied in nutrient-deprived, hydroponically grown A. thaliana (75). At-PrxII E, F and At-PrxQ transcripts are unresponsive to P-, $\mathrm{N}-$, and S-deficiency. Strong upregulation of At-PrxIIC and $D$ occurs under insufficient $\mathrm{P}$ nutrition both in young and old leaves.

This incomplete list of stresses that affect Prx gene expression demonstrates interesting correlations between various environmental perturbances and Prx gene expression and, in some studies, protein amounts. Manipulation of Prx amounts in transgenic plants offers access to a more causal understanding of these dependencies. Thus, the severe root growth inhibition of PrxII F-deficient $A$. thaliana lines in the presence of $\mathrm{Cd}$ and of salicylhydroxamic acid, an inhibitor of cyanideinsensitive respiration, indicates the importance of PrxII Fmediated ROS detoxification under conditions of abiotic stress (46). In another set of experiments, plants were produced that overexpress selected Prxs. Transgenic grass plants of Festuca arundinacea that overexpress 2-CysPrx are more tolerant to heat stress of $42^{\circ} \mathrm{C}$ and methylviologen treatment, respectively, as determined by decreased ion leakage, indicating less membrane damage, less inhibition of ФPSII and less lipid peroxidation (78). The authors conclude that elevated levels of 2-CysPrx protect the plant tissue under stress 
either by increased peroxide detoxification capacity or by improved chaperone activity. At present it remains to be clarified which of both mechanisms stimulates stress tolerance of the transgenes. Kiba et al. (77) cloned PrxQ from Gentiana triflora GtPrxQ, originally identified as antifungal protein GfAFP1, and produced transgenic tobacco significantly overexpressing GtPrxQ. Leaves from the transgenic lines better resist to methylviologen treatment and show improved tolerance to infection with the fungal pathogen Botrytis cinerea (77). In another study, overexpression of PrxQ from Suaeda salsa, SsPrxQ, in A. thaliana supported performance of the transgenic lines during saline and cold stress (72). The results from transgenic approaches strengthen the notion that Prx function is linked to stress tolerance. However, in all these studies, the characterization remained on a preliminary level and neither addressed the tradeoffs of the plants for overexpressing 2-CysPrx or PrxQ nor provided any detailed analyses on responses in dependence of stress duration and intensities, nor explored the mechanisms involved in response elicitation.

\section{Additional Functions of Peroxiredoxins in Plants}

Most studies on Prx function focused on leaves and sometimes roots. Only few investigations addressed other developmental stages such as seedlings, nodules, reproductive organs, and fruits. This section describes reports on Prx expression in $A$. thaliana seedlings, pea nodules, and pea fruits. Upon seed germination and during early seedling development, Prxs are rapidly activated at the transcription level and accumulate to high protein amounts before other antioxidant systems such as ascorbate peroxidase (131). In fact one of the first 2-CysPrx BAS1 (initial name for plant 2CysPrx) was identified from barley in a screen for genes highly expressed in the basal growing zone of gras leaves (6). In accordance with this assumed role in early development of seedlings, $A$. thaliana antisense lines expressing the 2-CysPrx gene in antisense orientation had a peculiar phenotype in early seedling establishment and development when grown on plates (8). Legumes develop a symbiosis with $\mathrm{N}_{2}$-fixing rhizobacteria. Western blot analyses reveal the presence of mitochondrial PrxIIF in pea nodules (51). The abundance of PrxIIF is unaffected by nodule aging or by application of external ascorbic acid. PrxQ and 2-CysPrx are absent from the nodules. An antibody directed against the cytosolic AtPrxIIC of $17 \mathrm{kDa}$ failed to recognize the homologous protein but labeled a $68 \mathrm{kDa}$ band. In 9-week-old nodules this band increases upon external feeding of ascorbate. The data show that Prx are present in nodules and might respond to the antioxidant state of the nodule cells. Nodule mitochondria sustain the energy-intensive process of nitrogen fixation, but at the same time release ROS at high rates. PrxIIF appears to play a role in this antioxidant defense network of nodules in addition to ascorbate peroxidase, heme peroxidase, and catalase (69). Fruits of snap pea (Pisum sativum) grown under nodulating and non-nodulating conditions were classified in three maturity stages. Prxs were quantified in the seeds and seedless pods by Western blot analysis (103). Ps2-CysPrx protein is detected in pods, whereas PsPrxQ is absent. PsPrxII F and cytosolic PrxII are present in seeds and pods. Cytosolic PrxIIs decrease in seeds, whereas they increase in pods during storage from immature to mature and over- mature pea fuits. The authors suggest that maturation and storage of pea fruits entails a simultaneous downregulation of ROS as well as antioxidant levels, thereby avoiding the development of oxidative stress. Cytosolic PrxII are proposed to play a significant role in this process of fruit development and ripenening (103).

\section{Outlook}

Fifteen years of plant Prx research has clarified the enzymatic activity as peroxidase, the interactor activity as chaperone and binding partner, and the redox-dependent conformational dynamics. In terms of understanding the peroxidase activity, the maximal catalytic efficiencies of plant Prx need to be determined by stop flow or other time-resolved methods. In addition, substantial evidence has been put forward to assign additional roles in cell signaling to Prx also in plants. Significant coexpression, proof of interactions, activity tests, and evolutionary conservation have proven that Prxs and their interacting partners form machines or modules in defense and signaling. The best studied example is the 2CysPrx, which forms a module together with Trx or Trx-like protein, sulfiredoxin, and cyclophilin. The role of the chloroplast Cyp20-3 in this sensory module is not resolved but it is proposed that Cyp20-3 could play a role in disassembly of hyperaggregated 2-CysPrx complexes, for example, to ease regeneration (118). Likewise, the cellular function of Srx as regulatory interactor should be investigated since it controls 2-CysPrx activity by retroreduction (73). The immediate effect of 2-CysPrx on photosystem II is not understood. 2-CysPrx could be involved in localized peroxide detoxification or affect properties of the photosystem such as stability or turnover, and also the significance of the chaperone activity should be addressed under regular and stress conditions. In the end, the Prx-module within the redox regulatory network will have to be described by mathematical models, allowing us to simulate and predict its state and function. The simulation may provide explanations why, for example, distinct Grx and Trx branches exist in the thiol-disulfide redox network each with associated Prxs (Fig. 7) and how they equilibrate by multiple reactions with distinct kinetic constraints.

To understand the regulation of Prx functions in plant defense and signaling, the posttranslational modifications of plant and cyanobacterial Prxs need to be investigated in more detail by proteome analysis. Reversible phosphorylation, acetylation, nitration, and glutathionylation of Prxs and also of their binding partners represent important mechanisms that may control the activity and functionality of the signaling modules. It is known that genetic manipulation of Prx amounts and activities, for example, of 2-CysPrx, PrxQ, and PrxII F, induces changes in nuclear gene expression. These changes appear to be specific for each Prx and different from ROSinduced signaling. The pathways of signal transmission from the Prx modules to the target genes need to be resolved by target-up and signal-down dissection of the involved elements. The roles of Prxs in cell signaling should be differentiated between protein-specific initiation of signaling and effects on ROS and RNS signaling. In turn, regulation of Prx expression on the promoter and signaling level has only been investigated for At1-CysPrx and At2-CysPrx. Cis-elements and transcription factors are of interest that couple expressional regulation of the other Prxs to metabolic and environmental cues. 
The least investigated $\operatorname{Prx}(\mathrm{s})$ are the cytosolic PrxII, which are present as three isoforms in A. thaliana, two isoforms in poplar, and with only one representative in rice (Table 1 ). The high similarity of the gene sequences might allow for RNAi or miRNA approaches in $A$. thaliana to suppress all isoforms simultaneously. Alternatively, the significance of PrxII B-like cytosolic PrxII could be addressed in rice. Such approaches will address the functions of Prxs in metabolism and development of organs other than leaves and roots, which have been studied in most detail up to now.

\section{Acknowledgments}

This work was supported by the Deutsche Forschungsgemeinschaft and Sonderforschungsbereich 613 (A5, D9). Contributions and discussion by Dr. Janine König and Dr. Andrea Viehhauser are gratefully acknowledged.

\section{References}

1. Aalen RB, Opsahl-Ferstad HG, Linnestad C, and Olsen OA. Transcripts encoding an oleosin and a dormancy-related protein are present in both the aleurone layer and the embryo of developing barley (Hordeum vulgare L.) seeds. Plant J 5: 385-396, 1994.

2. Akerman SE and Müller S. Peroxiredoxin-linked detoxification of hydroperoxides in Toxoplasma gondii. J Biol Chem 280: 564-570, 2005.

3. Aran M, Caporaletti D, Senn AM, Tellez de Iñon MT, Girotti MR, Llera AS, and Wolosiuk RA. ATP-dependent modulation and autophosphorylation of rapeseed 2-Cys peroxiredoxin. FEBS J 275: 1450-1463, 2008.

4. Arasimowicz M and Floryszak-Wieczorek J. Nitric oxide as a bioactive signalling molecule in plant stress responses. Plant Sci 172: 876-887, 2007.

5. Arsova B, Hoja U, Wimmelbacher M, Greiner E, Ustün S, Melzer M, Petersen K, Lein W, and Börnke F. Plastidial thioredoxin $\mathrm{z}$ interacts with two fructokinase-like proteins in a thiol-dependent manner: evidence for an essential role in chloroplast development in Arabidopsis and Nicotiana benthamiana. Plant Cell 22: 1498-1515, 2010.

6. Baier M and Dietz KJ. Primary structure and expression of plant homologues of animal and fungal thioredoxindependent peroxide reductases and bacterial alkyl hydroperoxide reductases. Plant Mol Biol 31: 553-564, 1996.

7. Baier M and Dietz KJ. The plant 2-Cys peroxiredoxin BAS1 is a nuclear-encoded chloroplast protein: its expressional regulation, phylogenetic origin, and implications for its specific physiological function in plants. Plant J 12: 179-190, 1997.

8. Baier M and Dietz KJ. Protective function of chloroplast 2cysteine peroxiredoxin in photosynthesis. Evidence from transgenic Arabidopsis. Plant Physiol 119: 1407-1414, 1999.

9. Baier M, Noctor G, Foyer $\mathrm{CH}$, and Dietz KJ. Antisense suppression of 2-cysteine peroxiredoxin in Arabidopsis specifically enhances the activities and expression of enzymes associated with ascorbate metabolism but not glutathione metabolism. Plant Physiol 124: 823-832, 2000.

10. Baier M, Ströher E, and Dietz KJ. The acceptor availability at photosystem I and ABA control nuclear expression of 2Cys peroxiredoxin-A in Arabidopsis thaliana. Plant Cell Physiol 45: 997-1006, 2004.

11. Banks JA. Selaginella and 400 million years of separation. Annu Rev Plant Biol 60: 223-238, 2009.
12. Barranco-Medina S, Kakorin S, Lazaro JJ, and Dietz KJ. Thermodynamics of the dimer-decamer transition of reduced human and plant 2-Cys peroxiredoxin. Biochemistry 47: 7196-7204, 2008.

13. Barranco-Medina S, Krell T, Bernier-Villamor L, Sevilla F, Lazaro JJ, and Dietz KJ. Hexameric oligomerization of mitochondrial peroxiredoxin PrxIIF and formation of an ultrahigh affinity complex with its electron donor thioredoxin Trx-o. J Exp Bot 59: 3259-3269, 2008.

14. Barranco-Medina S, Lazaro JJ, and Dietz KJ. The oligomeric conformation of peroxiredoxins links redox state to function. FEBS Lett 583: 1809-1816, 2009.

15. Barranco-Medina S, Lopez-Jaramillo FJ, Bernier-Villamor L, Sevilla F, and Lazaro JJ. Cloning, overexpression, purification, and preliminary crystallographic studies of a mitochondrial type II peroxiredoxin from Pisum sativum. Acta Crystallogr F62, 695-698, 2006.

16. Berberich T, Uebler $M$, and Feierabend J. Cloning of a cDNA encoding a thioredoxin peroxidase (TPx) homolog from winter rye (Secale cereale L.) (Accession No. AF076920) (PGR98-167). Plant Physiol 118: 711, 1998.

17. Bernier-Villamor L, Navarro E, Sevilla F, and Lázaro JJ. Cloning and characterization of a 2-Cys peroxiredoxin from Pisum sativum. J Exp Bot 55: 2191-2199, 2004.

18. Bernroitner M, Zamocky M, Furtmüller PG, Peschek GA, and Obinger $\mathrm{C}$. Occurrence, phylogeny, structure, and function of catalases and peroxidases in cyanobacteria. $J$ Exp Bot 60: 423-440, 2009.

19. Bonfoco E, Krainc D, Ankarcrona M, Nicotera P, and Lipton SA. Apoptosis and necrosis: two distinct events induced, respectively, by mild and intense insults with $\mathrm{N}$-methyl-D-aspartate or nitric oxide/superoxide in cortical cell cultures. Proc Natl Acad Sci USA 92: 7162-7166, 1995.

20. Bréhélin C, Meyer EH, de Souris JP, Bonnard G, and Meyer Y. Resemblance and dissemblance of Arabidopsis type II peroxiredoxins: similar sequences for divergent gene expression, protein localization, and activity. Plant Physiol 132: 2045-2057, 2003.

21. Broin M, Cuiné S, Eymery F, and Rey P. The plastidic 2Cys-peroxiredoxin is a target for a thioredoxin involved in the protection of the photosynthetic apparatus against oxidative damage. Plant Cell 14: 1417-1432, 2002.

22. Broin M, Cuiné S, Peltier G, and Rey P. Involvement of CDSP 32, a drought-induced thioredoxin, in the response to oxidative stress in potato plants. FEBS Lett 467: 245-248, 2000.

23. Broin $M$ and Rey P. Potato plants lacking the CDSP32 plastidic thioredoxin exhibit over-oxidation of the BAS1 2cys peroxiredoxin and increased lipid peroxidation in thylakoids under photooxidative stress. Plant Physiol 132: 1335-1343, 2003.

24. Bryk R, Griffin P, and Nathan C. Peroxynitrite reductase activity of bacterial peroxiredoxins. Nature 407: 211-215, 2000.

25. Caporaletti D, D'Alessio AC, Rodriguez-Suarez RJ, Senn AM, Duek PD, Wolosiuk RA. Non-reductive modulation of chloroplast fructose-1,6-bisphosphatase by 2-Cys peroxiredoxin. Biochem Biophys Res Commun 355: 722-727, 2007.

26. Chae HZ, Kim IH, and Rhee SG. Cloning, sequencing, and mutation of thiol-specific antioxidant gene Saccharomyces cerevisiae. J Biol Chem 268: 16815-16821, 1993.

27. Chang TS, Jeong W, Choi SY, Yu S, Kang SW, and Rhee SG. Regulation of peroxiredoxin I activity by Cdc2-mediated phosphorylation. J Biol Chem 277: 25370-25376, 2002. 
28. Chen JW, Dodia C, Feinstein SI, Jain MK, and Fisher AB. 1Cys peroxiredoxin, a bifunctional enzyme with glutathione peroxidase and phospholipase A2 activities. J Biol Chem 275: 28421-28427, 2000.

29. Chenna R, Sugawara H, Koike T, Lopez R, Gibson TJ, Higgins DG, and Thompson JD. Multiple sequence alignment with the Clustal series of programs. Nucleic Acids Res 31: 3497-3500, 2003.

30. Chiadmi M, Navaza A, Miginiac-Maslow M, Jacquot JP, and Cherfils J. Redox signalling in the chloroplast: structure of oxidized pea fructose-1,6-bisphosphate phosphatase. EMBO J 18: 6809-6815, 1999.

31. Choi YO, Cheong NE, Lee KO, Jung BG, Hong CH, Jeong JH, Chi YH, Kim K, Cho MJ, and Lee SY. Cloning and expression of a new isotype of the peroxiredoxin gene of Chinese cabbage and its comparison to 2Cys-peroxiredoxin isolated from the same plant. Biochem Biophys Res Commun 258: 768-771, 1999.

32. Claus J, Knight I, and Winyard PG. Aspects of the biological redox chemistry of cysteine: from simple redox responses to sophisticated signalling pathways. Biol Chem 387: 1385-1397, 2006.

33. Cline MS, Smoot M, Cerami E, Kuchinsky A, Landys N, Workman C, Christmas R, Avila-Campilo I, Creech M, Gross B, et al. Integration of biological networks and gene expression data using Cytoscape. Nat Protoc 2: 2366-2382, 2007.

34. Cock JM, Sterck L, Rouzé P, Scornet D, Allen AE, Amoutzias G, Anthouard V, Artiguenave F, Aury JM, Badger JH, et al. The ectocarpus genome and the independent evolution of multicellularity in brown algae. Nature 465: 617-621, 2010.

35. Collin V, Issakidis-Bourguet E, Marchand C, Hirasawa M, Lancelin JM, Knaff DB, and Miginiac-Maslow M. The Arabidopsis plastidial thioredoxins: new functions and new insights into specificity. J Biol Chem 278: 23747-23752, 2003.

36. Collin V, Lamkemeyer P, Miginiac-Maslow M, Hirasawa M, Knaff DB, Dietz KJ, and Issakidis-Bourguet E. Characterization of plastidial thioredoxins from Arabidopsis belonging to the new y-type. Plant Physiol 136: 4088-4095, 2004.

37. Delledonne M, Zeier J, Marocco A, and Lamb C. Signal interactions between nitric oxide and reactive oxygen intermediates in the plant hypersensitive disease resistance response. Proc Natl Acad Sci USA 98: 13454-13459, 2001.

38. Demirkaya M, Dietz KJ, and Sivritepe HO. Changes in antioxidant enzymes during ageing of onion seeds. Notulae Botanicae Horti Agrobotanici Clui-Napoca 38: 49-52, 2010.

39. Dietz KJ. Redox signal integration: from stimulus to networks and genes. Physiol Plant 133: 459-468, 2008.

40. Dietz KJ. The dual function of plant peroxiredoxins in antioxidant defence and redox signalling. Subcell Biochem 44: 267-294, 2007.

41. Dietz KJ, Jacquot JP, and Harris G. Hubs and bottlenecks in plant molecular signalling networks. New Phytol 188: 919938, 2010.

42. Dietz KJ, Jacob S, Oelze ML, Laxa M, Tognetti V, de Miranda SM, Baier M, and Finkemeier I. The function of peroxiredoxins in plant organelle redox metabolism. J Exp Bot 57: 1697-1709, 2006.

43. Dietz KJ, Vogel MO, and Viehhauser A. AP2/EREBP transcription factors are part of gene regulatory networks and integrate metabolic, hormonal and environmental signals in stress acclimation and retrograde signalling. Protoplasma 245: 3-14, 2010.

44. Dixon DP, Skipsey M, Grundy NM, and Edwards R. Stressinduced protein S-glutathionylation in Arabidopsis. Plant Physiol 138: 2233-2244, 2005.

45. Echalier A, Trivelli X, Corbier C, Rouhier N, Walker O, Tsan P, Jacquot JP, Aubry A, Krimm I, and Lancelin JM. Crystal structure and solution NMR dynamics of a D (type II) peroxiredoxin glutaredoxin and thioredoxin dependent: a new insight into the peroxiredoxin oligomerism. Biochemistry 44: 1755-1767, 2005.

46. Finkemeier I, Goodman M, Lamkemeyer P, Kandlbinder A, Sweetlove LJ, and Dietz KJ. The mitochondrial type II peroxiredoxin $\mathrm{F}$ is essential for redox homeostasis and root growth of Arabidopsis thaliana under stress. J Biol Chem 280: 12168-12180, 2005.

47. Foyer $\mathrm{CH}$ and Noctor G. Redox regulation in photosynthetic organisms: signalling, acclimation, and practical implications. Antioxid Redox Signal 11: 861-905, 2009.

48. Gama F, Bréhélin C, Gelhaye E, Meyer Y, Jacquot JP, Rey P, and Rouhier N. Functional analysis and expression characteristics of chloroplastic Prx IIE. Physiol Plant 133: 599610, 2008.

49. Gama F, Keech O, Eymery F, Finkemeier I, Gelhaye E, Per Gardeström P, Dietz KJ, Rey P, Jacquot JP, and Rouhier N. The mitochondrial type II peroxiredoxin from poplar. Physiol Plant 129: 196-206, 2007.

50. Goldmark PJ, Curry J, Morris CF, and Walker-Simmons MK. Cloning and expression of an embryo-specific mRNA up-regulated in hydrated dormant seeds. Plant Mol Biol 19: 433-441, 1992.

51. Groten K, Dutilleul C, van Heerden PDR, Vanacker N, Bernard S, Finkemeier I, Dietz KJ, and Foyer CH. Redox regulation of peroxiredoxin and proteinases by ascorbate and thiols during pea root nodule senescence. FEBS Lett 580: 1269-1276, 2006.

52. Hall A, Karplus PA, and Poole LB. Typical 2-Cys peroxiredoxins - structures, mechanisms and functions. FEBS J 276: 2469-2477, 2009.

53. Haslekås C, Grini PE, Nordgard SH, Thorstensen T, Viken MK, Nygaard V, and Aalen RB. ABI3 mediates expression of the peroxiredoxin antioxidant AtPER1 gene and induction by oxidative stress. Plant Mol Biol 53: 313-326, 2003.

54. Haslekås C, Stacy RA, Nygaard V, Culiáñez-Macià FA, and Aalen RB. The expression of a peroxiredoxin antioxidant gene, AtPer1, in Arabidopsis thaliana is seed-specific and related to dormancy. Plant Mol Biol 36: 833-845, 1998.

55. Haslekås C, Viken MK, Grini PE, Nygaard V, Nordgard $\mathrm{SH}$, Meza TJ, and Aalen RB. Seed 1-cysteine peroxiredoxin antioxidants are not involved in dormancy, but contribute to inhibition of germination during stress. Plant Physiol 133: 1148-1157, 2003.

56. Heiber I, Ströher E, Raatz B, Busse I, Kahmann U, Bevan MW, Dietz KJ, and Baier M. The redox imbalanced mutants of Arabidopsis differentiate signalling pathways for redox regulation of chloroplast antioxidant enzymes. Plant Physiol 143: 1774-1788, 2007.

57. Hofmann B, Hecht HJ, and Flohé L. Peroxiredoxins. Biol Chem 383: 347-364, 2002.

58. Holmgren A. Thioredoxin. Annu Rev Biochem 54: 237-271, 1985.

59. Holmgren A. Thioredoxin catalyzes the reduction of insulin disulfides by dithiothreitol and dihydrolipoamide. J Biol Chem 254: 9627-9632, 1979. 
60. Horling F, Baier M, and Dietz KJ. Redox-regulation of the expression of the peroxide-detoxifying chloroplast 2-cys peroxiredoxin in the liverwort Riccia fluitans. Planta 214: 304-313, 2001.

61. Horling F, König J, and Dietz KJ. Type II peroxiredoxin C, a member of the peroxiredoxin family of Arabidopsis thaliana: its expression and activity in comparison with other peroxiredoxins. Plant Physiol Biochem 40: 491-499, 2002.

62. Horling F, Lamkemeyer P, König J, Finkemeier I, Kandlbinder A, Baier M, and Dietz KJ. Divergent light-, ascorbate-, and oxidative stress-dependent regulation of expression of the peroxiredoxin gene family in Arabidopsis. Plant Physiol 131: 317-325, 2003.

63. Horta BB, de Oliveira MA, Discola KF, Cussiol JR, and Netto LE. Structural and biochemical characterization of peroxiredoxin Qbeta from Xylella fastidiosa: catalytic mechanism and high reactivity. J Biol Chem 285: 1605116065, 2010.

64. Hosoya-Matsuda N, Motohashi K, Yoshimura H, Nozaki A, Inoue K, Ohmori M, and Hisabori T. Anti-oxidative stress system in cyanobacteria. Significance of type II peroxiredoxin and the role of 1-Cys peroxiredoxin in Synechocystis sp. strain PCC6803. J Biol Chem 280, 840-846, 2005.

65. Hugo M, Turell L, Manta B, Botti H, Monteiro G, Netto LE, Alvarez B, Radi R, and Trujillo $\mathrm{M}$. Thiol and sulfenic acid oxidation of $\mathrm{AhpE}$, the one-cysteine peroxiredoxin from Mycobacterium tuberculosis: kinetics, acidity constants, and conformational dynamics. Biochem 48: 9416-9426, 2009.

66. Iglesias-Baena I. Caracterizacion bioquimica y molecular del sistema antioxidante peroxirredoxina-sulfirredoxina. Doctoral thesis, Granada, Spain: Universidad de Granada, 2010.

67. Iglesias-Baena I, Barranco-Medina S, Lázaro-Payo A, López-Jaramillo FJ, Sevilla F, and Lázaro JJ. Characterization of plant sulfiredoxin and role of sulphinic form of 2-Cys peroxiredoxin. J Exp Bot 61: 1509-1521, 2010.

67a. Iglesias-Baena I, Barranco-Medina S, Sevilla F, Lázar JJ. The dual targeted plant Sulfiredoxin retroreduces the sulfinic form of atypical mitochondrial peroxiredoxin. Plant Physiol 155: 944-955, 2011.

68. Ischiropoulos $\mathrm{H}$ and al-Mehdi AB. Peroxynitrite-mediated oxidative protein modifications. FEBS Lett 364: 279-282, 1995.

69. Iturbe-Ormaetxe I, Matamoros MA, Rubio MC, Dalton DA, and Becana M. The antioxidants of legume nodule mitochondria. Mol Plant Microbe Interact 14: 1189-1196, 2001.

70. Jang HH, Lee KO, Chi YH, Jung BG, Park SK, Park JH, Lee JR, Lee SS, Moon JC, Yun JW, Choi YO, Kim WY, Kang JS, Cheong GW, Yun DJ, Rhee SG, Cho MJ, and Lee SY. Two enzymes in one; two yeast peroxiredoxins display oxidative stress-dependent switching from a peroxidase to a molecular chaperone function. Cell 117: 625-635, 2004.

71. Jaspers $P$ and Kangasjarvi J. Reactive oxygen species in abiotic stress signalling. Physiol Plant 138: 405-413, 2010.

72. Jing LW, Chen SH, Guo XL, Zhang $\mathrm{H}$, and Zhao $\mathrm{YX}$. Overexpression of a chloroplast-located peroxiredoxin $Q$ gene, SsPrxQ, increases the salt and low-temperature tolerance of Arabidopsis. J Integr Plant Biol 48: 1244-1249, 2006.

73. Jönsson TJ, Johnson LC, and Lowther WT. Structure of the sulphiredoxin-peroxiredoxin complex reveals an essential repair embrace. Nature 451: 98-101, 2008.

74. Jönsson TJ, Murray MS, Johnson LC, and Lowther WT. Reduction of cysteine sulfinic acid in peroxiredoxin by sulfiredoxin proceeds directly through a sulfinic phosphoryl ester intermediate. J Biol Chem 283: 23846-23851, 2008.

75. Kandlbinder A, Finkemeier I, Wormuth D, Hanitzsch M, and Dietz KJ. The antioxidant status of photosynthesizing leaves under nutrient deficiency: redox regulation, gene expression and antioxidant activity in Arabidopsis thaliana. Physiol Plant 120: 63-73, 2004.

76. Kaneko T, Sato S, Kotani H, Tanaka A, Asamizu E, Nakamura Y, Miyajima N, Hirosawa M, Sugiura M, Sasamoto S, Kimura T, Hosouchi T, Matsuno A, Muraki A, Nakazaki N, Naruo K, Okumura S, Shimpo S, Takeuchi C, Wada T, Watanabe A, Yamada M, Yasuda M, and Tabata S. Sequence analysis of the genome of the unicellular cyanobacterium Synechocystis sp. strain PCC6803. II. Sequence determination of the entire genome and assignment of potential protein-coding regions. DNA Res 3: 109-136, 1996.

77. Kiba A, Nishihara M, Tsukatani N, Nakatsuka T, Kato Y, and Yamamura S. A peroxiredoxin $\mathrm{Q}$ homolog from gentians is involved in both resistance against fungal disease and oxidative stress. Plant Cell Physiol 46: 1007-1015, 2005.

78. Kim KH, Alam I, Lee KW, Sharmin SA, Kwak SS, Lee SY, and Lee $\mathrm{BH}$. Enhanced tolerance of transgenic tall fescue plants overexpressing 2-Cys peroxiredoxin against methyl viologen and heat stresses. Biotechnol Lett 32: 571-576, 2010.

79. Kim SY, Jang HH, Lee JR, Sung NR, Bin Lee H, Lee DH, Park DJ, Kang CH, Chung WS, Lim CO, Yun DJ, Kim WY, Lee $\mathrm{KO}$, and Lee SY. Oligomerization and chaperone activity of a plant 2-Cys peroxiredoxin in response to oxidative stress. Plant Sci 177: 227-232, 2009.

80. Kirchsteiger K, Pulido P, González M, and Cejudo FJ. NADPH Thioredoxin reductase $C$ controls the redox status of chloroplast 2-Cys peroxiredoxins in Arabidopsis thaliana. Mol Plant 2: 298-307, 2009.

81. Klotz LO, Schröder P, and Sies H. Peroxynitrite signalling: receptor tyrosine kinases and activation of stress-responsive pathways. Free Radic Biol Med 33: 737-743, 2002.

82. Klughammer B, Baier M, and Dietz KJ. Inactivation by gene disruption of 2-cysteine-peroxiredoxin in Synechocystis sp. PCC 6803 leads to increased stress sensitivity. Physiol Plant 104: 699-706, 1998.

83. Kobayashi M, Ishizuka T, Katayama M, Kanehisa M, Bhattacharyya-Pakrasi M, Pakrasi HB, and Ikeuchi M. Response to oxidative stress involves a novel peroxiredoxin gene in the unicellular cyanobacterium Synechocystis sp. PCC 6803. Plant Cell Physiol 45: 290-299, 2004.

84. Koeck T, Fu X, Hazen SL, Crabb JW, Stuehr DJ, and Aulak KS. Rapid and selective oxygen-regulated protein tyrosine denitration and nitration in mitochondria. J Biol Chem 279: 27257-27262, 2004.

85. Kong W, Shiota S, Shi Y, Nakayama H, and Nakayama K. A novel peroxiredoxin of the plant Sedum lineare is a homologue of Escherichia coli bacterioferritin co-migratory protein (Bcp). Biochem J 351: 107-114, 2000.

86. König J, Baier M, Horling F, Kahmann U, Harris G, Schürmann P, and Dietz KJ. The plant-specific function of 2-Cys peroxiredoxin-mediated detoxification of peroxides in the redox-hierarchy of photosynthetic electron flux. Proc Natl Acad Sci USA 99: 5738-5743, 2002.

87. König J, Lotte K, Plessow R, Brockhinke A, Baier M, and Dietz KJ. Reaction mechanism of plant 2-Cys peroxiredoxin. Role of the $\mathrm{C}$ terminus and the quaternary structure. J Biol Chem 278: 24409-24420, 2003.

88. Lamkemeyer P, Laxa M, Collin V, Li W, Finkemeier I, Schöttler MA, Holtkamp V, Tognetti VB, Issakidis-Bourguet 
E, Kandlbinder A, Weis E, Miginiac-Maslow M, and Dietz KJ. Peroxiredoxin Q of Arabidopsis thaliana is attached to the thylakoids and functions in context of photosynthesis. Plant J 45: 968-981, 2006.

89. Laxa M, König J, Dietz KJ, and Kandlbinder A. Role of the cysteine residues in Arabidopsis thaliana cyclophilin CYP203 in peptidyl-prolyl cis-trans isomerase and redox-related functions. Biochem J 401: 287-297, 2007.

90. Lee J, Spector D, Godon C, Labarre J, and Toledano MB. A new antioxidant with alkyl hydroperoxide defense properties in yeast. J Biol Chem 274: 4537-4544, 1999.

91. Lee KO, Jang HH, Jung BG, Chi YH, Lee JY, Choi YO, Lee JR, Lim CO, Cho MJ, and Lee SY. Rice 1Cys-peroxiredoxin over-expressed in transgenic tobacco does not maintain dormancy but enhances antioxidant activity. FEBS Lett 486: 103-106, 2000.

92. Lee SP, Hwang YS, Kim YJ, Kwon KS, Kim HJ, Kim K, and Chae HZ. Cyclophilin a binds to peroxiredoxins and activates its peroxidase activity. J Biol Chem 276: 29826-29832, 2001.

93. Lindermayr C, Saalbach G, and Durner J. Proteomic identification of S-nitrosylated proteins in Arabidopsis. Plant Physiol 137: 921-930, 2005.

94. Lindermayr C, Saalbach G, Bahnweg G, and Durner J. Differential inhibition of Arabidopsis methionine adenosyltransferases by protein S-nitrosylation. J Biol Chem 281: 4285-4291, 2006.

95. Ma S and Bohnert HJ. Gene networks in Arabidopsis thaliana for metabolic and environmental functions. Mol Biosyst 4: 199-204, 2008.

96. Ma S, Gong Q, and Bohnert HJ. An Arabidopsis gene network based on the graphical Gaussian model. Genome Res 17: 1614-1625, 2007.

97. Maeda K, Hägglund P, Björnberg O, Winther JR, and Svensson B. Kinetic and thermodynamic properties of two barley thioredoxin h isozymes, HvTrxh1 and HvTrxh2. FEBS Lett 584: 3376-3380, 2010.

98. Manevich Y, Feinstein SI, and Fisher AB. Activation of the antioxidant enzyme 1-CYS peroxiredoxin requires glutathionylation mediated by heterodimerization with pi GST. Proc Natl Acad Sci USA 101: 3780-3785, 2004.

99. Manevich $Y$ and Fisher AB. Peroxiredoxin 6, a 1-Cys peroxiredoxin, functions in antioxidant defense and lung phospholipid metabolism. Free Radicals Biol Med 38: 14221432, 2005.

100. Manfield IW, Jen CH, Pinney JW, Michalopoulos I, Bradford JR, Gilmartin PM, and Westhead DR. Arabidopsis coexpression tool (ACT): web server tools for microarraybased gene expression analysis. Nucleic Acids Res 34: W504W509, 2006.

101. Mannick JB and Schonhoff CM. NO means no and yes: regulation of cell signalling by protein nitrosylation. Free Radic Res 38: 1-7, 2004.

102. Marty L, Siala W, Schwarzländer M, Fricker MD, Wirtz M, Sweetlove LJ, Meyer Y, Meyer AJ, Reichheld JP, and Hell R. The NADPH-dependent thioredoxin system constitutes a functional backup for cytosolic glutathione reductase in Arabidopsis. Proc Natl Acad Sci USA 106: 9109 9114, 2009.

103. Matamoros MA, Loscos J, Dietz KJ, Aparicio-Tejo PM, and Becana M. Function of antioxidant enzymes and metabolites during maturation of pea fruits. J Exp Bot 61: 87-97, 2010.

104. Meyer AJ, Brach T, Marty L, Kreye S, Rouhier N, Jacquot JP, and Hell R. Redox-sensitive GFP in Arabidopsis thaliana is a quantitative biosensor for the redox potential of the cellular glutathione redox buffer. Plant J 52: 973-986, 2007.

105. Meyer Y, Buchanan BB, Vignols F, and Reichheld JP. Thioredoxins and glutaredoxins: unifying elements in redox biology. Annu Rev Genet 43: 335-367, 2009.

106. Michalska J, Zauber H, Buchanan BB, Cejudo FJ, and Geigenberger P. NTRC links built-in thioredoxin to light and sucrose in regulating starch synthesis in chloroplasts and amyloplasts. Proc Natl Acad Sci USA 106: 9908-9913, 2009.

107. Miller G, Suzuki N, Ciftci-Yilmaz S, and Mittler R. Reactive oxygen species homeostasis and signalling during drought and salinity stresses. Plant Cell Environ 33: 453-467, 2010.

108. Mittler R, Vanderauwera S, Gollery M, and Van Breusegem F. Reactive oxygen gene network of plants. Trends Plant Sci 9: 490-498, 2004.

109. Monteiro G, Horta BB, Pimenta DC, Augusto O, and Netto LES. Reduction of 1-Cys peroxiredoxins by ascorbate changes the thiol-specific antioxidant paradigm, revealing another function of vitamin C. Proc Natl Acad Sci USA 104: 4886-4891, 2007.

110. Moon JC, Jang HH, Chae HB, Lee JR, Lee SY, Jung YJ, Shin MR, Lim HS, Chung WS, Yun DJ, Lee KO, and Lee SY. The C-type Arabidopsis thioredoxin reductase ANTR-C acts as an electron donor to 2-Cys peroxiredoxins in chloroplasts. Biochem Biophys Res Commun 348: 478-484, 2006.

111. Moreau M, Lindermayr C, Durner J, and Klessig DF. NO synthesis and signaling in plants-where do we stand? Physiol Plant 138: 372-383, 2010.

112. Motohashi K and Hisabori T. CcdA is a thylakoid membrane protein required for the transfer of reducing equivalents from stroma to thylakoid lumen in the higher plant chloroplast. Antiox Redox Signal 13: 1169-1176, 2010.

113. Motohashi $\mathrm{K}$ and Hisabori T. HCF164 receives reducing equivalents from stromal thioredoxin across the thylakoid membrane and mediates reduction of target proteins in the thylakoid lumen. J Biol Chem 281: 35039-35047, 2006.

114. Motohashi K, Kondoh A, Stumpp MT, and Hisabori T. Comprehensive survey of proteins targeted by chloroplast thioredoxin. Proc Natl Acad Sci USA 98: 11224-11229, 2001.

115. Motohashi K, Romano PGN, and Hisabori T. Identification of thioredoxin targeted proteins using thioredoxin single cysteine mutant-immobilized resin. Methods Mol Biol 479, 117-131, 2009.

116. Mowla SB, Thomson JA, Farrant JM, and Mundree SG. A novel stress-inducible antioxidant enzyme identified from the resurrection plant Xerophyta viscosa Baker. Planta 215: 716-726, 2002.

117. Müller MJ and Berger S. Reactive electrophilic oxylipins: pattern recognition and signalling. Phytochemistry 70: 15111521, 2009.

118. Muthuramalingam M, Seidel T, Laxa M, de Miranda SMN, Gärtner F, Ströher E, Kandlbinder A, and Dietz KJ. Multiple redox and non-redox interactions define 2-Cys peroxiredoxin as a regulatory hub in the chloroplast. Mol Plant 2: 1273-1288, 2009.

119. Nakagami H, Sugiyama N, Mochida K, Daudi A, Yoshida Y, Toyoda T, Tomita M, Ishihama Y, and Shirasu K. Largescale comparative phosphoproteomics identifies conserved phosphorylation sites in plants. Plant Physiol 153: 11611174, 2010. 
120. Navrot N, Collin V, Gualberto J, Gelhaye E, Hirasawa M, Rey P, Knaff DB, Issakidis E, Jacquot JP, and Rouhier N. Plant glutathione peroxidases are functional peroxiredoxins distributed in several subcellular compartments and regulated during biotic and abiotic stresses. Plant Physiol 142: 1364-1379, 2006.

121. Navrot N, Rouhier N, Gelhaye E, and Jacquot JP. Reactive oxygen species generation and antioxidant systems in plant mitochondria. Physiol Plant 129: 185-195, 2007.

122. Nelson KJ, Parsonage D, Hall A, Karplus PA, and Poole LB. Cysteine $\mathrm{pK}(\mathrm{a})$ values for the bacterial peroxiredoxin AhpC. Biochem 47: 12860-12868, 2008.

123. Noguera-Mazon V, Krimm I, Walker O, and Lancelin JM. Protein-protein interactions within peroxiredoxin systems. Photosynth Res 89: 277-290, 2006.

124. Noguera-Mazon V, Lemoine J, Walker O, Rouhier $\mathrm{N}$, Salvador A, Jacquot JP, Lancelin JM, and Krimm I. Glutathionylation induces the dissociation of 1-Cys Dperoxiredoxin non-covalent homodimer. J Biol Chem 281: 31736-31742, 2006.

125. Noh YH, Baek JY, Jeong W, Rhee SG, and Chang TS. Sulfiredoxin translocation into mitochondria plays a crucial role in reducing hyperoxidized peroxiredoxin III. J Biol Chem 284: 8470-8477, 2009.

126. Oelze ML, Kandlbinder A, and Dietz KJ. Redox regulation and overreduction control in the photosynthesizing cell: complexity in redox regulatory networks. Biochim Biophys Acta 1780: 1261-1272, 2008.

127. Parsonage D, Karplus PA, and Poole LB. Substrate specificity and redox potential of $\mathrm{AhpC}$, a bacterial peroxiredoxin. Proc Natl Acad Sci USA 105: 8209-8214, 2008.

128. Parsonage D, Youngblood DS, Sarma GN, Wood ZA, Karplus PA, and Poole LB. Analysis of the link between enzymatic activity and oligomeric state in AhpC, a bacterial peroxiredoxin. Biochemistry 44: 10583-10592, 2005.

129. Pascual MB, Mata-Cabana A, Florencio FJ, Lindahl M, and Cejudo FJ. Overoxidation of 2-Cys peroxiredoxin in prokaryotes: cyanobacterial 2-Cys peroxiredoxins sensitive to oxidative stress. J Biol Chem 285: 34485-34492, 2010.

130. Peltier JB, Cai Y, Sun Q, Zabrouskov V, Giacomelli L, Rudella A, Ytterberg AJ, Rutschow $\mathrm{H}$, and van Wijk KJ. The oligomeric stromal proteome of Arabidopsis thaliana chloroplasts. Mol Cell Proteomics 5: 114-133, 2006.

131. Pena-Ahumada A, Kahmann U, Dietz KJ, and Baier M. Regulation of peroxiredoxin expression versus expression of Halliwell-Asada-Cycle enzymes during early seedling development of Arabidopsis thaliana. Photosynth Res 89: 99112, 2006.

132. Pérez-Pérez ME, Mata-Cabana A, Sánchez-Riego AM, Lindahl M, and Florencio FJ. A comprehensive analysis of the peroxiredoxin reduction system in the cyanobacterium Synechocystis sp. strain PCC 6803 reveals that all five peroxiredoxins are thioredoxin dependent. J Bacteriol 191: 7477-7489, 2009.

133. Pérez-Ruiz JM, Spínola MC, Kirchsteiger K, Moreno J, Sahrawy M, and Cejudo FJ. Rice NTRC is a high-efficiency redox system for chloroplast protection against oxidative damage. Plant Cell 18: 2356-2368, 2006.

134. Pesaresi $\mathrm{P}$, Schneider $\mathrm{A}$, Kleine $\mathrm{T}$, and Leister D. Interorganellar communication. Curr Opin Plant Biol 10: 600606, 2007.

135. Peskin AV, Low FM, Paton LN, Maghzal GJ, Hampton MB, and Winterbourn CC. The high reactivity of peroxiredoxin
2 with $\mathrm{H}_{2} \mathrm{O}_{2}$ is not reflected in its reaction with other oxidants and thiol reagents. J Biol Chem 282: 11885-11892, 2007.

136. Peter E, Rothbart M, Oelze ML, Shalygo N, Dietz KJ, and Grimm B. Mg protoporphyrin monomethylester cyclase deficiency and effects on tetrapyrrole metabolism in different light conditions. Plant Cell Physiol 51: 1229-1241, 2010.

137. Petersson UA, Kieselbach T, García-Cerdán JG, and Schröder WP. The PrxQ protein of Arabidopsis thaliana is a member of the luminal chloroplast proteome. FEBS Lett 580: 6055-6061, 2006.

138. Pitsch NT, Witsch B, and Baier M. Comparison of the chloroplast peroxidase system in the chlorophyte Chlamydomonas reinhardtii, the bryophyte Physcomitrella patens, the lycophyte Selaginella moellendorffii and the seed plant Arabidopsis thaliana. BMC Plant Biol 10: 133, 2010.

139. Pogson BJ, Woo NS, Förster B, and Small ID. Plastid signalling to the nucleus and beyond. Trends Plant Sci 13: 602609, 2008.

140. Poole LB. The catalytic mechanism of peroxiredoxins. Subcell Biochem 44: 61-81, 2007.

141. Pukacka S and Ratajczak E. Antioxidative response of ascorbate-glutathione pathway enzymes and metabolites to desiccation of recalcitrant Acer saccharinum seeds. J Plant Physiol 163: 1259-1266, 2006.

142. Pulido P, Spínola MC, Kirchsteiger K, Guinea M, Pascual MB, Sahrawy M, Sandalio LM, Dietz KJ, González M, and Cejudo FJ. Functional analysis of the pathways for 2-Cys peroxiredoxin reduction in Arabidopsis thaliana chloroplasts. J Exp Bot 61: 4043-4054, 2010.

143. Ralat LA, Manevich Y, Fisher AB, and Colman RF. Direct evidence for the formation of a complex between 1-cysteine peroxiredoxin and glutathione S-transferase pi with activity changes in both enzymes. Biochemistry 45: 360-372, 2006.

144. Reichert A, Baalmann E, Vetter S, Backhausen JE, and Scheibe R. Activation properties of the redox-modulated chloroplast enzymes glyceraldehyde 3-phosphate dehydrogenase and fructose-1,6-bisphosphatase. Physiol Plant 110: 330-341, 2000.

145. Reiland S, Messerli G, Baerenfaller K, Gerrits B, Endler A, Grossmann J, Gruissem W, and Baginsky S. Large-scale Arabidopsis phosphoproteome profiling reveals novel chloroplast kinase substrates and phosphorylation networks. Plant Physiol 150: 889-903, 2009.

146. Rey P, Cuiné S, Eymery F, Garin J, Court M, Jacquot JP, Rouhier N, and Broin M. Analysis of the proteins targeted by CDSP32, a plastidic thioredoxin participating in oxidative stress responses. Plant J 41: 31-42, 2005.

147. Romero-Puertas MC, Campostrini N, Mattè A, Righetti PG, Perazzolli M, Zolla L, Roepstorff P, and Delledonne M. Proteomic analysis of S-nitrosylated proteins in Arabidopsis thaliana undergoing hypersensitive response. Proteomics 8: 1459-1469, 2008.

148. Romero-Puertas MC, Laxa M, Matte A, Zaninotto F, Finkemeier I, Jones AME, Perazzolli M, Vandelle E, Dietz KJ, and Delledonne M. S-nitrosylation of peroxiredoxin II E promotes peroxynitrite-mediated tyrosine nitration. Plant Cell 19: 4120-4130, 2007.

149. Rouhier N, Gelhaye E, Gualberto JM, Jordy MN, De Fay E, Hirasawa M, Duplessis S, Lemaire SD, Frey P, Martin F, Manieri W, Knaff DB, and Jacquot JP. Poplar peroxiredoxin Q. A thioredoxin-linked chloroplast antioxidant functional in pathogen defense. Plant Physiol 134: 10271038, 2004. 
150. Rouhier N, Gelhaye E, and Jacquot JP. Glutaredoxindependent peroxiredoxin from poplar: protein-protein interaction and catalytic mechanism. J Biol Chem 277: 13609-13614, 2002.

151. Rouhier N, Gelhaye E, Sautiere PE, Brun A, Laurent P, Tagu D, Gerard J, de Faÿ E, Meyer Y, and Jacquot JP. Isolation and characterization of a new peroxiredoxin from poplar sieve tubes that uses either glutaredoxin or thioredoxin as a proton donor. Plant Physiol 127: 1299-1309, 2001

152. Rouhier N and Jacquot JP. The plant multigenic family of thiol peroxidases. Free Radic Biol Med 38: 1413-1421, 2005.

153. Rouhier N, Villarejo A, Srivastava M, Gelhaye E, Keech O, Droux M, Finkemeier I, Samuelsson G, Dietz KJ, Jacquot JP, and Wingsle G. Identification of plant glutaredoxin targets. Antioxid Redox Signal 7: 919-929, 2005.

154. Rouhier N. Plant glutaredoxins: pivotal players in redox biology and iron-sulfur centre assembly. New Phytol 186: 365-372, 2010.

155. Sakamoto A, Tsukamoto $S$, Yamamoto H, Ueda-Hashimoto M, Takahashi M, Suzuki H, and Morikawa H. Functional complementation in yeast reveals a protective role of chloroplast 2-Cys peroxiredoxin against reactive nitrogen species. Plant J 33: 841-851, 2003.

156. Sayed AA and Williams DL. Biochemical characterization of 2-Cys peroxiredoxins from Schistosoma mansoni. J Biol Chem 279: 26159-26166, 2004.

157. Schubert M, Petersson UA, Haas BJ, Funk C, Schröder WP, and Kieselbach T. Proteome map of the chloroplast lumen of Arabidopsis thaliana. J Biol Chem 277: 8354-8365, 2002.

158. Schürmann $\mathrm{P}$ and Buchanan BB. The ferredoxin/thioredoxin system of oxygenic photosynthesis. Antiox Redox Signal 10: 1235-1274, 2008.

159. Seidel T, Seefeldt B, Sauer M, and Dietz KJ. In vivo-analysis of the 2-Cys peroxiredoxin oligomeric state by two-step FRET. J Biotech 149: 272-279, 2010.

160. Seo JH, Lim JC, Lee DY, Kim KS, Piszczek G, Nam HW, Kim YS, Ahn T, Yun CH, Kim K, Chock PB, and Chae HZ. Novel protective mechanism against irreversible hyperoxidation of peroxiredoxin: N-alpha-terminal acetylation of human peroxiredoxin II. J Biol Chem 284: 13455-13465, 2009.

161. Shaikhali J, Heiber I, Seidel T, Ströher E, Hiltscher H, Birkmann S, Dietz KJ, and Baier M. The redox-sensitive transcription factor Rap2.4a controls nuclear expression of 2-Cys peroxiredoxin $\mathrm{A}$ and other chloroplast antioxidant enzymes. BMC Plant Biol 8: 48, 2008.

162. Shlomai J. Redox control of protein-DNA interactions: from molecular mechanisms to significance in signal transduction, gene expression, and DNA replication. Antioxid Redox Signal 13: 1429-1476, 2010.

163. Sideri TC, Stojanovski K, Tuite MF, and Grant CM. Ribosome-associated peroxiredoxins suppress oxidative stressinduced de novo formation of the [PSI+] prion in yeast. Proc Natl Acad Sci USA 107: 6394-6399, 2010.

164. Sischka A, Spiering A, Khaksar M, Laxa M, Konig J, Dietz KJ, and Anselmetti D. Dynamic translocation of ligand-complexed DNA through solid-state nanopores with optical tweezers. J Physics-Condensed Matter 22: 454121, 2010.

165. Spínola MC, Pérez-Ruiz JM, Pulido P, Kirchsteiger $K$, Guinea M, González M, and Cejudo FJ. NTRC: new ways of using NADPH in the chloroplast. Physiol Plant 133: 516524, 2008.

166. Stacy RA, Munthe E, Steinum T, Sharma B, and Aalen RB. A peroxiredoxin antioxidant is encoded by a dormancyrelated gene, Per1, expressed during late development in the aleurone and embryo of barley grains. Plant Mol Biol 31: 1205-1216, 1996.

167. Stacy RA, Nordeng TW, Culiáñez-Macià FA, and Aalen RB. The dormancy-related peroxiredoxin anti-oxidant, PER1, is localized to the nucleus of barley embryo and aleurone cells. Plant J 19: 1-8, 1999.

168. Stenbaek A, Hansson A, Wulff RP, Hansson M, Dietz KJ, and Jensen PE. NADPH-dependent thioredoxin reductase and 2-Cys peroxiredoxins are needed for the protection of Mg-protoporphyrin monomethyl ester cyclase. FEBS Lett 582: 2773-2778, 2008.

169. Stork T, Laxa M, Dietz MS, and Dietz KJ. Functional characterisation of the peroxiredoxin gene family members of Synechococcus elongatus PCC 7942. Arch Microbiol 191: 141-151, 2009.

170. Stork T, Michel KP, Pistorius EK, and Dietz KJ. Bioinformatic analysis of the genomes of the cyanobacteria Synechocystis sp. PCC 6803 and Synechococcus elongatus PCC 7942 for the presence of peroxiredoxins and their transcript regulation under stress. J Exp Bot 56: 3193-3206, 2005.

171. Ströher E and Dietz KJ. Concepts and approaches towards understanding the cellular redox proteome. Plant Biol 8: 407-418, 2006.

172. Sweetlove LJ, Heazlewood JL, Herald V, Holtzapffel R, Day DA, Leaver CJ, and Millar AH. The impact of oxidative stress on Arabidopsis mitochondria. Plant J 32: 891904, 2002.

173. Tichy $\mathrm{M}$ and Vermaas W. In vivo role of catalase-peroxidase in Synechocystis sp. strain PCC 6803. J Bacteriol 181: 18751882, 1999.

174. Torres MA. ROS in biotic interactions. Physiol Plant 138: 414-429, 2010.

175. Trujillo M, Clippe A, Manta B, Ferrer-Sueta G, Smeets A, Declercq JP, Knoops B, and Radi R. Pre-steady state kinetic characterization of human peroxiredoxin 5: Taking advantage of Trp84 fluorescence increase upon oxidation. Arch Biochem Biophys 467: 95-106, 2007.

176. Trujillo M, Ferrer-Sueta G, Thomson L, Flohé L, and Radi R. Kinetics of peroxiredoxins and their role in the decomposition of peroxynitrite. Subcell Biochem 44: 83-113, 2007.

177. Veal EA, Findlay VJ, Day AM, Bozonet SM, Evans JM, Quinn J, and Morgan BA. A 2-Cys peroxiredoxin regulates peroxide-induced oxidation and activation of a stressactivated MAP kinase. Mol Cell 15: 129-139, 2004

178. Verdoucq L, Vignols F, Jacquot JP, Chartier Y, and Meyer $\mathrm{Y}$. In vivo characterization of a thioredoxin $\mathrm{h}$ target protein defines a new peroxiredoxin family. J Biol Chem 274: 1971419722, 1999.

179. Vivancos AP, Castillo EA, Biteau B, Nicot C, Ayté J, Toledano MB, and Hidalgo E. A cysteine-sulfinic acid in peroxiredoxin regulates $\mathrm{H}_{2} \mathrm{O}_{2}$-sensing by the antioxidant Pap1 pathway. Proc Natl Acad Sci USA 102: 8875-8880, 2005.

180. Wawer I, Bucholc M, Astier J, Anielska-Mazur A, Dahan J, Kulik A, Wysłouch-Cieszynska A, Zareba-Kozioł M, Krzywinska E, Dadlez M, Dobrowolska G, and Wendehenne D. Regulation of Nicotiana tabacum osmotic stressactivated protein kinase and its cellular partner GAPDH by nitric oxide in response to salinity. Biochem J 429: 73-83, 2010. 
181. Woo HA, Yim SH, Shin DH, Kang D, Yu DY, and Rhee SG. Inactivation of peroxiredoxin I by phosphorylation allows localized $\mathrm{H}_{2} \mathrm{O}_{2}$ accumulation for cell signalling. Cell 140: 517-528, 2010

182. Wood ZA, Poole LB, and Karplus PA. Peroxiredoxin evolution and the regulation of hydrogen peroxide signalling. Science 300: 650-653, 2003.

183. Wood ZA, Schroder E, Harris JR, and Poole LB. Structure, mechanism and regulation of peroxiredoxins. Trends Biochem Sci 28: 32-40, 2003.

184. Wu Y, Feinstein SI, Manevich Y, Chowdhury I, Pak JH, Kazi A, Dodia C, Speicher DW, and Fisher AB. Mitogenactivated protein kinase-mediated phosphorylation of peroxiredoxin 6 regulates its phospholipase A(2) activity. Biochem J 419: 669-679, 2009.

185. Yamamoto H, Miyake C, Dietz KJ, Tomizawa K, Murata N, and Yokota A. Thioredoxin peroxidase in the cyanobacterium Synechocystis sp. PCC 6803. FEBS Lett 447: 269-273, 1999.

186. Yamazaki D, Motohashi K, Kasama T, Hara Y, and Hisabori T. Target proteins of the cytosolic thioredoxins in Arabidopsis thaliana. Plant Cell Physiol 45: 18-27, 2004.

Address correspondence to:

Prof. Karl-Josef Dietz

Department of Biochemistry and Physiology of Plants

Bielefeld University

University St. 25

Bielefeld 33501

Germany

E-mail: karl-josef.dietz@uni-bielefeld.de

Date of first submission to ARS Central, September 25, 2010; date of final revised submission, December 15, 2010; date of acceptance, January 1, 2011.

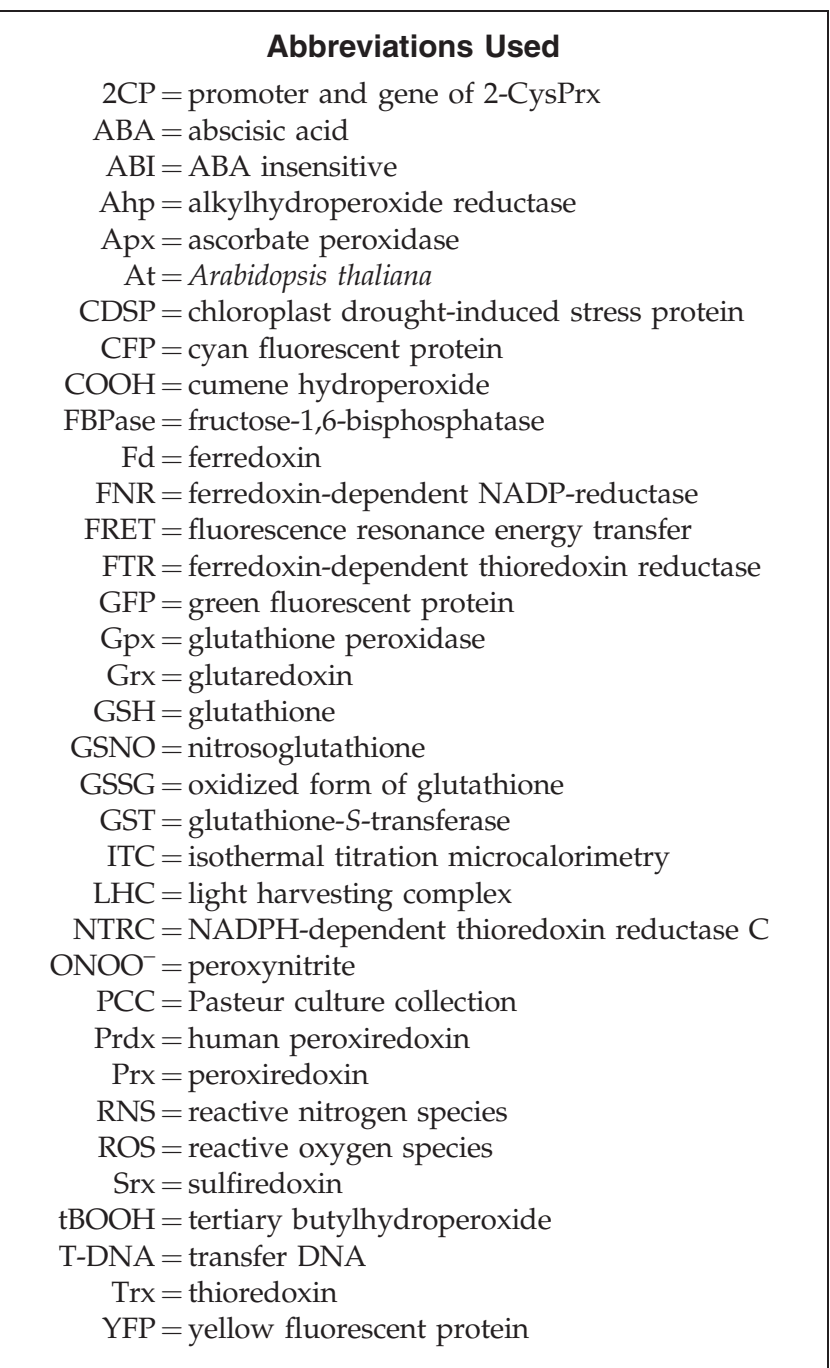


